MPP-2006-5

to appear in Commun. Math. Phys.

\title{
Structure of the space of ground states in systems with non-amenable symmetries
}

\author{
M. Niedermaier* and E. Seiler \\ Laboratoire de Mathematiques et Physique Theorique \\ CNRS/UMR 6083, Université de Tours \\ Parc de Grandmont, 37200 Tours, France \\ Max-Planck-Institut für Physik \\ Föhringer Ring 6 \\ 80805 München, Germany
}

\begin{abstract}
We investigate classical spin systems in $d \geq 1$ dimensions whose transfer operator commutes with the action of a nonamenable unitary representation of a symmetry group, here $\mathrm{SO}(1, N)$; these systems may alternatively be interpreted as systems of interacting quantum mechanical particles moving on hyperbolic spaces. In sharp contrast to the analogous situation with a compact symmetry group the following results are found and proven: (i) Spontaneous symmetry breaking already takes place for finite spatial volume/finitely many particles and even in dimensions $d=1,2$. The tuning of a coupling/temperature parameter cannot prevent the symmetry breaking. (ii) The systems have infinitely many non-invariant and non-normalizable generalized ground states. (iii) the linear space spanned by these ground states carries a distinguished unitary representation of $\mathrm{SO}(1, N)$, the limit of the spherical principal series. (iv) The properties (i)-(iii) hold universally, irrespective of the details of the interaction.
\end{abstract}

${ }^{*}$ Membre du CNRS; e-mail: max@phys.univ-tours.fr 


\section{Contents}

\begin{tabular}{lll}
\hline 1 & Introduction & 2
\end{tabular}

2 Group decomposition of the state space 4

$2.1 \quad$ Generalized spin svstems $\ldots \ldots \ldots \ldots$

2.2 The spectral problem . . . . . . . . . . . . . . . . . . . . . . . . . . . 10

2.3 Orbit decomposition . . . . . . . . . . . . . . . . . . . . . 13

2.4 The reduction of $\rho(G)$ on $L^{2}\left(\mathcal{M}_{n}\right) \ldots \ldots \ldots \ldots \ldots$

$3 \quad$ The spectral problem on the invariant fibers 26

3.1 Basic consequences of the $\rho(G)$ invariance $\ldots \ldots \ldots \ldots$

3.2 Fiber decomposition of invariant selfadioint operators . . . . . . . . . . . 29

3.3 Relating the spectral problems of $\mathbf{A}$ and $\mathbf{A}_{\kappa}: \mathbf{A}_{\kappa}$ compact . . . . . 33

3.4 Relating the spectral problems of $\mathbf{A}$ and $\mathbf{A}_{\tau}$ : $\mathbf{A}_{\sigma}$ not necessarily compact 40

3.5 Absence of the discrete series $\ldots \ldots \ldots \ldots \ldots$

\begin{tabular}{|lll}
4 & The structure of the ground state sector & 47
\end{tabular}

$4.1 \mathcal{G}_{\sigma}(\mathbf{T})$ is empty for all but one principal series representation $\ldots . .47$

4.2 The role of strict positivity $\ldots \ldots \ldots \ldots \ldots$. . . . . . . . . . 51

$4.3 \mathcal{G}_{\sigma}(\mathbf{T})$ for $\mathbf{T}_{\sigma}$ compact $\ldots \ldots \ldots \ldots \ldots \ldots \ldots \ldots$

5 Conclusions and outlook 54

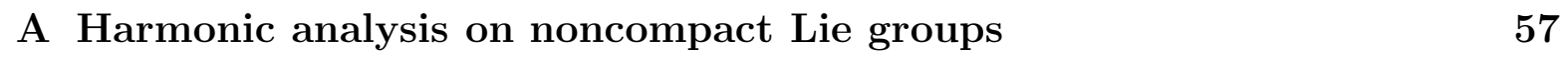

\begin{tabular}{|ll}
\hline B The restricted dual of $\mathrm{SO}(1, \mathrm{~N})$ & 73
\end{tabular}

\begin{tabular}{ll}
\hline C The amenable case ISO(N) & 80
\end{tabular} 


\section{Introduction}

Spontaneous symmetry breaking is typically discussed for compact internal or for abelian translational symmetries, see e.g. [45, 48, 37]. Both share the property of being amenable 42 and their spontaneous breakdown is a specific dynamical property of the interaction. Here we consider systems with a nonamenable symmetry, by which we mean that the dynamics is invariant under a nonamenable unitary representation [4] of a locally compact group (which then by necessity is also nonamenable). One goal of this note is to show, roughly, that whenever the dynamics of a system of classical statistical mechanics is invariant under a nonamenable symmetry, this symmetry is always spontaneously broken, irrespective of the details of the interaction. Neither does the long or short ranged nature of the interaction matter, nor can the tuning of a (temperature) parameter prevent the symmetry breaking. Spontaneous symmetry breaking even occurs in one and two dimensions, where for compact symmetries this is ruled out by the Mermin-Wagner theorem. The phenomenon is not limited to a semiclassical regime and occurs already for systems with finitely many degrees of freedom.

The systems will be defined on a finite lattice $\Lambda$ of arbitrary dimension and connectivity. The dynamical variables are 'spins' attached to the vertices of the lattice, taking values in some noncompact Riemannian symmetric space $Q=G / K$, where $G$ is the noncompact symmetry group and $K$ a maximally compact subgroup. The dynamics is specified by a transfer operator acting on the square integrable functions on the configuration manifold $Q^{\Lambda}$. Such a system can alternatively be interpreted as a quantum mechanical system of finitely many particles living on $Q$; we only have to interpret the transfer matrix as $\exp (-H)$ and thereby define the Hamiltonian $H$; the inevitable spontaneous symmetry breaking appears then as degeneracy of the generalized ground states of this system. Conversely, given a quantum mechanical system with a Hamiltonian $H$, we can re-interpret the system as one of classical statistical mechanics with $\exp (-H)$ as the transfer matrix. To fix ideas one may take a Hamiltonian of the conventional form

$$
H=-\frac{1}{2} \sum_{i=1}^{\nu} \Delta_{i}+\sum_{i, k=1}^{\nu} V_{i k}
$$

where $\Delta_{i}$ is the Laplace-Beltrami operator on $Q$ for the i-th particle and the $V_{i k}$ are some potentials describing the interaction of particle $i$ and $k$, depending only on the geodesic distances of the particles. Typically one would also require that the set $\Lambda=\{1,2 \ldots \nu\}$ has the structure of a lattice and that the interaction links neighboring sites only. The Hamiltonians (1.1) are however only one class of examples, many others are covered. Indeed apart from some technical conditions on the transfer matrix it is mostly the invariance that matters.

To analyze these systems, it is necessary to perform something analogous to the wellknown separation of the center of mass motion from the relative motion in Euclidean 
space. This turns out to be considerably more involved in our setting. It is convenient not to define an actual center of mass of the $\nu$ spins or particles, respectively, but rather take simply one of them as parameterizing the global position of the configuration. The universality of the resulting symmetry breaking is then related to the fact that the global motion of these systems never allows for a symmetric (proper or generalized) ground state.

Normally spontaneous symmetry breaking can only happen in the thermodynamic limit. We stress again that here the situation is different: spontaneous symmetry breaking takes place already on a finite spatial lattice. The systems exhibit a remarkable universality in the structure of their generalized ground states. Namely, there are always infinitely many non-normalizable and non-invariant ground states which transform irreducibly under a preferred representation of the group - the same for a large class of transfer operators! All generalized ground states can be generated by forming linear combinations of factorized wave functions, where one factor describes the global and the other one the relative motion; of course the second factor will be sensitive to the interaction as far as the relative motion is concerned; what is universal is the transformation law under global symmetry transformations of the first factor.

In quantum one-particle systems described by an exactly soluble Schrödinger equation an infinite degeneracy in the ground state energy has been found earlier: first of all in the well known problem of the Landau levels in the Euclidean plane; closer to our situation explicitly in [8] for the supersymmetric $\mathrm{SO}(1,2)$ invariant quantum mechanics and implicitly in 31] (p.172), 61] and in [7] for the lowest Landau level. The interplay between spontaneous symmetry breaking, nonamenability and properties of the transfer operator was understood in [38], initially for the hyperbolic spin chain.

The thermodynamic limit can usually only be taken on the level of correlation functions, so that the 'fate' of the ground state orbit cannot directly be traced. By means of an Osterwalder-Schrader reconstruction one can in principle recover a Hilbert space description, however inevitably one with an exotic structure; cf. 38].

Our main example for $Q$ will be the hyperboloids $\mathbb{H}_{N}:=\mathrm{SO}_{0}(1, N) / \mathrm{SO}(N), N \geq 2$, in part because of the importance of the Lorentz and de Sitter groups in physics, and in part because already for the groups $\mathrm{SO}_{0}(1, N)$ the harmonic analysis exhibits all of the characteristic complications. Most of the constructions however generalize to a large class of noncompact coset spaces and are actually easier to understand in a general setting. We thus specialize to $Q=\mathbb{H}_{N}$ only when needed.

Let us now make things a little more explicit: the configuration space $\mathcal{M}$ is the direct product of $\nu:=|\Lambda|$ copies of the space $Q$. A hypercubical lattice $\Lambda \subset \mathbb{Z}^{d}$ of arbitrary dimension $d$ is a prime example, however neither the dimension nor the connectivity of the lattice is essential. The pure states of the system are described by elements of $L^{2}(\mathcal{M})$, i.e. functions $\psi: \mathcal{M} \rightarrow \mathbb{C}$, square integrable with respect to the invariant measure $d \gamma$. The left diagonal $G$ action $d$ on $\mathcal{M}$ induces a unitary representation $\ell_{\mathcal{M}}$ of $G$ on $L^{2}(\mathcal{M})$ via $\left(\ell_{\mathcal{M}}(g) \psi\right)(m)=\psi\left(g^{-1} m\right)$. Since $L^{2}(\mathcal{M}) \simeq\left[L^{2}(Q)\right]^{\otimes \nu}$ it can be 
identified with the $\nu$-fold inner tensor product of the left quasiregular representation $\ell_{1}$. On this $L^{2}$ space we consider bounded selfadjoint operators $\mathbf{A}$ commuting with the group action, i.e. $\ell_{\mathcal{M}} \circ \mathbf{A}=\mathbf{A} \circ \ell_{\mathcal{M}}$. Such operators $\mathbf{A}$ can only have essential spectrum.

Specifically we consider so-called transfer operators defining the dynamics of the system. A precise definition is given in Definition 2.1 below. Here it may suffice to say that a transfer operator is a bounded selfadjoint operator on $L^{2}$ which is positive as well as positivity improving; as usual the latter property is realized by taking for $\mathbf{T}$ an integral operator with strictly positive kernel, $T\left(m, m^{\prime}\right)>0$ for all $m, m^{\prime} \in \mathcal{M}$. We are interested in an invariant dynamics, so we assume $\ell_{\mathcal{M}} \circ \mathbf{T}=\mathbf{T} \circ \ell_{\mathcal{M}}$. Important examples are $\mathbf{T}=$ $\exp (-H)$, with $H$ as in (1.1), but Hamiltonians with more complicated 'time derivative' terms and non-pair potentials would also be allowed. The latter is welcome because such complicated Hamiltonians naturally arise as the result of blocking transformations. It is easy to see that an invariant transfer operator $\mathbf{T}$ cannot be a compact operator, furthermore it cannot even have normalizable ground states, i.e. solutions of $\mathbf{T} \psi=\|\mathbf{T}\| \psi$, with $\psi \in L^{2}$. (In fact $\|\mathbf{T}\|$ must either lie in the continuous spectrum of $\mathbf{T}$ or be a limit of eigenvalues with infinite multiplicity). Instead our setting is such that $\mathbf{T}$ has a continuous extension to an operator from $L^{p}$ to $L^{p}$ for $1 \leq p \leq \infty$ and we identify conditions under which $\mathbf{T}$ has generalized ground states. These we take as almost everywhere defined functions (not just distributions) which are eigenfunctions with spectral value $\|\mathbf{T}\|$. The set of these generalized ground states forms a linear space which we call the ground state sector $\mathcal{G}(\mathbf{T})$ of $\mathbf{T}$. It is important that $\mathbf{T}$ is defined as a selfadjoint operator on a Hilbert space, here $L^{2}(\mathcal{M})$, so that the spectral theorem can be applied to provide a resolution of the identity. The notion of a generalized ground state is then unambiguous, although there is some freedom in the choice of the topological vector space in which the ground state wave functions live.

The separation of global and relative configurations is achieved by writing $\mathcal{M}=Q \times \mathcal{N}$, where $\mathcal{N}$ collects the 'relative degrees of freedom'; the construction is done in such a way that $\mathcal{M}=(G \times \mathcal{N}) / d(K)$, where $d(K)$ is the right diagonal action of $K$ on $G \times \mathcal{N}$. The details of this construction will be given in Section 2. The global part of the configurations can now be Fourier transformed: the $L^{2}$ functions on $G$ have a Plancherel-type decomposition $\int^{\oplus} d \nu(\sigma) \mathcal{L}_{\sigma} \otimes \check{\mathcal{L}}_{\breve{\sigma}}$, where the fiber spaces $\mathcal{L}_{\sigma}$ carry the unitary (infinite dimensional) $\nu$-almost always irreducible representations $\pi_{\sigma}$, and $\nu$ is carried by the so-called restricted dual $\widehat{G}_{r}$ of $G$. We show in Section 3 that one can associate to an invariant selfadjoint operator $\mathbf{A}$ on $L^{2}(\mathcal{M})$ a $\nu$-measurable field of bounded selfadjoint operators $\mathbf{A}_{\sigma}$ on $L^{2}(\mathcal{N}) \otimes \mathcal{L}_{\sigma}, \sigma \in \widehat{G}_{r}$, via

$$
\mathbf{A}=\int d \nu(\sigma)\left(\mathbb{I} \otimes \check{\mathbf{A}}_{\check{\sigma}}\right), \quad L^{2}(\mathcal{M})=\int d \nu(\sigma) \mathcal{L}_{\sigma}^{2}(\mathcal{M})
$$

In the second formula we indicated that the state space $L^{2}(\mathcal{M})$ decomposes into fibers which carry the representation $\pi_{\sigma}$ and which are preserved under the action of $\mathbb{1} \otimes \check{\mathbf{A}}_{\check{\sigma}}$. Each of the fiber spaces is isometric to $\mathcal{L}_{\sigma}^{2}(\mathcal{M}) \cong \mathcal{L}_{\sigma} \otimes \check{\mathcal{L}}_{\breve{\sigma}} \otimes L^{2}(\mathcal{N})$; however its elements 
will be realized as functions on $\mathcal{M}$. This is done by constructing for $v \in \mathcal{L}_{\sigma}$ an (antilinear) map $\tau_{v \sigma}: L^{2}(\mathcal{N}) \otimes \mathcal{L}_{\sigma} \rightarrow \mathcal{L}_{\sigma}^{2}(\mathcal{M}), f \mapsto \tau_{v \sigma}(f)$. Roughly, the image function arises by reinterpreting the matrix element $\left(f(n), \pi_{\sigma}(g) v\right)_{\sigma}$ as a (generically not square integrable) function on $\mathcal{M}$. The map is designed such that it is an isometry onto its image and has the following intertwining properties

$$
\begin{aligned}
\tau_{v \sigma}(f)\left(g^{-1} m\right) & =\tau_{\pi_{\sigma}(g) v, \sigma}(f)(m), \\
{\left[\mathbf{A} \tau_{v \sigma}(f)\right](m) } & =\tau_{v \sigma}\left(\mathbf{A}_{\sigma} f\right)(m) .
\end{aligned}
$$

According to the first equation the $G$-action on the argument of the function just rotates the reference vector $v \in \mathcal{L}_{\sigma}$ with the representation $\pi_{\sigma}$. In the second relation we anticipated that the action of $\mathbf{A}$ can be extended to the (in general non- $L^{2}$ ) functions $\tau_{v \sigma}(f)$. In view of (1.3) it is plausible that the spectral problems of $\mathbf{A}$ and $\mathbf{A}_{\sigma}$ are related as follows: suppose first that the eigenvalue equations $\mathbf{A}_{\sigma} \chi=\lambda \chi$ and $\mathbf{A} \Omega=\lambda \Omega$ are well-defined, with $\lambda \in \operatorname{Spec}(\mathbf{A})$ and (generically non- $L^{2}$ ) eigenfunctions $\chi, \Omega$, and that second the map $\tau_{v \sigma}$ admits an extension to the generalized eigenfunctions $\chi$ of $\mathbf{A}_{\sigma}$. Then by (1.3b) the image function $\tau_{v \sigma}(\chi)$ should be an eigenfunction of $\mathbf{A}$ enjoying the equivariance property (1.3).

This construction principle can be implemented for a large class of invariant selfadjoint operators A specified in Sections 3.3 and 3.4. In overview the result is that a set of generalized eigenfunctions $\Omega_{\lambda \sigma}$ exists for almost all $\lambda \in \operatorname{Spec}(\mathbf{A})$ and $\sigma \in \widehat{G}_{r}$ with the following properties: (i) they are almost everywhere defined functions (not distributions) $\Omega_{\lambda, \sigma}: \mathcal{M} \rightarrow \mathbb{C}$. (ii) they are $\sigma$-equivariant, i.e. they lie in the image of the maps $\tau_{e_{i} \sigma}$, where $\left\{e_{i}\right\}$ is an orthonormal basis of $\mathcal{L}_{\sigma}$ and where the domain is the linear hull of a complete set of eigenfunctions of $\mathbf{A}_{\sigma}$ with spectral value $\lambda \in \operatorname{Spec} \mathbf{A}_{\sigma}$. (iii) $\Omega_{\lambda \sigma}(g m) \rightarrow 0$, as $g$ leaves compact subsets of $G$. (iv) As $\lambda$ runs through $\operatorname{Spec}(\mathbf{A})$ and $\sigma$ runs through $\widehat{G}_{r}$, the eigenfunctions $\Omega_{\lambda \sigma}$ are complete, in the sense that any smooth function can be expanded in terms of the $\Omega_{\lambda \sigma}$ and that a Parseval relation holds on $\left(L^{1} \cap L^{2}\right)(\mathcal{M})$.

The transfer operators $\mathbf{T}$ considered are special cases of such invariant selfadjoint operators, which in addition are positive and positivity improving. The spectral value relevant for the ground states of a transfer operator $\mathbf{T}$ is $\lambda=\|\mathbf{T}\|$. The important "almost all $\lambda \in \operatorname{Spec}(\mathbf{T})$ " clause in the above completeness result prevents one from getting all the generalized ground states simply by specialization. However, whenever for some $\sigma \in \widehat{G}_{r}$ a complete set of eigenfunctions of $\mathbf{T}_{\sigma}$ with spectral value $\|\mathbf{T}\|$ can be found, their images under $\tau_{e_{i} \sigma}$ will produce $\mathcal{G}_{\sigma}(\mathbf{T})$, the space of $\sigma$-equivariant generalized ground states.

Off hand of course every $\sigma \in \widehat{G}_{r}$ could occur as a "representation carried by the ground state sector" in $\mathcal{G}_{\sigma}(\mathbf{T})$. Remarkably this is not the case: under fairly broad conditions only one representation occurs and always the same! For definiteness we formulate the following results for $G=\mathrm{SO}_{0}(1, N), K=\mathrm{SO}(N), M=\mathrm{SO}(N-1)$; many aspects however are valid for any noncompact linear reductive Lie group.

Theorem 1.1. Let $\mathbf{T}$ be a transfer operator on $L^{2}(\mathcal{M}, d \gamma)$ commuting with the unitary 
representation induced by a proper action $d$ of $G$. In terms of the fiber decomposition (1.2) one has:

(a) $\mathcal{G}_{\sigma}(\mathbf{T})$ is empty for all but the principal series representations whenever one of the following holds: (i) $\mathcal{G}_{\sigma}(\mathbf{T})$ contains a strictly positive function. (ii) $\mathcal{G}_{\sigma}(\mathbf{T})$ contains a K-singlet. (iii) $\mathbf{T}_{\sigma}$ is compact.

(b) $\mathcal{G}_{\sigma}(\mathbf{T})$ is non-empty for at most one of the principal series representations - the limit of the spherical ( $M$-singlet) principal series.

Combined (a) and (b) imply that if there are generalized ground states which transform equivariantly according to some unitary irreducible representation $\pi_{\sigma}$, this representation must be - under any of the conditions (i)-(iii) and possibly others - the limit of the spherical principal series, for which we write $\pi_{00}$.

It remains to establish the existence of such generalized ground states. The known construction principles for generalized eigenfunctions (the classic ones [17, 34], as well as the one described above) are not sufficient to assure the existence of generalized ground states (neither as functions nor as distributions) - so any of the fiber spaces in Theorem 1.1 could be empty, including $\mathcal{G}_{00}(\mathbf{T})$. In a follow-up paper 39] we describe a construction principle which ensures the existence of generalized ground states in various situations; a preview is given in the conclusions. There is a simple case that is, however, important for applications, in which the existence of generalized ground states is immediate: the case that all the fiber operators $\mathbf{T}_{\sigma}$ are compact. The eigenspaces with eigenvalue $\left\|\mathbf{T}_{\sigma}\right\|$ can in principle be constructed via the well-known projector s-lim $\lim _{t \rightarrow \infty}\left(\mathbf{T}_{\sigma} /\left\|\mathbf{T}_{\sigma}\right\|\right)^{t}$ (where the limit exists in the strong operator sense). One then has the following concrete variant of Theorem 1.1:

Theorem 1.2. Let $\mathbf{T}$ be as in Theorem 1.1 and assume in addition that the fiber operators $\mathbf{T}_{\sigma}, \sigma \in \widehat{G}_{r}$ are compact. Then all fiber spaces except $\mathcal{G}_{00}(\mathbf{T})$ are empty. Further $\mathbf{T}_{00}$ is itself a transfer operator which has a unique ground state in $\mathcal{G}_{00}(\mathbf{T}) \subset L^{2}(\mathcal{N}) \otimes \mathcal{L}_{\sigma=00}$. In the realization of $\mathcal{L}_{\sigma=00}$ as $L^{2}\left(S^{N-1}\right)$ this ground state can be represented by a unique a.e. positive function $\psi_{0} \cdot \mathcal{G}(\mathbf{T})$ is the linear span of functions of the form

$$
\Omega(q, n)=\int_{S^{N-1}} d S(p) \frac{\psi_{0}(n, \vec{p})}{\left(q_{0}-\vec{q} \cdot \vec{p}\right)^{\frac{N-1}{2}}}
$$

where $q=g q^{\uparrow}=\left(q_{0}, \vec{q}\right)$. Here $\mathcal{M}$ was identified with $Q \times \mathcal{N}$ in a way that replaces the original diagonal left $G$ action by $q \mapsto g q$.

For comparison we mention here the corresponding results for amenable (compact or abelian) Lie groups. When $\mathbf{T}$ is invariant under the action of a compact Lie group, the very same setting entails that the ground states have three concordant properties: normalizability, uniqueness, and invariance. That is, there exists a normalizable, 
nondegenerate ground state, which is a group singlet. This ground state can be obtained by acting with a projector $P$ on an arbitrary $L^{2}$ function, where $P$ is obtained as the strong operator limit of the iterated (compact or trace class) transfer operator: $P=s-\lim _{t \rightarrow \infty}(\mathbf{T} /\|\mathbf{T}\|)^{t}$. When $\mathbf{T}$ does not have normalizable ground states this limit does not exist; the corresponding weak limit will be the null vector. A construction of generalized ground states based on a similar but more subtle fixed point principle will be discussed in [39]; see the conclusions for a preview.

When $\mathbf{T}$ is invariant under a noncompact amenable Lie group, for instance in the Euclidean case $Q=\operatorname{ISO}(N) / \mathrm{SO}(N)$, our construction resembles the well-known procedure of separating the center-of-mass motion (see for instance [44]). It yields a ground state sector carrying the trivial representation of $\operatorname{ISO}(N)$ and for which the center-of-mass wave function is unique (the properties of the 'internal' ground state sector replacing $\mathcal{G}\left(\mathbf{T}_{00}\right)$ again depend on the details of the interaction). Of the three concordant properties above only the normalizability is lost. In appendix $\mathrm{C}$ we specialize our constructions to this degenerate situation, to contrast it with the non-amenable case.

Both amenable cases have in common that the representation carried by the ground state sector is uniquely determined and always the same, namely the trivial one. The above results show which aspects of this picture generalize to the case of non-amenable symmetries (namely, the uniqueness of the representation and its universality) and which do not (viz, the uniqueness of the ground state). In view of Theorems 1.1 and 1.2 the limit of the spherical principal series appears to be the natural generalization of the singlet in the noncompact setting.

The article is organized as follows. In Section 2 we describe the setting in more detail and explain why the Gel'fand-Maurin theory is insufficient to account for the results we are aiming at. The fiber decomposition of $L^{2}(\mathcal{M})$ is introduced. Section 3 provides the adapted fiber decomposition of a wider class of selfadjoint operators $\mathbf{A}$ and relates the spectral problem of $\mathbf{A}$ to that of the fiber operators $\mathbf{A}_{\sigma}$. These results are then applied in Section 4 to the ground state sector of transfer operators, giving a proof of Theorem 1.1 and ramifications of it. Appendices A and B provide the necessary background on the harmonic analysis of non-compact Lie groups. In appendix $\mathrm{C}$ we collect counterparts of some of the results in the trivial case of a flat symmetric space, for the sake of contradistinction. For further orientation we refer to the table of contents. 


\section{Group decomposition of the state space}

Here we introduce the class of systems considered and prepare an orbit decomposition of the configuration manifold. The wave functions (square integrable functions on this manifold) are then subjected to a Plancherel decomposition with respect to their global position, which is parameterized by an element of $G$. Eventually this gives rise to a decomposition of the state space $L^{2}(\mathcal{M})$ into fibers labeled by irreducible unitary representations of the original group action.

\subsection{Generalized spin systems}

We consider generalized spin systems of the following type: the dynamical variables take values in an indecomposable Riemannian symmetric space $Q:=G / K$, with $G$ a noncompact Lie group and $K$ a maximal compact subgroup. The Lie groups will be taken to be linear reductive, meaning that $G$ is a closed subgroup of $\mathrm{GL}(N, \mathbb{R})$ or $\mathrm{GL}(N, \mathbb{C})$ which is stable under conjugate transpose. $K$ then is the isotropy group of the point $q^{\uparrow}=$ $e K \in Q$. We further assume that there is an involution $\iota$ such that $K$ consists of its fixed points, and that $g \iota(g)^{-1}$ has unit determinant. Then $G$ and $K$ form a symmetric pair. Examples are $\mathrm{SO}_{0}(1, N) / \mathrm{SO}(N), \mathrm{SL}(N, \mathbb{R}) / \mathrm{SO}(N), \mathrm{U}(p, q) / \mathrm{U}(p) \times \mathrm{U}(q)$. In Appendix $\mathrm{C}$ we will also consider the degenerate case $\mathbb{R}^{N}=\operatorname{ISO}(N) / \operatorname{SO}(N)$. The configuration manifold $\mathcal{M}$ is the direct product of $\nu:=|\Lambda| \geq 2$ copies of this space. For much of the following $\Lambda$ only has to have the structure of a point set; it is assumed though that $\nu \rightarrow \infty$ captures the physical intuition of a thermodynamic limit. A hypercubical lattice $\Lambda \subset \mathbb{Z}^{d}$ of arbitrary dimension $d$ is a prime example, however neither the dimension nor the structure of the lattice is essential. Ordering the points in some way, we write $m=\left(q_{1}, \ldots, q_{\nu}\right)$ for the points in $\mathcal{M}$. Further we denote by $\gamma_{Q}$ and $d \gamma_{Q}$ the invariant metric and the measure on $Q$. Equipped with the product metric $\gamma(m):=\prod_{i} \gamma_{Q}\left(q_{i}\right)$ and the product measure $d \gamma(m):=\prod_{i} d \gamma_{Q}\left(q_{i}\right)$ the configuration space $\mathcal{M}$ is a simply connected Riemannian manifold, and in fact a reducible Riemannian symmetric space. Further $\mathcal{M}$ carries an action $d: G \times \mathcal{M} \rightarrow \mathcal{M}$ of the group $G$, via $d(g)(m)=\left(g q_{1}, \ldots g q_{\nu}\right)$, where $q \rightarrow g q$ is the left (transitive) action of $G$ on $Q$. Clearly $d(g)$ is an isometry and $d(g)(m)=m$ for all $m$ implies that $g$ is the identity in $G$, that is, the action of $G$ is effective. Since $d(G):=\{d(g), g \in G\}$ is a subgroup of the full isometry group which is closed in the compact-open topology, $(\mathcal{M}, \gamma)$ also is a proper Riemannian $G$-manifold in the sense of [35], Section 5. In fact, the main reason for considering product manifolds of the above type is that they have a well defined orbit decompositon $\mathcal{M}=Q \times \mathcal{N}$, $\mathcal{N} / d(K)=\mathcal{M} / d(G)$, to be described later. With certain refinements this generalizes to all proper Riemannian $G$-manifolds, see [35].

The pure states of the system are described by elements of $L^{2}(\mathcal{M})$, i.e. functions $\psi$ : $\mathcal{M} \rightarrow \mathbb{C}$, square integrable with respect to $d \gamma$. The proper $G$ action $d$ on $\mathcal{M}$ induces

a unitary representation $\ell_{\mathcal{M}}$ of $G$ on $L^{2}(\mathcal{M})$ via $\left(\ell_{\mathcal{M}}(g) \psi\right)(m)=\psi\left(d\left(g^{-1}\right)(m)\right)$. Since 
$L^{2}(\mathcal{M}) \simeq\left[L^{2}(Q)\right]^{\otimes \nu}$ it can be identified with the $\nu$-fold inner tensor product of the left quasi-regular representation $\ell_{1}$ of $G$ on $L^{2}(Q)$; we write $\ell_{\mathcal{M}} \simeq \ell_{1}^{\otimes \nu}$.

As outlined before, we call a bounded selfadjoint $\mathbf{T}$ on $L^{2}(\mathcal{M})$ a transfer operator if it is positive as well as positivity improving. Positivity of $\mathbf{T}$ means $(\psi, \mathbf{T} \psi) \geq 0$, which is equivalent to the spectrum being nonnegative. Typical transfer operators are also positivity improving because they arise as integral operators with a positive kernel; the formalization as a positivity improving map turned out to be useful, see [44 p.201 ff. For convenience we recall the definitions: a function $\mathcal{M} \ni m \mapsto \psi(m) \in \mathbb{C}$ has some property almost everywhere (a.e.) if it holds for all $m \in \mathcal{M} \backslash I$ with $\gamma(I)=0$. A nonzero function is called positive if $\psi \geq 0$ a.e. and strictly positive if $\psi>0$ a.e. Then $\mathbf{T}$ is called positivity preserving if $(\mathbf{T} \psi)(m) \geq 0$ a.e. and positivity improving if $(\mathbf{T} \psi)(m)>0$ a.e. for any positive $\psi$. Equivalently $\mathbf{T}$ is positivity improving iff $(\phi, \mathbf{T} \psi)>0$ for all positive $\phi, \psi \in L^{2}$; see [4] p.202.

Our notion of transfer operators requires an additional condition:

Definition 2.1. A transfer operator $\mathbf{T}$ is a positive integral operator, given by

$$
(\mathbf{T} \psi)(m)=\int d \gamma\left(m^{\prime}\right) T\left(m, m^{\prime}\right) \psi\left(m^{\prime}\right)
$$

where the kernel $T: \mathcal{M} \times \mathcal{M} \rightarrow \mathbb{R}_{+}$is symmetric, continuous and strictly positive, i.e. $T\left(m, m^{\prime}\right)>0$ a.e. and satisfies

$$
\sup _{m} \int d \gamma\left(m^{\prime}\right) T\left(m, m^{\prime}\right)<\infty
$$

The second condition is sufficient (but by no means necessary) to ensure that $\mathbf{T}$ defines a bounded operator from $L^{p}$ to $L^{p}$ for $1 \leq p \leq \infty$; see 30 p.173 ff. The operator norm $\|\mathbf{T}\|_{L^{p} \rightarrow L^{p}}=\sup _{\|\phi\|_{p}=1}\|\mathbf{T} \phi\|_{p}$ is bounded by the integral in (2.2) and coincides with it for $p=1, \infty$. Positivity of the kernel entails that $\mathbf{T}$ is positivity improving. Positivity of the operator (that is, of its spectrum) does not follow from this. However if it is not satisfied we can switch to $\mathbf{T}^{2}$ and the associated integral kernel, where positivity is manifest. Without much loss of generality we assume therefore the kernel to be such that $\mathbf{T}$ is positive. As a bounded symmetric operator on $L^{2}$ the integral operator defined by $T\left(m, m^{\prime}\right)$ has a unique selfadjoint extension which we denote by the same symbol $\mathbf{T}$. The kernel of $\mathbf{T}^{t}$ will be denoted by $T\left(m, m^{\prime} ; t\right)$ for $t \in \mathbb{N}$. In this situation $\mathbf{T}$ and all its powers are transfer operators in the sense of the previous definition.

An invariant dynamics is specified by a $G$-invariant transfer operator, i.e. one which commutes with $\ell_{\mathcal{M}}$ on $L^{2}$

$$
\ell_{\mathcal{M}}(g) \circ \mathbf{T}=\mathbf{T} \circ \ell_{\mathcal{M}}(g), \quad \forall g \in G
$$

It is easy to see that $\mathbf{T}$ then cannot have normalizable ground states (see Proposition 3.1 below). In this situation one will naturally search for generalized eigenstates of $\mathbf{T}$, 
which in our case will be simply solutions of $\mathbf{T} \Omega=\|\mathbf{T}\| \Omega$ with $\Omega$ an almost everywhere defined function (not just a distribution) on $\mathcal{M}$. The set of generalized ground states forms a linear space which we call the ground state sector $\mathcal{G}(\mathbf{T})$ of $\mathbf{T}$.

\subsection{The spectral problem}

Since $\mathbf{T}$ commutes with the $G$ action $\ell_{\mathcal{M}}$, one expects that the transfer operator and a set of operators $\mathcal{Z}$ whose diagonal action characterizes an irreducible representation can be diagonalized simultaneously. In a sense this is correct and the generalized eigenfunctions with spectral value $\|\mathbf{T}\|$ in fact belong to a special irreducible representation of $G$, see Theorem 1.1. The purpose of this interlude is to explain why the general results available in the literature on such (simultaneous) spectral decompositions are insufficient to produce the generalized ground states sought for.

Let $\mathbf{A}$ be a bounded selfadjoint operator on the separable Hilbert space $L^{2}(\mathcal{M})$. Let $\Phi \subset L^{2}(\mathcal{M}) \subset \Phi^{\prime}$ be a Gel'fand triple [17, 6] (rigged Hilbert space) for A. An element $\Omega \in \Phi^{\prime}$ is called an eigendistribution or generalized eigenstate of $\mathbf{A}$ with spectral value $\lambda \in \operatorname{Spec}(\mathbf{A})$ if $(\phi,(\mathbf{A}-\lambda) \Omega)=0$, for all $\phi \in \Phi$. The set of generalized eigenstates for some $\lambda \in \operatorname{Spec}(\mathbf{A})$ forms a linear subspace of $\Phi^{\prime}$ which is called the generalized eigenspace $\mathcal{E}_{\lambda}(\mathbf{A})$ for the spectral value $\lambda \in \operatorname{Spec}(\mathbf{A})$. If $L^{2}(\mathcal{M})$ carries the unitary representation $\ell_{\mathcal{M}}$ of a connected (noncompact) Lie group group $G$ there is a natural action of $G$ on the distributions $\Omega \in \Phi^{\prime}$, viz $\left(\ell_{\mathcal{M}}(g) \circ \Omega, \phi\right):=\left(\Omega, \ell_{\mathcal{M}}(g)^{-1} \circ \phi\right)$ for all $\phi \in \Phi$ and $g \in G$. Naturally $\Omega$ is called invariant if $\ell_{\mathcal{M}}(g) \circ \Omega=\Omega$ for all $g \in G$. If $\mathbf{A}$ commutes with $\ell_{\mathcal{M}}, \ell_{\mathcal{M}}(g) \circ \mathbf{A}=\mathbf{A} \circ \ell_{\mathcal{M}}(g)$ for all $g \in G$, one expects that the generalized eigenspaces can be decomposed into components irreducible with respect to $\ell_{\mathcal{M}}$. Under mild extra conditions this is indeed the case. The nuclear spectral theorem (Gel'fandMaurin theorem, [17, 34]) guarantees the existence of direct integral decompositions of the form

$$
L^{2}(\mathcal{M})=\int_{\operatorname{Spec}(\mathbf{A}) \times \widehat{G}} d \mu(\lambda, \sigma) \mathcal{E}_{\lambda, \sigma}(\mathbf{A}) .
$$

Here $\widehat{G}$ is the dual of $G$ and $\mu(\lambda, \sigma)$ is a measure on $\operatorname{Spec}(\mathbf{A}) \times \widehat{G}$ defining the decomposition. To simplify the notation we identified elements $\pi_{\sigma}$ of $\widehat{G}$ with a set of parameters $\sigma$ uniquely specifying an equivalence class of unitary irreducible representations. The precise version of the nuclear spectral theorem can be found in [17, 34, 6]. The fiber spaces $\mathcal{E}_{\lambda, \sigma}(\mathbf{A})$ contain the generalized eigenfunctions of $\mathbf{A}$ in $\Phi^{\prime}$ transforming irreducibly under $G$. The nuclear spaces $\Phi$ are much smaller than $L^{1}$, the dual spaces $\Phi^{\prime}$ therefore much larger than $L^{\infty}$, and the generalized eigenfunctions supplied by the Gel'fand type constructions may be genuine distributions. The fact that $\mathbf{A}$ commutes with the elliptic Nelson operator of $G$ (built from the Casimirs of $G$ and $K$ ) entails 34 that the (averaged) eigendistributions $\ell_{\mathcal{M}}(g) \circ \Omega$ are smooth functions in $g$, but little can be said about their distributional type. A result by Berezanskii (described and proven in [34]) specifies sufficient conditions under which the dual space $\Phi^{\prime}$ of a triple $\Phi \subset L^{2} \subset \Phi^{\prime}$ 
consists of almost everywhere defined functions (not distributions). We shall make use of this result for the 'relative motion' alluded to in the introduction. Irrespective of the distributional type of the generalized eigenfunctions the decomposition (2.4) has however two important drawbacks:

- generalized eigenfunctions are assured to exist only for $\mu$-almost all spectral values $\lambda \in \operatorname{Spec}(\mathbf{A})$, and the measure $\mu$ is usually not known explicitly.

- whenever generalized eigenfunctions exist for a given $\lambda \in \operatorname{Spec}(\mathbf{A})$, let $\widehat{G}_{\lambda} \subset \widehat{G}$ denote a set that carries the restricted measure in (2.4). Then the spaces $\mathcal{E}_{\lambda, \sigma}(\mathbf{A})$ are assured to be irreducible only for $\mu$-almost all $\sigma \in \widehat{G}_{\lambda}$.

The first of these 'almost all' caveats presents a major obstruction if one wants to apply the general framework to a specific spectral value, like $\|\mathbf{T}\|$, the ground state value, which is our main concern here.

The following example illustrates the problem. Let $\mathbf{T}$ be the integral operator on $L^{2}\left(\mathbb{R}_{+}\right)$ defined by the kernel $\mathcal{T}(x, y)=e^{-|x-y|}$. It can be seen to be a transfer operator in the above sense with spectrum $\operatorname{Spec}(\mathbf{T})=[0,2]$. For all spectral values different from $\lambda=2$ there exist generalized $L^{\infty}$ eigenstates, yet the operator does not have a generalized ground state. The point to observe is that for all $\psi \in L^{2}\left(\mathbb{R}_{+}\right)$the image function $(\mathbf{T} \psi)(x)$ is twice differentiable with

$$
(\mathbf{T} \psi)^{\prime \prime}=(\mathbf{T} \psi)-2 \psi
$$

All solutions of $\mathbf{T} \psi=\lambda \psi$ therefore must be linear combinations of $e^{ \pm i \omega x}$ with $\omega=$ $\sqrt{2 / \lambda(\omega)-1}$, i.e. $\lambda(\omega)=2 /\left(1+\omega^{2}\right)$. One finds

$$
\psi_{\omega}(x)=\frac{1}{\sqrt{1+\omega^{2}}}[\sin \omega x+\omega \cos \omega x]=\cos (\omega x-b), \quad \omega \geq 0, \quad b=\operatorname{arccot} \omega
$$

where the normalization has been chosen such that $\left\|\psi_{\omega}\right\|_{\infty}=1$. The explicit construction shows that $\psi_{\omega} \in L^{\infty}$, although the space of test functions is slightly smaller than $L^{1}$ in that twice differentiable $L^{1}$ functions $\phi$ have to satisfy $\phi(0)=\partial_{x} \phi(0)$ (which can be seen by averaging Eq. (2.5) with a test function). The fact that the generalized eigenfunctions (2.6) also satisfy $\psi_{\omega}(0)=\partial_{x} \psi_{\omega}(0)$ ensures their completeness; it is easy to verify that

$$
\begin{aligned}
& \int_{0}^{\infty} d \omega \psi_{\omega}(x) \psi_{\omega}(y)=\frac{\pi}{2} \delta(x-y), \quad x, y \geq 0 \\
& \int_{0}^{\infty} d x \psi_{\omega_{1}}(x) \psi_{\omega_{2}}(x)=0, \quad \omega_{1} \neq \omega_{2} .
\end{aligned}
$$

Using Eq. (2.7) we find the following spectral resolution of the integral kernel $\mathcal{T}$ :

$$
\mathcal{T}(x, y ; n)=\frac{2}{\pi} \int_{0}^{\infty} d \omega\left(\frac{2}{1+\omega^{2}}\right)^{n} \psi_{\omega}(x) \psi_{\omega}(y)
$$


For example

$$
\mathcal{T}(x, y ; 2)=e^{-|x-y|}(1+|x-y|)-\frac{1}{2} e^{-(x+y)} .
$$

Despite these nice properties no generalized ground state exists. This is because candidates for it must be contained in the set (2.6); however the relevant limit $(\lambda \rightarrow 2$ i.e. $\omega \rightarrow 0)$ vanishes pointwise, while the closest maximum of $\psi_{\omega}$, lying at $b(\omega) / \omega \sim$ $\pi /(2 \omega)$, moves out to $\infty$.

The upshot is that the 'almost all' caveat in the general theorems is crucial for their validity and renders them at the same time useless for the construction e.g. of the ground state sector. Even transfer operators with a complete system of regular (here: $\left.L^{\infty}\right)$ generalized eigenfunctions may fail to have a ground state. This explains why in the constructive Theorem 5.1 certain subsidiary conditions must be present; we do expect however that the ones given can still be weakened.

In Section 3 we will analyze the spectral problem for invariant selfadjoint operators $\mathbf{A}$ as defined in Definition 3.1. Under mild subsidiary conditions $((\mathrm{C})$ in Section 3.3 and $(\mathrm{C} 1)$, (C2) in Section 3.4) a complete set of eigenfunctions $\Omega_{\lambda \sigma}$ in $\mathcal{E}_{\lambda \sigma}(\mathbf{A})$ can be found. Some of the properties of the $\Omega_{\lambda \sigma}$ have been anticipated in the introduction. In this context it is worth emphazising two points. First, in contrast to the familar situation with normalizable eigenfunctions the existence of a $\Omega \in \Phi^{\prime}$ such that $\mathbf{A} \Omega=\lambda \Omega$ does in itself not imply $\lambda \in \operatorname{Spec}(\mathbf{A})$. A simple counterexample is the hyperbolic spin chain discussed in detail in [38]: there the constant functions are eigenfunctions of the transfer operator $\mathbf{T}$, but the corresponding eigenvalue lies above the spectrum of $\mathbf{T}$. When solving the spectral problem $\mathbf{A} \Omega=\lambda \Omega$ with a nonnormalizable $\Omega \in \Phi^{\prime}$, the information that $\lambda$ is a point in the spectrum therefore has to be supplied independently. A second point worth repeating is that all known construction principles for generalized eigenfunctions (including the one presented in Section 3) are guaranteed to work only for almost all points in the spectrum. For a prescribed $\lambda \in \operatorname{Spec}(\mathbf{A})$ additional considerations are necessary to show that sufficiently many eigenfunctions exist. This applies in particular to the $\sigma$-equivariant eigenspaces $\mathcal{E}_{\lambda \sigma}(\mathbf{A})$ of an invariant selfadjoint operator $\mathbf{A}$ and to the ground state fibers $\mathcal{G}_{\sigma}(\mathbf{T}):=\mathcal{E}_{\|\mathbf{T}\|, \sigma}(\mathbf{T})$ of a transfer operator.

Whenever the fiber spaces $\mathcal{E}_{\lambda, \sigma}(\mathbf{A})$ in (2.4) are nonempty for a fixed $\lambda \in \operatorname{Spec}(\mathbf{A})$ one can match the decomposition in (2.4) with the purely group theoretical one. Since $\ell_{\mathcal{M}}$ is a unitary representation on general grounds it can be decomposed into irreducible components 9]. That is, there exists a measure $\mu_{\ell_{\mathcal{M}}}$ on $\widehat{G}$ such that

$$
L^{2}(\mathcal{M})=\int_{\widehat{G}}^{\oplus} d \mu_{\ell_{\mathcal{M}}}(\sigma) \mathcal{L}_{\sigma}^{2}(\mathcal{M})
$$

where the fibers $\mathcal{L}_{\sigma}^{2}(\mathcal{M})$ are irreducible for $\mu_{\ell_{\mathcal{M}}}$-almost all $\sigma \in \widehat{G}$ in the support of the measure. On the other hand from (2.4) one can define generalized eigenspaces $\mathcal{E}_{\lambda}(\mathbf{A})$ by 


$$
\mathcal{E}_{\lambda}(\mathbf{A})=\int_{\widehat{G}_{\lambda}} d \mu(\lambda, \sigma) \mathcal{E}_{\lambda, \sigma}(\mathbf{A}),
$$

where $\widehat{G}_{\lambda}$ denotes the part of $\widehat{G}$ for which there is a non-empty eigenspace $\mathcal{E}_{\lambda, \sigma}(\mathbf{A})$.

Thus $\int_{\operatorname{Spec}(\mathbf{A})} d \mu(\lambda, \sigma)=d \mu_{\ell_{\mathcal{M}}}(\sigma)$. There are no obvious strategies to determine the representation content $\widehat{G}_{\lambda} \subset \widehat{G}$ of a given spectral value $\lambda$ (for which generalized eigenfunctions exist). For a transfer operator $\mathbf{T}$ we identify its ground state sector $\mathcal{G}(\mathbf{T})$ with $\mathcal{E}_{\|\mathbf{T}\|}(\mathbf{T})$, and similarly for the equivariant fibers $\mathcal{G}_{\sigma}(\mathbf{T})=\mathcal{E}_{\|T\|, \sigma}(\mathbf{T})$. We shall thus apply the decomposition (2.11) also for the ground state sector and write $\widehat{G}_{\|T\|}$ for its representation content. One of the main goals later on will be to show that under moderate extra assumptions $\widehat{G}_{\|T\|}$ consists of a single point (a single representation) only, which is always the same for all transfer operators considered.

\subsection{Orbit decomposition}

A simple but crucial fact about the configuration manifolds $\mathcal{M}=Q \times \ldots \times Q$ is that they have a well defined orbit decomposition which eventually carries over to the states and the operators acting on them. The idea of the decomposition is to single out one of the variables in $m=\left(q_{1}, \ldots, q_{\nu}\right)$, say $q_{1}$, to parameterize the location on the orbits and to define coordinate functions $n_{i}$ transversal to it to describe the relative location of the points such that they change only by elements of $K$ as one moves along an orbit. To this end we fix some $q^{\uparrow} \in Q=e K$ with isotropy group $K$, i.e. $k q^{\uparrow}=q^{\uparrow}$ for all $k \in K$. Based on it we wish to define a section $g_{s}: Q \rightarrow G$ such that $q=g_{s}(q) q^{\uparrow}$ for all $q \in Q$. Clearly this condition defines $g_{s}$ only up to right multiplication by some $k_{s}=k_{s}(g, q) \in K$,

$$
g_{s}(g q)=g g_{s}(q) k_{s}(g, q)
$$

For $g_{s}$ to be well-defined the element $k$ has to be uniquely determined for given $g$ and $q$. It is easy to see that this is the case whenever $G$ admits an Iwasawa decomposition, which is the case for all connected simple noncompact Lie groups, in particular the ones considered. Consistency requires the cocycle condition $k_{s}\left(g_{1} g_{2}, q\right)=k_{s}\left(g_{2}, q\right) k_{s}\left(g_{1}, g_{2} q\right)$, in particular $k_{s}(e, q)=e$ for all $q \in Q$ and $e \in G$ the identity. If we normalize $g_{s}$ such that $g_{s}\left(q^{\uparrow}\right)=e$ it follows that $k_{s}\left(g_{s}(q), q^{\uparrow}\right)=e=k_{s}\left(g_{s}(q)^{-1}, q\right)$ and $k_{s}(g, q)=k_{s}\left(g g_{s}(q), q^{\uparrow}\right)$. For elements $k \in K$ of the subgroup one has $k_{s}\left(k, q^{\uparrow}\right)=k^{-1}$. Generally the Iwasawa decomposition entails that the cocycle $k_{s}(\cdot, q): G \rightarrow K$ is surjective for all $q \in Q$.

Our main example for the symmetric space $Q$ will be $\mathbb{H}_{N}=\mathrm{SO}_{0}(1, N) / \mathrm{SO}(N)$, the $N$-dimensional hyperboloid with the Riemannian metric $\gamma_{\mathbb{H}_{N}}$ induced by the indefinite metric $q \cdot q=\left(q^{0}\right)^{2}-\left(q^{1}\right)^{2}-\ldots-\left(q^{N}\right)^{2}$ in the imbedding linear space. Explicitly $\mathbb{H}_{N}=\left\{q \in \mathbb{R}^{1, N} \mid q \cdot q=\left(q^{0}\right)^{2}-\left(q^{1}\right)^{2}-\ldots-\left(q^{N}\right)^{2}=1, q^{0}>0\right\}$. The invariant measure on $\mathbb{H}_{N}$ is $d \gamma_{\mathbb{H}_{N}}(q)=d^{N+1} q \delta\left(q^{2}-1\right) \theta\left(q^{0}\right)$ and will be denoted by $d q$ for short. The 
$\nu$-fold product $\mathcal{M}=\mathbb{H}_{N}^{\nu}$ equipped with the product metric then is a simply connected Riemannian manifold (and, in fact, a reducible symmetric space). We denote the product measure $\prod_{i=1}^{\nu} d q_{i}$ on $\mathcal{M}$ by $d \gamma_{\mathcal{M}}$. For $G=\mathrm{SO}_{0}(1, N)$ and $K=\mathrm{SO}(N)$ the section $g_{s}(q)$ is just the familar expression for the pure boost mapping $q^{\uparrow}=(1,0, \ldots, 0)$ to $q=\left(q_{0}, q_{1}, \ldots, q_{N-1}\right)=:\left(q_{0}, \vec{q}\right)$. Explicitly

$$
g_{s}(q)=\left(\begin{array}{c|c}
q_{0} & \vec{q}^{T} \\
\hline \vec{q} & \mathbb{I}+\frac{1}{q_{0}+1} \vec{q} \vec{q}^{T}
\end{array}\right) .
$$

It satisfies $g_{s}(q)=e$ iff $q=q^{\uparrow}$. The cocycle $k_{s}(g, q)=g_{s}(q)^{-1} g^{-1} g_{s}(g q)$ based on this section is known as 'Wigner rotation' [59] and satisfies

$$
k_{s}(k, q)=k^{-1} \quad \text { for all } q \in Q, k \in K,
$$

that is, not only for $q=q^{\uparrow}$.

Using the section $g_{s}$ we now define the following diffeomorphism on $\mathcal{M}=Q^{\nu}$ :

$$
\begin{aligned}
& \vartheta\left(q_{1}, \ldots, q_{\nu}\right)=\left(q_{1}, g_{s}\left(q_{1}\right)^{-1} q_{2}, \ldots, g_{s}\left(q_{1}\right)^{-1} q_{\nu}\right)=:\left(q_{1}, n_{2}, \ldots, n_{\nu}\right), \\
& \vartheta^{-1}\left(q_{1}, n_{2}, \ldots, n_{\nu}\right)=\left(q_{1}, g_{s}\left(q_{1}\right) n_{2}, \ldots, g_{s}\left(q_{1}\right) n_{\nu}\right) .
\end{aligned}
$$

This diffeomorhism is measure preserving due to the invariance of the measures $d q_{i}$ : let $f \in L^{1}\left(d \gamma_{\mathcal{M}}\right)$ and $d n:=d \gamma_{\mathcal{N}}(n):=\prod_{i \neq 1} d \gamma_{Q}\left(n_{i}\right)$. Then

$$
\begin{aligned}
& \int(f \circ \vartheta)\left(q_{1}, n\right) d \gamma_{\mathcal{M}}(m)=\int d q_{1} \int \prod_{i \neq 1} d q_{i} f\left(q_{1}, g_{s}\left(q_{1}\right)^{-1} q_{2}, \ldots, g_{s}\left(q_{1}\right)^{-1} q_{\nu}\right) \\
& =\int d q_{1} \int \prod_{i \neq 1} d q_{i} f\left(q_{1}, \ldots, q_{\nu}\right)=\int d \gamma_{\mathcal{M}}(m) f(m) .
\end{aligned}
$$

So the measure $d \gamma_{\mathcal{M}}$ can also be factorized as

$$
d \gamma_{\mathcal{M}}(m)=d q_{1} d n
$$

The product $Q \times \mathcal{N}$ equipped with the product metric $\gamma_{Q} \times \gamma_{\mathcal{N}}$ and the measure $d q d n$ is a Riemannian manifold $\mathcal{M}_{s}$ which by construction is isometric to $\mathcal{M}$, and with the isometry given by the above $\vartheta$ :

$$
\vartheta:(\mathcal{M}, \gamma) \longrightarrow\left(\mathcal{M}_{s}, \gamma_{s}\right):=\left(Q \times \mathcal{N}, \gamma_{Q} \times \gamma_{\mathcal{N}}\right)
$$

The manifold $\mathcal{M}_{s}$ also has the structure of a $G$ space which it inherits from $\mathcal{M}$. Recall that the transversal coordinate functions $n_{i}=n_{i}\left(q_{1}, q_{i}\right) \in Q$ are defined by 
$n_{i}:=g_{s}\left(q_{1}\right)^{-1} q_{i}$, for $i=2, \ldots, \nu$. On them $\ell_{\mathcal{M}}$ acts by $\ell_{\mathcal{M}}(g)\left(n_{i}\right)=g_{s}\left(g^{-1} q_{1}\right)^{-1} g^{-1} q_{i}=$ $k_{s}\left(g^{-1}, q_{1}\right)^{-1} n_{i}$. The original diagonal action $d(g) m=\left(g q_{1}, \ldots, g q_{\nu}\right)$ becomes a twisted action $d_{s}$ (depending on a choice of section) in the coordinates $(q, n)$, i.e. $\vartheta \circ d=d_{s} \circ \vartheta$, with

$$
d_{s}\left(g^{-1}\right)(q, n)=\left(g^{-1} q, k_{s}\left(g^{-1}, q\right)^{-1} n\right) .
$$

As $d_{s}\left(g_{s}(q)\right)\left(q_{0}, n\right)=(q, n)$ and $d_{s}\left(g_{s}(q)^{-1}\right)(q, n)=\left(q_{0}, n\right)$ it acts transitively on the first variable. However as one moves along $Q$ the action $d_{s}$ co-rotates the $n$ variables in a $q$-dependent way. For a generic symmetric space this happens even on the subgroup $K$; for $\mathbb{H}_{N}$ and the Wigner rotation one has $d_{s}\left(k^{-1}\right)(q, n)=\left(k^{-1} q, k^{-1} n\right)$, though. In the terminology of [60], Section $4, \mathcal{M}_{s}:=Q \times_{k_{s}} \mathcal{N}$ is the skew product $G$-space induced from the $K$-space $\mathcal{N}$. Indeed, $\mathcal{N}$ equipped with the diagonal action of $K: d_{\mathcal{N}}(k) n=$ $\left(k^{-1} n_{2}, \ldots, k^{-1} n_{\nu}\right)$ is a $K$-space, and by construction $(\mathcal{M}, d)$ and $\left(\mathcal{M}_{s}, d_{s}\right)$ are isometric as $G$ spaces. Since the cocycle is surjective for fixed $q$ we gained a less redundant description of the space of orbits:

$$
\text { space of orbits: } \mathcal{M} / d(G)=\mathcal{M}_{s} / d_{s}(G)=\mathcal{N} / d_{\mathcal{N}}(K)
$$

where, importantly, $K$ is compact. On the other hand, the twisted action (2.19) is cumbersome when one tries to decompose the unitary representation based on $d_{s}$ into irreducible components. But the left twisted action $d_{s}$ can be traded for an untwisted right action $r$ by the following construction (based on a remark in [60], p.75). Consider

$$
\mathcal{M}_{r}:=(G \times \mathcal{N}) / d(K)
$$

that is, the space of equivalence classes $(g, n) \sim\left(k^{-1} g, k^{-1} n\right), k \in K$ in $G \times \mathcal{N}$. In order not to clutter the notation we also write $(g, n)$ for the equivalence class generated by a point in $G \times \mathcal{N}$. Maps and functions on $G \times \mathcal{N}$ that are constant on $d(K)$ orbits then lift unambiguously to maps and functions on $\mathcal{M}_{r}$. On $G \times \mathcal{N}$ and $\mathcal{M}_{r}$ we define a right $G$-action $r$ by

$$
r\left(g^{\prime}\right)(g, n)=\left(g g^{\prime}, n\right),
$$

which is just the standard right action of $G$ on itself leaving the $n$ variables untouched. The action (2.22) is constant on the equivalence classes because the right $r(G)$ action and the left $d(K)$ action on $G \times \mathcal{N}$ commute, $r(g) d(k)=d(k) r(g)$. In fact $\mathcal{M}_{r}$ equipped with the right $G$ action is isomorphic to the original manifold $\mathcal{M}=Q^{\nu}$ with the diagonal left action $\ell_{\mathcal{M}}(G)$ (and thereby also to the skew product $G$ space $\mathcal{M}_{s}=Q \times_{k_{s}} \mathcal{N}$ ). The isomorphism is given by first considering the following map $\tilde{\phi}: \mathcal{M} \rightarrow G \times \mathcal{N}$ :

$$
\begin{aligned}
& \tilde{\phi}: \mathcal{M} \longrightarrow G \times \mathcal{N}, \\
& \tilde{\phi}\left(q_{1}, \ldots, q_{\nu}\right)=\left(g_{s}\left(q_{1}\right)^{-1}, g_{s}\left(q_{1}\right)^{-1} q_{2} \ldots, g_{s}\left(q_{1}\right)^{-1} q_{\nu}\right) .
\end{aligned}
$$

This map is injective, but not surjective. Because $g_{s}$ is a global section of $G / K$ its range intersects each $d(K)$ orbit exactly once, so that it determines uniquely a diffeomorphism 


$$
\phi: \mathcal{M} \longrightarrow \mathcal{M}_{r}
$$

We define an inverse of $\tilde{\phi}^{-1}: G \times \mathcal{N} \longrightarrow \mathcal{M}$ (initially only defined on the range of $\tilde{\phi}$ ) by

$$
\tilde{\phi}^{-1}(g, n)=d\left(g^{-1}\right)\left(q^{\uparrow}, n\right) .
$$

We can immediately interpret this as a map from all of $G \times \mathcal{N}$ to $\mathcal{M}$ which is constant on the equivalence classes under $d(K)$

$$
\tilde{\phi}^{-1}(k g, k n)=d(k g)\left(q^{\uparrow}, k n\right)=\left(g^{-1} q^{\uparrow}, g^{-1} n\right)=\tilde{\phi}^{-1}(g, n),
$$

and hence lifts to a map $\phi^{-1}: \mathcal{M}_{r} \longrightarrow \mathcal{M}$. By direct computation one verifies

$$
\tilde{\phi} \circ \tilde{\phi}^{-1}=\mathrm{id}
$$

whereas $\tilde{\phi}^{-1} \circ \tilde{\phi}$ only maps orbits of $d(K)$ into themselves. But this is enough to see that $\phi$ and $\phi^{-1}$ are really inverse to each other.

According to (2.22) the map $\phi$ intertwines the left action $d(G)$ with the right action $r(G)$ :

$$
\phi \circ d=r \circ \phi \text {. }
$$

In addition the map $\phi$ is measure preserving; this can be seen similarly as the measure preserving property of $\vartheta$ : consider a $L^{1}$ function $f$ on $\mathcal{M}$ and let $f_{r}=f \circ \phi^{-1}$. Then, using the $G$-invariance of the measure $d n$ and the fact that the invariant measure $d q$ is the push-forward of Haar measure $d g$ under the canonical projection $G \longrightarrow G / K$, one sees that

$$
\int \prod_{i=1}^{\nu} d \gamma_{Q}\left(q_{i}\right) f\left(q_{1}, \ldots, q_{\nu}\right)=\int d g d n f_{r}(g, n) \text {. }
$$

For completeness we also note explicitly the isometry $\chi=\phi \circ \vartheta^{-1}$ between the skew product $Q$ space $\mathcal{M}_{s}=Q \times_{k_{s}} \mathcal{N}$ and the $G$-manifold $\mathcal{M}_{r}$ with the diagonal right action:

$$
\begin{aligned}
\chi: Q \times_{k_{s}} \mathcal{N} \rightarrow \mathcal{M}_{r}, \quad \chi(q, n) & =\left(g_{s}(q)^{-1}, n\right), \\
\chi^{-1}: \mathcal{M}_{r} \rightarrow Q \times_{k_{s}} \mathcal{N}, \quad \chi^{-1}(g, n) & =\left(g^{-1} q^{\uparrow}, k_{s}\left(g^{-1}, q^{\uparrow}\right)^{-1} n\right) .
\end{aligned}
$$

In summary we have two equivalent descriptions of the original $G$-manifold $(\mathcal{M}, d)$, namely $\left(\mathcal{M}_{s}, d_{s}\right)$ and $\left(\mathcal{M}_{r}, r\right)$. The second one is more convenient for the reduction problem because the space of orbits $\mathcal{M} / d(G)=\mathcal{M}_{r} / r(G)$ is now described by equivalence classes with respect to the usual right action of $G$ on itself. 
This structure of course carries over to the function spaces and the unitary representations on them induced by the G-actions. We have

$$
\begin{aligned}
L^{2}(\mathcal{M}) \ni \psi & \mapsto\left(\ell_{\mathcal{M}}(g) \psi\right)(m)=\psi\left(d\left(g^{-1}\right)(m)\right), \\
L^{2}\left(\mathcal{M}_{s}\right) \ni \psi_{s} & \mapsto\left(\ell_{s}(g) \psi_{s}\right)(q, n)=\psi_{s}\left(d_{s}\left(g^{-1}\right)(q, n),\right. \\
L^{2}\left(\mathcal{M}_{r}\right) \ni \psi_{r} & \mapsto\left(\rho\left(g_{0}\right) \psi_{r}\right)(g, n)=\psi_{r}\left(r\left(g_{0}\right)(g, n)\right), \quad \ell_{r}(K) \psi_{r}=\psi_{r},
\end{aligned}
$$

where $\ell_{r}(k) \psi_{r}(g, n)=\psi_{r}\left(k^{-1} g, k^{-1} n\right)$. As Hilbert spaces of course all three $L^{2}$ spaces are isometric to $L^{2}(Q \times \mathcal{N})$ and the three representations $\ell_{\mathcal{M}}, \ell_{s}$, and $\rho$ are likewise unitarily equivalent. Explicitly

$$
\begin{aligned}
& \Phi: L^{2}\left(\mathcal{M}_{r}\right) \longrightarrow L^{2}(\mathcal{M}), \quad\left(\Phi \psi_{r}\right)(m):=\psi_{r}(\phi(m)), \\
& \rho=\Phi^{-1} \circ \ell_{\mathcal{M}} \circ \Phi
\end{aligned}
$$

and similarly for $L^{2}\left(\mathcal{M}_{s}\right)$. By $(2.31)$ invariant under $\ell_{r}(K)$ of $L^{2}(G \times \mathcal{N})$. By (2.29) the map $\Phi$ is indeed an isometry. As $\left(\Phi^{-1} \circ \ell_{\mathcal{M}}\left(g_{0}\right) \circ \Phi \psi_{r}\right)(g, n)=\psi_{r}\left(\phi \circ d\left(g_{0}^{-1}\right) \circ \phi^{-1}(g, n)\right)$ the unitary equivalence of the representations follows from (2.28).

We summarize our results in

Proposition 2.1. There is a diffeomorphism $\phi$ from the configuration manifold $\mathcal{M}=Q^{\nu}$ to $\mathcal{M}_{r}=\left(G \times Q^{\nu-1}\right) / d(K)$ such that the diagonal left action $d(G)$ on $\mathcal{M}$ gets mapped into the right action $r(G)$ on the first factor of $\mathcal{M}_{r}$. $\phi$ is measure preserving for the natural measures on $\mathcal{M}$ and $\mathcal{M}_{r}$ and therefore induces a natural isomorphism of the spaces $L^{2}(\mathcal{M})$ and $L^{2}\left(\mathcal{M}_{r}\right)$.

\subsection{The reduction of $\rho(G)$ on $L^{2}\left(\mathcal{M}_{r}\right)$}

With these preparations at hand we can now address the reduction problem of $\ell_{\mathcal{M}}(G)$ in the variant where it acts as $\rho(G)$ on $L^{2}\left(\mathcal{M}_{r}\right)$. As noted above, the latter is the $\ell_{r}(K)$ invariant subspace of $L^{2}(G \times \mathcal{N})$, which carries the commuting unitary representations $\rho(G)$ and $\ell_{r}(K)$. Moreover $\rho(G)$ for fixed $n \in \mathcal{N}$ is just the right regular representation of $G$ mapping $\psi_{r}(g, n)$ into $\rho\left(g_{0}\right) \psi_{r}(g, n)=\psi_{r}\left(g g_{0}, n\right)$. Its decomposition into unitary irreducible representations $\pi_{\sigma}, \sigma \in \widehat{G}_{r}$, is thus given by the Plancherel decomposition (A.1). The precise form used and the notations are summarized in appendix A. In particular $d \nu$ is the Plancherel measure on $\widehat{G}_{r}$, the restricted dual of $G$, and $g \mapsto \pi_{\sigma}(g)$ denotes the irreducible representation associated with some $\sigma \in \widehat{G}_{r}$. It acts on a separable Hilbert space $\mathcal{L}_{\sigma}$ with inner product $(\cdot, \cdot)_{\sigma}$. 
Applying the expansion (A.2) to the $G$-part of a function in $\psi: G \times \mathcal{N} \rightarrow \mathbb{C}$ gives

$$
\begin{aligned}
\psi(g, n) & =\int_{\widehat{G}_{r}} d \nu(\sigma) \operatorname{Tr}\left[\pi_{\sigma}(g) \widehat{\psi}(\sigma, n)\right] \\
\widehat{\psi}(\sigma, n) & =\int_{G} d g \pi_{\sigma}\left(g^{-1}\right) \psi(g, n)
\end{aligned}
$$

For functions $\psi$ that are $\ell_{r}(K)$ invariant this will lead to the desired decomposition of $L^{2}\left(\mathcal{M}_{r}\right)$ into irreducible components. We consider here first the decomposition of the larger space $L^{2}(G \times \mathcal{N})$. Provided suitable conditions are imposed on the function $\psi$ (which we describe shortly) the transforms $\widehat{\psi}(\sigma, n)$ for fixed $n$ are trace class or HilbertSchmidt operators on $\mathcal{L}_{\sigma}$. Further they satisfy

$$
\left[\rho\left(g_{0}\right) \ell\left(g_{1}\right) \psi\right]^{\hat{}}(\sigma, n)=\pi_{\sigma}\left(g_{0}\right) \widehat{\psi}(\sigma, n) \pi_{\sigma}\left(g_{1}^{-1}\right)=\left(\pi_{\sigma} \times \pi_{\check{\sigma}}\right)\left(g_{0}, g_{1}\right) \widehat{\psi}(\sigma, n)
$$

using (A.6) and the notation $\check{\pi}_{\sigma}=\pi_{\check{\sigma}}$ in the last equation. This states that the map $\psi \rightarrow \widehat{\psi}$ intertwines the outer tensor product $\rho \times \ell$ of the left and the right regular representation of $G$ with $\pi_{\sigma} \times \pi_{\check{\sigma}}$.

Throughout we shall adopt the following conventions for compact operators $A, B$ on some separable Hilbert space $\mathcal{H}$ with orthonormal basis $e_{i}, i \in \mathbb{N}$, and its dual space $\check{\mathcal{H}}$ with dual basis $\check{e}_{i}, i \in \mathbb{N}$ :

$$
A=\sum_{i j} e_{i} A_{i j} \check{e}_{j}, \quad\left[A^{\dagger}\right]_{i j}=A_{j i}^{*}
$$

Compact operators that are even trace class arise for example as Fourier transforms of functions $\psi \in \mathcal{D}$, where $\mathcal{D}$ is the space of functions $\psi(g, n)$ that are smooth with compact support in $g \in G$ and square integrable in $n \in \mathcal{N}$ : for such $\psi$ the Fourier transform $\widehat{\psi}(\sigma, n)$ is a trace class operator on a separable Hilbert space $\mathcal{L}_{\sigma}$, for all $\psi \in \mathcal{D}$, and almost all $\sigma \in \widehat{G}_{r}, n \in \mathcal{N}$. Moreover the Fourier expansion (2.33) then is valid pointwise in $g$. If $\psi$ is in $L^{2}(G) \cap L^{1}(G)$ as a function of $g$ and square integrable in $n$ the Fourier coefficients $\widehat{\psi}(\sigma)$ are still Hilbert-Schmidt operators for almost all $\sigma \in \widehat{G}_{r}, n \in \mathcal{N}$. We identify the trace class operators with a subspace of $\mathcal{L}_{\sigma} \otimes \check{\mathcal{L}}_{\check{\sigma}}$, which in turn can be identified with the space of Hilbert-Schmidt operators on $\mathcal{L}_{\sigma}$; see Appendix A3. This means the coefficients are functions $\widehat{\psi}(\sigma, \cdot): \mathcal{N} \rightarrow \mathcal{L}_{\sigma} \otimes \check{\mathcal{L}}_{\check{\sigma}}$. The Parseval identity

$$
\int_{G} d g \phi(g, n)^{*} \psi\left(g, n^{\prime}\right)=\int_{\widehat{G}_{r}} d \nu(\sigma) \operatorname{Tr}\left[\widehat{\phi}(\sigma, n)^{\dagger} \psi\left(\sigma, n^{\prime}\right)\right],
$$

is valid for all functions in $L^{2}\left(\mathcal{M}_{r}\right)$ which for fixed $n$ lie in $L^{1}(G) \cap L^{2}(G)$. It implies that the trace $\operatorname{Tr}\left[\widehat{\psi}(\sigma, n)^{\dagger} \widehat{\psi}(\sigma, n)\right]$ is integrable with respect to $d \nu(\sigma) d n$; hence it is an integrable function on $\mathcal{N}$ for almost all $\sigma \in \widehat{G}_{r}$. 
This suggests to equip the fibers at fixed $\sigma \in \widehat{G}_{r}$ with the structure of a Hilbert space which we denote by $L_{\sigma \check{\sigma}}^{2}(\mathcal{N})$. We shall also need various subspaces of $L_{\sigma \check{\sigma}}^{2}(\mathcal{N})$ and for convenient reference we collect them in the following definition.

Definition 2.2. The Hilbert spaces

$$
\begin{aligned}
L_{\sigma \check{\sigma}}^{2}(\mathcal{N}) & :=\left\{F: \mathcal{N} \rightarrow \mathcal{L}_{\sigma} \otimes \check{\mathcal{L}}_{\breve{\sigma}} \mid(F, F)_{\sigma \check{\sigma}}<\infty\right\} \cong \mathcal{L}_{\sigma} \otimes \check{\mathcal{L}}_{\check{\sigma}} \otimes L^{2}(\mathcal{N}), \\
L_{\sigma \check{\sigma}}^{2}(\mathcal{N})_{0}: & =\left\{F: \mathcal{N} \rightarrow \mathcal{L}_{\sigma} \otimes \check{\mathcal{L}}_{\check{\sigma}} \mid(F, F)_{\sigma \check{\sigma}}<\infty, F\left(k^{-1} n\right) \pi_{\sigma}(k)^{\dagger}=F(n)\right\},
\end{aligned}
$$

where $\left(F_{1}, F_{2}\right)_{\sigma \check{\sigma}}:=\int d n \operatorname{Tr}\left[F_{1}^{\dagger}(n) F_{2}(n)\right]$ are called the fiber spaces of $L^{2}(G \times \mathcal{N})$ and $L^{2}\left(\mathcal{M}_{r}\right)$, respectively. Let $\widehat{K}$ be the unitary dual of $K, \kappa \in \widehat{K}$, and $V_{\breve{\kappa}} \subset \mathcal{L}_{\check{\sigma}}$ the subspaces in Eq. 2.45) below. Then

$$
\begin{aligned}
L_{\sigma \breve{\kappa}}^{2}(\mathcal{N}) & :=\left\{f: \mathcal{N} \rightarrow \mathcal{L}_{\sigma} \otimes V_{\breve{\kappa}} \mid(f, f)_{\sigma \check{\kappa}}<\infty\right\} \cong \mathcal{L}_{\sigma} \otimes V_{\breve{\kappa}} \otimes L^{2}(\mathcal{N}), \\
L_{\sigma \check{\kappa}}^{2}(\mathcal{N})_{0} & :=\left\{f: \mathcal{N} \rightarrow \mathcal{L}_{\sigma} \otimes V_{\breve{\kappa}} \mid f(k n)=f(n) r_{\kappa}(k)^{\dagger},(f, f)_{\sigma \check{\kappa}}<\infty\right\},
\end{aligned}
$$

where $\left(f_{1}, f_{2}\right)_{\sigma \check{\kappa}}:=\int d n \operatorname{Tr}_{V_{\check{\kappa}}}\left[f_{1}(n)^{\dagger} f_{2}(n)\right]$ are called the $\kappa$-channels of $L_{\sigma \check{\sigma}}^{2}(\mathcal{N})$ and $L_{\sigma \check{\sigma}}^{2}(\mathcal{N})_{0}$, respectively. The adjoints of the singlet channels $\kappa=0$ lead to spaces

$$
\begin{aligned}
L_{\sigma}^{2}(\mathcal{N}) & :=\left\{f: \mathcal{N} \rightarrow \mathcal{L}_{\sigma} \mid \int d n(f(n), f(n))_{\sigma}<\infty\right\} \cong \mathcal{L}_{\sigma} \otimes L^{2}(\mathcal{N}), \\
L_{\sigma}^{2}(\mathcal{N})_{0} & :=\left\{f \in L_{\sigma}^{2}(\mathcal{N}) \mid f(k n)=\pi_{\sigma}(k) f(n)\right\} .
\end{aligned}
$$

With the definition (2.37 a) the Fourier transformation (2.33) becomes an isometry

$$
\begin{aligned}
\mathcal{D} \ni \psi & \longmapsto \widehat{\psi} \in \int^{\oplus} d \nu(\sigma) L_{\sigma \check{\sigma}}^{2}(\mathcal{N}), \\
\int d g d n \psi(g, n)^{*} \psi(g, n) & =\int_{\widehat{G}_{r}} d \nu(\sigma)(\widehat{\psi}, \widehat{\psi})_{\sigma \check{\sigma}}
\end{aligned}
$$

which extends uniquely to an isometry between Hilbert spaces. Since the trace class operators form an ideal in the algebra of all bounded linear operators on $\mathcal{L}_{\sigma}$ for all $\psi \in \mathcal{D}$ the trace $\operatorname{Tr}\left[\pi_{\sigma}(g) \widehat{\psi}(\sigma, n)\right]$ is defined pointwise for all $(g, n) \in G \times \mathcal{N}$ and it is a continuous function in $g$. For the same reason $\left[\rho\left(g_{0}\right) \ell\left(g_{1}\right) \psi\right]^{\wedge}(\sigma, n)=\pi_{\sigma}\left(g_{0}\right) \widehat{\psi}(\sigma, n) \pi_{\sigma}\left(g_{1}\right)^{\dagger}$ is a trace class operator for all $g_{0}, g_{1}$ if $\widehat{\psi}(\sigma, n)$ is. As a consequence $L_{\sigma \check{\sigma}}^{2}(\mathcal{N})$ carries a unitary representation $\pi_{\sigma \check{\sigma}}$ of $G \times G$

$$
\begin{aligned}
& \pi_{\sigma \check{\sigma}}\left(g_{0}, g_{1}\right) F(n):=\pi_{\sigma}\left(g_{0}\right) F(n) \pi_{\sigma}\left(g_{1}\right)^{\dagger}, \\
& \left(\pi_{\sigma \check{\sigma}}\left(g_{0}, g_{1}\right) F_{1}, \pi_{\sigma \check{\sigma}}\left(g_{0}, g_{1}\right) F_{2}\right)_{\sigma \check{\sigma}}=\left(F_{1}, F_{2}\right)_{\sigma \check{\sigma}} .
\end{aligned}
$$

It coincides with $\pi_{\sigma} \times \pi_{\check{\sigma}}$, the outer tensor product of the two representations (which is irreducible [15], Thm 7.20]) whenever both of the factors are. The isometry (2.40) 
therefore also provides the decomposition of $\rho \times \ell$, the outer tensor product of the right and the left regular representation of $G$ into a direct integral of ( $\nu$-almost everwhere) irreducible representations,

$$
\rho \times \ell=\int d \nu(\sigma) \pi_{\sigma} \times \pi_{\check{\sigma}}
$$

With these preparations at hand we can turn to the decomposition of $L^{2}\left(\mathcal{M}_{r}\right)$, which we naturally identified with the subspace of $L^{2}(G \times \mathcal{N})$ consisting of left $K$ invariant functions. Clearly the left $K$ invariance of the function $\psi$ translates into the following condition on the Fourier coefficients

$$
\left[\ell_{r}(k) \psi \hat{\psi}(\sigma, n)=\widehat{\psi}\left(\sigma, k^{-1} n\right) \pi_{\sigma}(k)^{\dagger} \stackrel{!}{=} \widehat{\psi}(\sigma, n) .\right.
$$

We also introduce the corresponding $K$-singlet subspace of $L_{\sigma \check{\sigma}}^{2}(\mathcal{N})$ as in $(2.37 \mathrm{~b})$. Since $\pi_{\sigma}^{\dagger}$ 'acting from the right' is unitarily equivalent to $\pi_{\check{\sigma}}$, in representation theoretical terms (2.43) means

$$
\ell_{\mathcal{N}} \times\left.\pi_{\check{\sigma}}\right|_{K} \stackrel{!}{=} \mathrm{id}
$$

where $\ell_{\mathcal{N}}(k) F(n):=F\left(k^{-1} n\right)$. The condition (!) can be understood as the projection onto the subspace of left $K$ singlets in a decomposition of $L^{2}(G) \otimes L^{2}(\mathcal{N})$ which we prepare now.

First recall that the restriction of $\pi_{\check{\sigma}}$ to the subgroup $K$ decomposes as follows

$$
\left.\pi_{\check{\sigma}}\right|_{K}=\bigoplus_{\widehat{K}_{\check{\sigma}}} m_{\check{\kappa}} r_{\check{\kappa}}, \quad \mathcal{L}_{\check{\sigma}}=\bigoplus_{\check{\kappa}_{\widehat{\kappa}} \in \widehat{K}_{\check{\sigma}}} m_{\check{\kappa}} V_{\check{\kappa}}
$$

Here the subset $\widehat{K}_{\check{\sigma}} \subset \widehat{K}$ for which the irreducible representations $r_{\check{\kappa}}$ on the finite dimensional vector space $V_{\check{\kappa}}$ occurs with nonzero multiplicity $m_{\check{\kappa}}$ is called the $K$ content of $\pi_{\check{\sigma}}$; see appendix A. Often it is convenient to use a basis of $\mathcal{L}_{\breve{\sigma}}$ obtained by concatenation of the bases $e_{\breve{\kappa} s}, s=0, \ldots, m_{\kappa} \operatorname{dim} V_{\check{\kappa}}-1$, of $m_{\check{\kappa}} V_{\check{\kappa}}$. (Here of course for fixed $\check{\kappa}$ the basis vectors $e_{\breve{\kappa} s}, s=0, \ldots, \operatorname{dim} V_{\breve{\kappa}}-1, e_{\breve{\kappa} s}, s=\operatorname{dim} V_{\check{\kappa}}, \ldots, \operatorname{dim} 2 V_{\breve{\kappa}}-1$, etc are likewise orthogonal.) We shall call

$$
e_{\breve{\kappa} s}, \quad s=0, \ldots, m_{\breve{\kappa}} \operatorname{dim} V_{\breve{\kappa}}-1, \quad \check{\kappa} \in \widehat{K}_{\check{\sigma}},
$$

the $K$-adapted basis of $\mathcal{L}_{\check{\sigma}}$. For operators $F \in L_{\sigma \check{\sigma}}^{2}(\mathcal{N})$ the components with respect to an orthonormal basis $\left\{e_{i}, i \in \mathbb{N}\right\}$, and its dual $\left\{\check{e}_{i}=\left(e_{i}, \cdot\right)_{\sigma}, i \in \mathbb{N}\right\}$ are $F_{i j}:=\check{e}_{i}\left(F e_{j}\right)$, so that $F=\sum_{i j} e_{i} F_{i j} \check{e}_{j}$. In the $K$-adapted basis these become $F_{\kappa s, \kappa^{\prime} s^{\prime}}=e_{\breve{\kappa} s}\left(F e_{\kappa^{\prime} s^{\prime}}\right)$.

In view of (2.44) one has to decompose the representation $\rho \times \ell_{K} \times \ell_{\mathcal{N}}$ of $G \times K$ in order to decompose $L^{2}(G \times \mathcal{N}) \cong L^{2}(G) \otimes L^{2}(\mathcal{N})$. Since the group that is represented 
is really only $G \times K$, the second tensor product should be read as an inner one; however for our analysis it is convenient first to regard it as an outer one too: combining (2.42) with (2.45) gives for the first factor

$$
\rho \times\left.\ell\right|_{K}=\int^{\oplus} d \nu(\sigma) \bigoplus_{\kappa \in \widehat{K}_{\check{\sigma}}} \pi_{\sigma} \times m_{\check{\kappa}} r_{\check{\kappa}}
$$

For the second factor we write

$$
\ell_{\mathcal{N}}=\bigoplus_{\kappa} m_{\mathcal{N} \kappa} r_{\kappa}, \quad L^{2}(\mathcal{N})=\bigoplus_{\kappa} m_{\mathcal{N} \kappa} L_{\kappa}^{2}(\mathcal{N})
$$

where $L_{\kappa}^{2}(\mathcal{N})$ is the subspace of functions transforming irreducibly according to $f\left(k^{-1} n\right)=$ $r_{\kappa}(k) f(n)$ and $m_{\mathcal{N} \kappa}$ are some multiplicities. Combining (2.47) and (2.48) results in

$$
L^{2}(G) \otimes L^{2}(\mathcal{N})=\int d \nu(\sigma) \bigoplus_{\kappa, \kappa^{\prime}} \mathcal{L}_{\sigma} \otimes m_{\check{\kappa}} V_{\breve{\kappa}} \otimes m_{\mathcal{N} \kappa^{\prime}} L_{\kappa^{\prime}}^{2}(\mathcal{N})
$$

The Fourier coefficients transform according to

$$
\left(\rho \times \ell_{K} \times \ell_{\mathcal{N}}\right)\left(g, k, k^{\prime}\right) \widehat{\psi}(\sigma, n)=\pi_{\sigma}(g) \widehat{\psi}(\sigma, n)\left(r_{\check{\kappa}} \times r_{\kappa^{\prime}}\right)\left(k, k^{\prime}\right)
$$

It remains to implement the condition (!) in (2.43), (2.44). Since $\psi\left(k^{-1} g, k^{-1} n\right)=$ $\left(\left.\ell\right|_{K} \times \ell_{\mathcal{N}}\right)(k, k) \psi(g, n)$ this amounts to considering now the inner tensor product $\ell_{K} \otimes \ell_{\mathcal{N}}$ and projecting onto the singlet sector. The reduction of $\left.\ell\right|_{K} \otimes \ell_{\mathcal{N}}$ produces in a first step a direct double sum over $\kappa, \kappa^{\prime}$ of terms of the form $r_{\check{\kappa}} \otimes r_{\kappa^{\prime}}$ with multiplicities $m_{\kappa} m_{\mathcal{N} \kappa^{\prime}}$. In the next step we use that $r_{\breve{\kappa}} \otimes r_{\kappa^{\prime}}$ contains the singlet if and only if $\kappa=\kappa^{\prime}$. The latter readily follows from the general result of [4, 5] (described in Appendix A4) on the singlet content of a tensor product of two unitary representations. As a consequence the direct double sum in (2.49) reduces to a single sum and one can check that the condition (!) comes out correctly: $\widehat{\psi}(\sigma, n)\left(r_{\breve{\kappa}} \times r_{\kappa}\right)(k, k)=\widehat{\psi}\left(\sigma, k^{-1} n\right) r_{\kappa}\left(k^{-1}\right)=\widehat{\psi}(\sigma, n)$. Viewed as functions of $n$ alone the Fourier coefficients obey $f\left(k^{-1} n\right)=f(n) r_{\kappa}(k)$ or $f(k n)=f(n) r_{\kappa}(k)^{\dagger}$.

Therefore, in order to go from $L^{2}(G \times \mathcal{N})$ to $L^{2}\left(\mathcal{M}_{r}\right)$ it is useful to consider the subspaces $L_{\sigma \check{\kappa}}^{2}(\mathcal{N})$ of $L_{\sigma \check{\sigma}}^{2}(\mathcal{N})$ and $L_{\sigma \check{\sigma}}^{2}(\mathcal{N})_{0}$ defined in (2.38). Here we interpret the elements of $\mathcal{L}_{\sigma} \otimes V_{\breve{\kappa}}$ as linear maps from $V_{\kappa} \subset \mathcal{L}_{\sigma}$ to $\mathcal{L}_{\sigma}$; the trace in the inner product (2.37) for generic Hilbert-Schmidt operators reduces to a trace on $V_{\kappa}$ for operators with values in $\mathcal{L}_{\sigma} \otimes V_{\check{\kappa}}$. In components

$$
\operatorname{Tr}_{V_{\kappa}}\left[f_{1}(n)^{\dagger} f_{2}(n)\right]=\sum_{i, s} f_{1}(n)_{i \kappa s}^{*} f_{2}(n)_{i \kappa s}, \quad f(n)=\sum_{i, s} e_{i} f(n)_{i \kappa s} \check{e}_{\kappa s}
$$


where $e_{i}, i \in \mathbb{N}$, is a basis on $\mathcal{L}_{\sigma}$ and $\check{e}_{\kappa s}, s=0, \ldots, m_{\kappa} \operatorname{dim} V_{\kappa}-1$, is a basis of linear forms on $m_{\kappa} V_{\kappa}$. The space $L_{\sigma \check{\kappa}}^{2}(\mathcal{N})$ carries a unitary representation $\pi_{\sigma \check{\kappa}}$ of $G \times K$,

$$
\begin{aligned}
& \pi_{\sigma \check{\kappa}}(g, k) f(n):=\pi_{\sigma}(g) f(n) r_{\kappa}(k)^{\dagger}, \\
& \operatorname{Tr}_{V_{\kappa}}\left[\left[\pi_{\sigma \check{\kappa}}(g, k) f_{1}(n)\right]^{\dagger} \pi_{\sigma \check{\kappa}}(g, k) f_{2}(n)\right]=\operatorname{Tr}_{V_{\kappa}}\left[f_{1}(n)^{\dagger} f_{2}(n)\right] .
\end{aligned}
$$

Note that the product $\pi_{\sigma}(g) f(n)$ still transforms nontrivially under the left diagonal action of $K$ according to $\pi_{\sigma}(g) f(n) \mapsto \pi_{\sigma}(k g) f(k n)=\pi_{\sigma}(k)\left[\pi_{\sigma}(g) f(n)\right] r_{\kappa}\left(k^{-1}\right)$. However, introducing $P_{\kappa}$ as the orthogonal projection from $L^{2}(G)$ onto the subspace transforming according to $\kappa$, traces of the form $\operatorname{Tr}_{V_{\kappa}}\left[P_{\kappa} \pi_{\sigma}(g) f(n)\right]$ are invariant under the left diagonal action of $K$.

Finally we arrive at the desired decomposition of $L^{2}\left(\mathcal{M}_{r}\right)$ into a direct integral of irreducible spaces

$$
L^{2}\left(\mathcal{M}_{r}\right)=\int_{\widehat{G}_{r}} d \nu(\sigma) \bigoplus_{\kappa \in \widehat{K}_{\sigma}} m_{\mathcal{M} \check{\kappa}} L_{\sigma \check{\kappa}}^{2}(\mathcal{N})_{0},
$$

for some multiplicities $m_{\mathcal{M} \check{\kappa}}$. The representation $\rho$ itself has been decomposed into a direct integral of primary representations (that is, 15, p.206, ones which are direct sums of identical copies of some irreducible representation). Here $\rho$ acts as the right regular representation of $G$ on the first argument of the functions $L^{2}\left(\mathcal{M}_{r}\right)$, i.e. $\rho\left(g_{0}\right) \psi(g, n)=$ $\psi\left(g g_{0}, n\right)$. The left $K$ invariance of the functions is broken up into the different $\kappa$ 'channels' on the Fourier coefficients; generic left $K$ invariant functions are built from simple ones. Consistency with the initial decomposition (2.40) fixes the multiplicities

$$
m_{\mathcal{M} \kappa}=m_{\kappa}
$$

where $m_{\kappa}$ are the multiplicities occuring in the decomposition of $\left.\pi_{\sigma}\right|_{K}$, see (2.45). Indeed, disregarding the breakup into the irreducible $\kappa$ channels the fiber spaces have the following isometric descriptions

$$
\mathcal{L}_{\sigma}^{2}\left(\mathcal{M}_{r}\right)=\bigoplus_{\kappa \in \widehat{K}_{\sigma}} m_{\mathcal{M} \check{\kappa}} L_{\sigma \check{\kappa}}^{2}(\mathcal{N})_{0} \cong L_{\sigma \check{\sigma}}^{2}(\mathcal{N})_{0} \subset L_{\sigma \check{\sigma}}^{2}(\mathcal{N}) \cong \mathcal{L}_{\sigma} \otimes \bigoplus_{\kappa \in \widehat{K}_{\sigma}} m_{\mathcal{M} \check{\kappa}} V_{\check{\kappa}} \otimes L^{2}(\mathcal{N})
$$

where we used (2.38) in the first isometry and inferred (2.54) from the required match with $L_{\sigma \check{\sigma}}^{2}(\mathcal{N}) \cong \mathcal{L}_{\sigma} \otimes \check{\mathcal{L}}_{\check{\sigma}} \otimes L^{2}(\mathcal{N})$.

The significance of the breakup into the irreducible $\kappa$ channels can be seen more clearly by identifying the pre-images of the functions in $\int d \nu(\sigma) m_{\mathcal{M} \check{\kappa}} L_{\sigma \check{\kappa}}^{2}(\mathcal{N})_{0}$ with respect to the isometry $\psi \mapsto \widehat{\psi}$ on $L^{2}(G \times \mathcal{N})$. Here we take $L_{\sigma \breve{\kappa}}^{2}(\mathcal{N})$ to consist of the zero vector only, if $\kappa \notin \widehat{K}_{\sigma}$. Roughly, the pre-image consists of functions invariant under left convolution with the character $\chi_{\breve{\kappa}}$ of $\check{\kappa} \in \widehat{K}_{\sigma}$. Specifically we set

$$
\begin{aligned}
& \left(E_{\kappa^{\prime}} * \psi\right)(g, n):=d_{\kappa^{\prime}} \int d k \chi_{\kappa^{\prime}}\left(k^{-1}\right) \psi(k g, n), \\
& L_{\kappa}^{2}\left(\mathcal{M}_{r}\right):=\left\{\psi_{\kappa} \in L^{2}\left(\mathcal{M}_{r}\right) \mid E_{\kappa^{\prime}} * \psi_{\kappa}=\delta_{\kappa \kappa^{\prime}} \psi_{\kappa}\right\} .
\end{aligned}
$$


The properties of the functions in this subspace are summarized in the following Lemma.

Lemma 2.1. (a) For $\psi_{\breve{\kappa}} \in L_{\breve{\kappa}}^{2}\left(\mathcal{M}_{r}\right)$, the Fourier coefficients obey

$$
\widehat{\psi_{\breve{\kappa}}} P_{\breve{\kappa}^{\prime}}=\left\{\begin{array}{cl}
0 & \text { for } \kappa^{\prime} \neq \kappa, \\
\widehat{\psi_{\breve{\kappa}}} \in L_{\sigma \breve{\kappa}}^{2}(\mathcal{N})_{0} & \text { for } \kappa^{\prime}=\kappa .
\end{array}\right.
$$

In terms of components with respect to the $K$-adapted basis (2.46) this is equivalent to $\widehat{\psi_{\breve{\kappa}}}(\sigma, n)_{\kappa_{1} s_{1}, \kappa_{2} s_{2}}=f_{\breve{\kappa}}(n)_{\kappa_{1} s_{1}, s_{2}} \delta_{\kappa \kappa_{2}}$, with $f_{\breve{\kappa}} \in L_{\sigma \check{\kappa}}^{2}(\mathcal{N})_{0}$.

(b) For $\psi_{\breve{\kappa}} \in \mathcal{D} \cap L_{\breve{\kappa}}^{2}\left(\mathcal{M}_{r}\right)$ (i.e. $g \mapsto \psi_{\check{\kappa}}(g, n)$ is smooth in $g$ with compact support) we have

$$
\psi_{\check{\kappa}}(g, n)=\int_{\widehat{G}_{r}} d \nu(\sigma) \operatorname{Tr}_{V_{\check{\kappa}}}\left[P_{\breve{\kappa}} \pi_{\sigma}(g) \widehat{\psi_{\check{\kappa}}}(\sigma, n)\right] .
$$

(c) For $\phi_{\breve{\kappa}}, \psi_{\check{\kappa}} \in L_{\check{\kappa}}^{2}\left(\mathcal{M}_{r}\right)$, a Parseval identity holds

$$
\int d g d n \phi_{\breve{\kappa}}(g, n)^{*} \psi_{\breve{\kappa}}(g, n)=\int_{\widehat{G}_{r}} d \nu(\sigma) \int d n \operatorname{Tr}\left[\widehat{\phi}_{\breve{\kappa}}(\sigma, n)^{\dagger} \widehat{\psi}_{\breve{\kappa}}(\sigma, n)\right] .
$$

Proof. (a) We compute $\left(E_{\kappa} * \psi\right)(g, n)$ by inserting the Fourier decomposition (2.33) for $\psi \in L^{2}\left(\mathcal{M}_{r}\right)$. This gives

$$
\begin{aligned}
\left(E_{\kappa} * \psi\right)(g, n) & =\int_{\widehat{G}_{r}} d \nu(\sigma) \operatorname{Tr}\left[\widehat{\psi}(\sigma, n) d_{\kappa} \int_{K} d k \chi_{\kappa}\left(k^{-1}\right) \pi_{\sigma}(k) \pi_{\sigma}(g)\right] . \\
& =\int_{\widehat{G}_{r}} d \nu(\sigma) \operatorname{Tr}_{V_{\kappa}}\left[\pi_{\sigma}(g) \widehat{\psi}(\sigma, n) P_{\kappa}\right] .
\end{aligned}
$$

In the second step we identified the $K$ integral as the projector (A.14) onto the $m_{\kappa} V_{\kappa}$ subspace of $\mathcal{L}_{\sigma}$. This shows $\widehat{\psi}_{\breve{\kappa}} P_{\check{\kappa}^{\prime}}=\delta_{\kappa \kappa^{\prime}} \widehat{\psi}_{\breve{\kappa}}$, or in components with respect to the $K$-adapted basis $\widehat{\psi_{\breve{\kappa}}}(\sigma, n)_{\kappa_{1} s_{1}, \kappa_{2} s_{2}}=f_{\breve{\kappa}}(n)_{\kappa_{1} s_{1}, s_{2}} \delta_{\kappa \kappa_{2}}$, with $f_{\breve{\kappa}} \in L_{\sigma \check{\kappa}}^{2}(\mathcal{N})_{0}$. The equivariance property in the definition of $L_{\sigma \check{\kappa}}^{2}(\mathcal{N})_{0}$ follows from (2.43) and $\widehat{\psi}(\sigma, k n) P_{\breve{\kappa}}=\widehat{\psi}(\sigma, n) \pi_{\sigma}(k)^{\dagger} P_{\breve{\kappa}}=$ $\widehat{\psi}(\sigma, n) P_{\breve{\kappa}} r_{\breve{\kappa}}(k)^{\dagger}$. The fact that $f_{\breve{\kappa}}$ is square integrable in the norm $(,)_{\sigma \breve{\kappa}}$ follows from the Plancherel identity (2.58).

(b) Eq. (2.58) follows from (2.60) and the definition of $L_{\tilde{\kappa}}^{2}\left(\mathcal{M}_{r}\right)_{0}$. Note that the trace is constant on the equivalence classes $(g, n) \sim(k g, k n)$, although the product $\pi_{\sigma}(g) \widehat{\psi_{\breve{\kappa}}}(\sigma, n)$ itself transforms nontrivially under $(g, n) \mapsto(k g, k n)$.

(c) This follows from (a) and (2.36).

We add some comments on Lemma 2.1. First, in view of (2.53) the result can be summarized by stating that the map $\psi_{\breve{\kappa}} \mapsto \widehat{\psi_{\breve{\kappa}}}$ in (2.58) provides a partial isometry

$$
L_{\breve{\kappa}}^{2}\left(\mathcal{M}_{r}\right) \longrightarrow \int_{\widehat{G}_{r}}^{\oplus} d \nu(\sigma) m_{\mathcal{M} \check{\kappa}} L_{\sigma \check{\kappa}}^{2}(\mathcal{N})_{0}
$$


Next we discuss two special cases, where in the decomposition (2.58) only "class 1" representations occur (i.e. representations which contain a vector invariant under the action of the subgroup $K)$. The first case is that of $\rho(K)$ singlets in $L^{2}\left(\mathcal{M}_{r}\right)$. In the decomposition (2.33) functions obeying $\psi(g k, n)=\psi(g, n)$ are characterized by Fourier coefficients with $P_{0} \widehat{\psi}(\sigma, n)=\widehat{\psi}(\sigma, n)$, where $P_{0}$ is the projector onto the singlet sector $\left({ }^{\prime} \kappa=0\right.$ '). In this case Eq. (2.33) reduces to

$$
\begin{aligned}
\psi(g K, n) & =\int_{\widehat{G / K}} d \nu(\sigma) P_{0}\left[\widehat{\psi}(\sigma, n) \pi_{\sigma}(g K)\right] \\
P_{0} \widehat{\psi}(\sigma, n) & =\int_{G / K} d \gamma_{G / K}(g K) \psi(g K, n) P_{0} \pi_{\sigma}\left(K^{-1} g^{-1}\right) .
\end{aligned}
$$

Since the the functions $g \mapsto P_{0} \pi_{\sigma}(g)$ are left $K$-invariant by definition only 'class 1 ' representations (with respect to $K$ ) occur in (2.62). Specifically, consistency with the harmonic analysis on $Q=G / K$ requires that only those (class 1 ) irreducible representions occur which enter the harmonic analysis on $G / K$. We have written $\widehat{G / K} \subset \widehat{G}_{r}$ for this subset; as described in Appendix A.7 it contains the spherical principal series representations only.

For Fourier coefficients obeying in addition (2.43) the projection $P_{0}\left[\widehat{\psi}(\sigma, n) \pi_{\sigma}(g K)\right]$ is invariant under $(g, n) \mapsto(k g, k n)$. In this case the functions in (2.62) define a subspace of $L^{2}\left(\mathcal{M}_{r}\right)$. Note however that it is not $\rho(G)$ irreducible; the latter requires Fourier coefficients satisfying $P_{0} \widehat{\psi}(\sigma, n) P_{\kappa}=P_{0} \widehat{\psi}(\sigma, n)$ for some $\kappa \in \widehat{K}$. To proceed recall that the section $g_{s}: Q \rightarrow G$ provides an injective imbedding of $Q$ in $G$. We set

$$
\begin{aligned}
& E_{\sigma, \check{\kappa}}(q):=P_{\breve{\kappa}} \pi_{\sigma}\left(g_{s}(q)\right) P_{0}, \quad E_{\sigma, \check{\kappa}}(q)^{\dagger}=P_{0} \pi_{\sigma}\left(g_{s}(q)^{-1}\right) P_{\breve{\kappa}}, \\
& \psi_{\breve{\kappa}}(q, n):=\psi_{\breve{\kappa}}\left(g_{s}(q)^{-1}, n\right),
\end{aligned}
$$

in terms of which the expansion (2.58) takes the form

$$
\begin{aligned}
& \psi_{\breve{\kappa}}(q, n)=\int_{\widehat{G / K}} d \nu(\sigma) \operatorname{Tr}\left[E_{\sigma, \breve{\kappa}}(q) \widehat{\psi_{\breve{\kappa}}}(\sigma, n)\right], \\
& \widehat{\psi_{\breve{\kappa}}}(\sigma, n)=\int_{Q} d \gamma_{Q}(q) \psi_{\breve{\kappa}}(q, n) E_{\sigma, \breve{\kappa}}(q)^{\dagger} .
\end{aligned}
$$

Another subspace of $L^{2}\left(\mathcal{M}_{r}\right)$ in whose decomposition only class 1 irreducible representations appear is the singlet $(\kappa=0)$ sector of (2.53). On account of Lemma 2.1 this sector arises from functions $\psi_{0} \in L_{0}\left(\mathcal{M}_{r}\right)$, that is, functions obeying $\psi_{0}\left(g, k^{-1} n\right)=\psi_{0}(g, n)=$ $\psi_{0}(k g, n)$. The Fourier decomposition takes the form

$$
\begin{aligned}
& \psi_{0}(g, n)=\int_{\widehat{G / K}} d \nu(\sigma) P_{0}\left[\pi_{\sigma}(g) \widehat{\psi_{0}}(\sigma, n)\right] \\
& \widehat{\psi_{0}}(\sigma, n)=\widehat{\psi_{0}}(\sigma, n) P_{0}=\int d g \psi_{0}(g, n) \pi_{\sigma}\left(g^{-1}\right) P_{0} .
\end{aligned}
$$


We can now summarize the results on the group theoretical decomposition. Since the action of $\rho(G)$ on $L^{2}\left(\mathcal{M}_{r}\right)$ corresponds to the original $\ell_{\mathcal{M}}$ action of $G$ on $L^{2}(\mathcal{M})$ (not to be confused with the left regular representation of $G$ on itself denoted by $\ell$ ) we have found the desired group theoretical decomposition (2.10): only a subset of $\widehat{G}$, the restricted dual $\widehat{G}_{r}$ appears, and each $\sigma \in \widehat{G}_{r}$ occurs with infinite multiplicity $\operatorname{dim}\left(\mathcal{L}_{\sigma}\right)$. The measure $d \mu_{\ell_{\mathcal{M}}}$ is the Plancherel measure on $\widehat{G}_{r}$ and the copies are counted with the counting measure on $\widehat{K}$. We continue to use the realization $L^{2}\left(\mathcal{M}_{r}\right)$, where the group acts via the right regular representation on the first argument of the functions $\psi=\psi_{r}$. For convenient reference we collect the results in:

Proposition 2.2. (a) The Hilbert space $L^{2}\left(\mathcal{M}_{r}\right)$ decomposes under the action of the unitary representation $\rho(G)$ according to

$$
L^{2}\left(\mathcal{M}_{r}\right)=\int_{\widehat{G}_{r}} d \nu(\sigma) \bigoplus_{\kappa \in \widehat{K}_{\sigma}} m_{\check{\kappa}} L_{\sigma \check{\kappa}}^{2}(\mathcal{N})_{0}=: \int_{\widehat{G}_{r}} d \nu(\sigma) \mathcal{L}_{\sigma}^{2}\left(\mathcal{M}_{r}\right)
$$

with $L_{\sigma, \check{\kappa}}^{2}(\mathcal{N})_{0}$ defined in (2.38), and $m_{\kappa}$ the multiplicities in 2.45).

(b) Disregarding the breakup into irreducible $\kappa$ channels the fiber spaces have the following descriptions

$$
\mathcal{L}_{\sigma}^{2}\left(\mathcal{M}_{r}\right)=\bigoplus_{\kappa \in \widehat{K}_{\sigma}} m_{\check{\kappa}} L_{\sigma \check{\kappa}}^{2}(\mathcal{N})_{0} \cong L_{\sigma \check{\sigma}}^{2}(\mathcal{N})_{0} \subset L_{\sigma \check{\sigma}}^{2}(\mathcal{N}) \cong \mathcal{L}_{\sigma} \otimes \check{\mathcal{L}}_{\check{\sigma}} \otimes L^{2}(\mathcal{N})
$$

with $L_{\sigma \check{\sigma}}^{2}(\mathcal{N})$ defined in 2.37$)$.

(c) On the subspace of $L^{2}\left(\mathcal{M}_{r}\right)$ containing a $\rho(K)$ singlet the decomposition has support only on the spherical principal series representations.

The content of Proposition 2.2 can be illustrated by matching (2.66) against a direct decomposition of $L^{2}(\mathcal{M}) \simeq L^{2}(Q)^{\otimes \nu}$ into irreducibles. From (A.26) one has

$$
L^{2}(Q)^{\otimes \nu} \simeq \int \frac{d \omega_{1}}{\left|c\left(\omega_{1}\right)\right|^{2}} \cdots \frac{d \omega_{1}}{\left|c\left(\omega_{1}\right)\right|^{2}} \mathcal{L}_{\omega_{1}} \otimes \ldots \otimes \mathcal{L}_{\omega_{\nu}}
$$

where $\mathcal{L}_{\omega}, \omega \in \widehat{Q} \subset \mathbb{R}^{\operatorname{dim} A}$ carries the spherical principal series represenation $\pi_{\omega, 0}$. The decomposition problem essentially amounts to decomposing arbitary tensor products $\mathcal{L}_{\omega_{1}} \otimes \ldots \otimes \mathcal{L}_{\omega_{\nu}}$ of spherical principal series representations. This can be done inductively by first decomposing $\mathcal{L}_{\omega_{1}} \otimes \mathcal{L}_{\omega_{2}}=\int d \mu\left(\omega_{1}, \omega_{2} \mid \sigma\right) \mathcal{L}_{\sigma}$, then $\mathcal{L}_{\sigma} \otimes \mathcal{L}_{\omega_{3}}$ for any $\sigma \in \widehat{G}$ in the support of the measure $d \mu\left(\omega_{1}, \omega_{2} \mid \sigma\right)$, and so on. Depending on what is known about the support of $d \mu\left(\omega_{1}, \omega_{2} \mid \sigma\right)$ in $\widehat{G}$ this strategy requires knowledge of large portions of the complete branching rules. Since the complete branching rules are known only for a few noncompact groups (like $\mathrm{SL}(2, \mathbb{R})$, see 36]) this would be tedious, to say the least. 
Information about the support of the measure $d \mu\left(\omega_{1}, \omega_{2} \mid \sigma\right)$, seems to be available only in a few cases, like $\mathrm{SO}_{0}(1, N)$ from [11]. According to Theorem 10.5 given there, a tensor product of two spherical principal series unitary irreducible representations (UIR) of $\mathrm{SO}_{0}(1, N)$ decomposes into principal series UIR only, for $N \geq 4$ even. (For $N$ odd this holds trivially and for $N=2$ it is manifestly not true, see [36]). For more than two tensor copies (or for $N=2$ ), however, the result is insufficient to exclude the occurrence of UIR other than principal series representations. For $\nu=3$ one would need to know how the tensor product of a spherical and a generic (non-spherical) principal series decomposes. This might include discrete series representations and possibly others. So, for the direct decomposition of $\nu \geq 4$ fold tensor products of the spherical principal series, one needs to know essentially the complete branching rules.

As a spin-off of Proposition 2.2 we have

Corollary 2.3. (a) Let $\mathcal{L}_{\omega_{1}} \otimes \ldots \otimes \mathcal{L}_{\omega_{\nu}}$ be an arbitrary tensor products of spherical principal series representations. Then for almost all $\omega_{1}, \ldots, \omega_{\nu}$ with respect to the measure in (2.68) its decomposition does not contain the singlet (as the only finite dimensional UIR). (b) Let $Q\left(\omega_{1}, \ldots, \omega_{\nu} \mid \sigma\right)\left(q_{1}, \ldots, q_{\nu}\right)$ be an intertwiner from $\mathcal{L}_{\omega_{1}} \otimes \ldots \otimes \mathcal{L}_{\omega_{\nu}}$ to $\mathcal{L}_{\sigma}$, $\sigma \in \widehat{G}$. Then, for almost all $\omega_{1}, \ldots, \omega_{\nu}$

$$
\int_{K} d k Q\left(\omega_{1}, \ldots, \omega_{\nu} \mid \sigma\right)\left(k q_{1}, \ldots, k q_{\nu}\right)=0
$$

unless $\sigma$ is again a spherical principal series representation.

Part (a) generalizes a result by Fulling [16] for tensor products of spherical principal representations to all reductive linear Lie groups. Part (b) provides a nontrivial 'sumrule' whenever explicit expressions for the interwiners are available. The 'almost all' caveat is needed because the Harish-Chandra c-function defining the measure in (2.68) is a meromorphic function over the complexification of $\widehat{Q}$ (viz $a_{\mathbb{C}}^{*}$, where $a$ is the Lie algebra of the subgroup A in the Iwasawa decomposition.) See e.g. [24], Chapter, II.3. At the position of a pole the decomposition (2.68) yields no information about the fiber spaces, but otherwise all spherical principal series representations occur 24], Chapter VI.3. The location of the poles of $c$ can be analyzed from the explicit expressions [24], which could be used to specify the exceptional sets.

\section{The spectral problem on the invariant fibers}

We proceed to study the consequences of the orbit decomposition for selfadjoint operators commuting with the group action. It is natural to consider the same class of integral operators as in Definition 2.1, but drop the positivity requirements. 
Definition 3.1. A standard invariant selfadjoint operator $\mathbf{A}$ on $L^{2}(\mathcal{M})$ is an integral operator with a symmetric continuous kernel $A: \mathcal{M} \times \mathcal{M} \rightarrow \mathbb{C}$, which obeys (3.1) below and satisfies $A\left(d(g) m, d(g) m^{\prime}\right)=A\left(m, m^{\prime}\right)$ for all $g \in G, m, m^{\prime} \in \mathcal{M}$. Its image $\Phi^{-1} \circ \mathbf{A} \circ \Phi$ under the isometry in (2.32) is also denoted by $\mathbf{A}$ and is an integral operator on $L^{2}\left(\mathcal{M}_{r}\right)$ with kernel $\mathcal{A}: G \times \mathcal{N} \times \mathcal{N} \rightarrow \mathbb{C}$, subject to 3.4) below and (3.1). Here

$$
\sup _{m} \int d m^{\prime} \mid A\left(m, m^{\prime}\left|=\sup _{n} \int d n^{\prime} d g\right| \mathcal{A}\left(g, n, n^{\prime}\right) \mid=: \quad K_{\mathcal{A}}<\infty .\right.
$$

As in Definition 2.1 the condition (3.1) entails that $\mathbf{A}$ is well-defined as a bounded selfadjoint operator from $L^{p}$ to $L^{p}$ for all $1 \leq p \leq \infty$. The structure of the kernels $A$ and $\mathcal{A}$ of course implies that the operators A commute with $\ell_{\mathcal{M}}(G)$ and $\rho(G)$, respectively.

\subsection{Basic consequences of the $\rho(G)$ invariance}

As a first result we have:

Proposition 3.1. (a) Every standard invariant selfadjoint operator $\mathbf{A}$ on $L^{2}(\mathcal{M})$ has purely essential spectrum, $\operatorname{Spec}(\mathbf{A})=$ ess-Spec $(\mathbf{A})$.

(b) A transfer operator commuting with $\ell_{\mathcal{M}}(G)$ cannot have normalizable ground states; hence $\|\mathbf{T}\| \in \operatorname{c-Spec}(\mathbf{T})$, or $\|\mathbf{T}\|$ is a limit point of eigenvalues of infinite multiplicity.

Proof. (a) Recall from Eqs. (2.10) and (2.11) that the measure $\mu_{\ell_{\mathcal{M}}}$ on $\widehat{G}$ defining the decomposition of the unitary representation $\ell_{\mathcal{M}}$ is related to that defining the generalized

eigenspaces of $\mathbf{A}$ by $\int_{\operatorname{Spec}(\mathbf{A})} d \mu(\lambda, \sigma)=d \mu_{\ell_{\mathcal{M}}}(\sigma)$. By Proposition 2.2 the measure $\mu_{\ell_{\mathcal{M}}}(\sigma)$ can be identified with the Plancherel measure. For the noncompact Lie groups considered the support of the Plancherel measure contains only infinite dimensional representations (see Appendix A). Therefore, whenever a generalized eigenspace $\mathcal{E}_{\lambda, \sigma}(\mathbf{A})$ occurs in (2.4) its spectral value $\lambda$ has infinite multiplicity. This excludes that $\lambda$ lies in the discrete spectrum of the operator; hence $\lambda \in \operatorname{ess-Spec}(\mathbf{A})$. Note that it is not excluded that A has point spectrum of infinite multiplicity, i.e. infinite multiplets of normalizable eigenfunctions for some spectral value $\lambda$.

(b) Let now $\mathbf{T}$ be a transfer operator on $L^{2}(\mathcal{M})$ commuting with $\ell_{\mathcal{M}}(G)$. Assume it has a normalizable ground state, i.e. a solution of $\mathbf{T} \psi=\|\mathbf{T}\| \psi$, with $\psi \in L^{2}(\mathcal{M})$. By a well known result, based on the Perron-Frobenius theorem (see for instance [19, 44]), this ground state would be unique and therefore invariant under the action of $\ell_{\mathcal{M}}(G)$, i.e. an $\ell_{\mathcal{M}}$ singlet. This entails that $(\psi, \psi)$ diverges as the infinite volume of the group is 'overcounted'. Thus $\|\mathbf{T}\|$ cannot be a eigenvalue. By part (a) it can also not be a limit point of the discrete spectrum (since there is none), leaving only the possibilities: $\|\mathbf{T}\| \in \mathrm{c}-\operatorname{Spec}(\mathbf{T})$ or $\|\mathbf{T}\|$ a limit point of eigenvalues of infinite multiplicity. 
To proceed, let us momentarily denote the selfadjoint operator $\mathbf{A}$ in the realization acting on $L^{2}\left(\mathcal{M}_{r}\right)$ by $\mathbf{A}_{r}:=\Phi^{-1} \circ \mathbf{A} \circ \Phi$, with $\Phi$ as in (2.32). We write $A_{r}\left(g, n, g^{\prime}, n^{\prime} ; t\right)$ for the corresponding kernel. Writing out $\left(\Phi^{-1} \circ \mathbf{A} \circ \Phi \psi_{r}\right)(g, n)$ one finds

$$
A_{r}\left(g, n, g^{\prime}, n^{\prime}\right)=A\left(\phi^{-1}(g, n), \phi^{-1}\left(g^{\prime}, n^{\prime}\right)\right)=A\left(g^{\prime} g^{-1} q^{\uparrow}, g^{\prime} g^{-1} n, q^{\uparrow}, n^{\prime}\right)
$$

In the last step we used the invariance of $\mathbf{A}$, i.e. $\ell_{\mathcal{M}}(g) \circ \mathbf{A}=\mathbf{A} \circ \ell_{\mathcal{M}}(g)$ for all $g \in G$. Since $\mathbf{A}_{r}^{t}=\Phi^{-1} \circ \mathbf{A}^{t} \circ \Phi$ for $t \in \mathbb{N}$, also all iterated kernels will be related by (3.2).

For the reasons explained in Section 2.3 the kernel $A_{r}$ is most useful. From the last expression in (3.2) one sees explicitly that $A_{r}$ only depends on the right invariant combination $g^{\prime} g^{-1}$, consistent with $\rho(g) \circ \mathbf{A}_{r}=\mathbf{A}_{r} \circ \rho(g)$ for the operator. Further $A_{r}$ is manifestly constant on the left equivalence classes $(k g, k n) \sim(g, n)$ and $\left(k^{\prime} g^{\prime}, k^{\prime} n^{\prime}\right) \sim\left(g^{\prime}, n^{\prime}\right)$ with $\left(k, k^{\prime}\right) \in K \times K$. We set

$$
\mathcal{A}\left(g g^{\prime-1}, n, n^{\prime} ; t\right):=A_{r}\left(g, n, g^{\prime}, n^{\prime} ; t\right)
$$

and note

$$
\begin{aligned}
& \mathcal{A}\left(g, n, n^{\prime}\right)=\mathcal{A}\left(g^{-1}, n^{\prime}, n\right), \\
& \mathcal{A}\left(k g, k n, n^{\prime} ; t\right)=\mathcal{A}\left(g, n, n^{\prime} ; t\right)=\mathcal{A}\left(g k^{-1}, n, k n^{\prime} ; t\right), \quad k \in K .
\end{aligned}
$$

We may thus interpret $\mathbf{A}_{r}$ either as an integral operator acting on $L^{2}\left(\mathcal{M}_{r}\right)$ or on $L^{2}(G \times \mathcal{N})$; in the latter interpretation it automatically projects onto the $\ell_{r}(K)$ invariant subspace (with $\ell_{r}$ defined in (2.31)). This means that the nonzero spectrum of A lies automatically in that subspace. For convenience, we will therefore work with this interpretation of $\mathbf{A}_{r}$.

To simplify the notation we drop the subscript $r$ in the following and write $\mathbf{A}$ for $\mathbf{A}_{r}$ etc. Due to the properties (3.3), (3.4) A respects the fiber decomposition (2.66) in the following sense:

Lemma 3.1. Let $\Sigma$ be a measurable subset of $\widehat{G}_{r}, \Sigma^{c}$ its complement and let $\mathcal{H}$ and $\mathcal{H}^{c}$ be the corresponding subspaces of $L^{2}(G)$ (which are orthogonal complements of each other):

$$
\begin{aligned}
\mathcal{H} & =\int_{\Sigma}^{\oplus} d \nu(\sigma) \mathcal{L}_{\sigma} \otimes \check{\mathcal{L}}_{\check{\sigma}} \otimes L^{2}(\mathcal{N}), \\
\mathcal{H}^{c} & =\int_{\Sigma^{c}}^{\oplus} d \nu(\sigma) \mathcal{L}_{\sigma} \otimes \check{\mathcal{L}}_{\check{\sigma}} \otimes L^{2}(\mathcal{N}) .
\end{aligned}
$$

Then $\mathbf{A} \mathcal{H} \subset \mathcal{H}$ and $\mathbf{A} \mathcal{H}^{c} \subset \mathcal{H}^{c}$. 
Proof. Let $\psi \in \mathcal{H}, \psi_{c} \in \mathcal{H}^{c}$ and consider

$$
s(g):=\left(\psi_{c}, \mathbf{A} \rho(g) \psi\right)=\left(\rho(g)^{-1} \psi_{c}, \mathbf{A} \psi\right) .
$$

The first expression shows that the Fourier transform $\widehat{s}$ of $s$ is supported in $\Sigma$. On the other hand, the second expression shows that the Fourier transform is supported in $\Sigma^{c}$. This is possible only if $s$ vanishes identically. Putting $g=e$, the lemma follows.

\subsection{Fiber decomposition of invariant selfadjoint operators}

In view of Lemma 3.1 the operator $\mathbf{A}$ should also map the fiber spaces $\mathcal{L}_{\sigma}^{2}\left(\mathcal{M}_{r}\right)$ in (2.67) onto itself. To give precise meaning to this statement we have to construct fiber operators $\mathbf{A}(\sigma)$ from $\mathbf{A}$. In a first step one applies the expansion (2.33) to the kernel $\mathcal{A}\left(g, n, n^{\prime}\right)$

$$
\begin{aligned}
& \mathcal{A}\left(g, n, n^{\prime}\right)=\int_{\widehat{G}_{r}} d \nu(\sigma) \operatorname{Tr}\left[\widehat{\mathcal{A}}\left(\sigma, n^{\prime}, n\right) \pi_{\sigma}(g)\right] . \\
& \widehat{\mathcal{A}}\left(\sigma, n^{\prime}, n\right):=\int_{G} d g \mathcal{A}\left(g, n, n^{\prime}\right) \pi_{\sigma}\left(g^{-1}\right) .
\end{aligned}
$$

The swapped order in the $\left(n, n^{\prime}\right)$ arguments was chosen in order to have

$$
\widehat{\mathcal{A}}\left(\sigma, k^{\prime} n^{\prime}, k^{-1} n\right)_{\kappa_{1} s_{1}, \kappa_{2} s_{2}}=\sum_{s s^{\prime}} r_{\kappa_{1}}\left(k^{\prime}\right)_{s_{1} s} \widehat{\mathcal{A}}\left(\sigma, n^{\prime}, n\right)_{\kappa_{1} s, \kappa_{2} s^{\prime}} r_{\kappa_{2}}(k)_{s^{\prime} s_{2}}
$$

where the indices $\kappa, s$ etc. refer to the $K$-adapted basis (2.46). Note further the hermiticity and diagonal $K$ invariance imply

$$
\widehat{\mathcal{A}}\left(\sigma, n^{\prime}, n\right)=\widehat{\mathcal{A}}\left(\sigma, n, n^{\prime}\right)^{\dagger}, \quad \operatorname{Tr}_{V_{\kappa}} \widehat{\mathcal{A}}\left(\sigma, k n^{\prime}, k n\right)=\operatorname{Tr}_{V_{\kappa}} \widehat{\mathcal{A}}\left(\sigma, n^{\prime}, n\right)
$$

Since by (3.1) for a.e. $n, n^{\prime}$ the kernel $\mathcal{A}$ is in $L^{1}(G)$, its Fourier transform $\widehat{\mathcal{A}}\left(\sigma, n, n^{\prime}\right)$ is a compact operator on $\mathcal{L}_{\sigma}$ for a.e. $n, n^{\prime}$, see [15], Theorem 7.6. Moreover (3.1) implies the following bound on $\widehat{\mathcal{A}}\left(\sigma, n, n^{\prime}\right)$

$$
\sup _{n} \int d n^{\prime}\left\|\widehat{\mathcal{A}}\left(\sigma, n, n^{\prime}\right)\right\| \leq K_{A}
$$

where $\|\cdot\|$ denotes the operator norm on $\mathcal{L}_{\sigma}$. If we momentarily introduce the spaces

$$
L_{\sigma}^{p}(\mathcal{N}):=\left\{\psi: \mathcal{N} \rightarrow \mathcal{L}_{\sigma} \mid \int d n(\psi(n), \psi(n))_{\sigma}^{p / 2}<\infty\right\}
$$


it follows first that $\widehat{\mathcal{A}}\left(\sigma, n, n^{\prime}\right)$ defines a bounded linear operator from $L_{\sigma}^{1}(\mathcal{N})$ to $L_{\sigma}^{1}(\mathcal{N})$ as well as from $L_{\sigma}^{\infty}(\mathcal{N})$ to $L_{\sigma}^{\infty}(\mathcal{N})$; by complex interpolation it follows then essentially as in [30] p.173 ff that $\widehat{\mathcal{A}}\left(\sigma, n, n^{\prime}\right)$ defines a bounded linear operator from $L_{\sigma}^{p}(\mathcal{N})$ to $L_{\sigma}^{p}(\mathcal{N})$ for all $p \in[1, \infty]$, where the $p=\infty$ norm is given by $\sup _{n} \sqrt{(\psi(n), \psi(n))_{\sigma}}$.

Furthermore the projection property of $\mathbf{A}$ onto the $\ell_{r}(K)$ invariant subspace translates into an equivariance property analogous to (2.43):

$$
\widehat{\mathcal{A}}\left(\sigma, n^{\prime}, k^{-1} n\right) \pi_{\sigma}\left(k^{-1}\right)=\widehat{\mathcal{A}}\left(\sigma, n^{\prime}, n\right)=\pi_{\sigma}(k) \widehat{\mathcal{A}}\left(\sigma, k^{-1} n^{\prime}, n\right), \quad \text { for } \quad k \in K
$$

using (2.34), (3.4), (3.7) . In the following we wish to convert the action of $\mathbf{A}$ on $L^{2}\left(\mathcal{M}_{r}\right)$ into an action $\mathbf{A}(\sigma)$ on the fibers $\int^{\oplus} d \nu(\sigma) L_{\sigma}^{2}\left(\mathcal{M}_{r}\right)$ such that $\widehat{\mathbf{A} \psi}(\sigma, n)=\mathbf{A}(\sigma) \widehat{\psi}(\sigma, n)$ holds. To this end we note

$$
(\mathbf{A} \psi)(g, n)=\int d g^{\prime} d n^{\prime} \mathcal{A}\left(g g^{\prime-1}, n, n^{\prime}\right) \psi\left(g^{\prime}, n^{\prime}\right)
$$

From the definitions (2.33) one readily computes

$$
\widehat{\mathbf{A} \psi}(\sigma, n)=\int d n^{\prime} \widehat{\psi}\left(\sigma, n^{\prime}\right) \widehat{\mathcal{A}}\left(\sigma, n^{\prime}, n\right)
$$

Note that $\widehat{\mathcal{A}}$ acts from the right on $\widehat{\psi}$; it acts only on the factor $\check{\mathcal{L}}_{\check{\sigma}} \otimes L^{2}(\mathcal{N})$. If one views $\widehat{\psi}$ as a matrix, the 'column' index is unaffected.

We can now define the fiber operator $\mathbf{A}(\sigma)$ by this action from the right, Eq. (3.14), on the elements of $L_{\sigma \check{\sigma}}^{2}(\mathcal{N})$ introduced in (2.37):

$$
\mathbf{A}(\sigma) \widehat{\psi}(\sigma, n):=\widehat{\mathbf{A} \psi}(\sigma, n)
$$

the fiber operators $\mathbf{A}(\sigma)$ then form a measurable field of operators such that

$$
\mathbf{A}=\int_{\widehat{G}_{r}}^{\oplus} d \nu(\sigma) \mathbf{A}(\sigma)
$$

The spectrum of $\mathbf{A}(\sigma)$ has infinite multiplicity because of the spectator role of the first tensor factor: we have

$$
\mathbf{A}(\sigma)=\mathbb{I} \otimes \check{\mathbf{A}}_{\check{\sigma}}
$$

where $\check{\mathbf{A}}_{\check{\sigma}}$ acts on 'from the right'. This means that $\check{\mathbf{A}}_{\check{\sigma}}$ maps the space of bounded linear forms on $\mathcal{L}_{\sigma}$ with values in $L^{2}(\mathcal{N})$ into itself, or equivalently, it is a map from $\check{\mathcal{L}}_{\check{\sigma}} \otimes L^{2}(\mathcal{N})$ into itself. Thus $\check{\mathbf{A}}_{\check{\sigma}}$ is the (partial) dual of an operator $\mathbf{A}_{\sigma}$ mapping $\mathcal{L}_{\sigma} \otimes L^{2}(\mathcal{N})$ into 
itself. We will see that in many cases the spectrum of $\mathbf{A}_{\sigma}$ has only finite multiplicity. As will become clear below, however, both $\mathbf{A}(\sigma)$ and $\mathbf{A}_{\sigma}$ in general mix the different $\kappa$ channels in the decomposition (2.53).

Since $\mathbf{A}$ is a bounded operator, it follows from general considerations (see [15] (7.30)) that $\mathbf{A}(\sigma): L_{\sigma \check{\sigma}}^{2}(\mathcal{N}) \rightarrow L_{\sigma \check{\sigma}}^{2}(\mathcal{N})$ is a bounded operator for almost all $\sigma$ (see also Proposition 3.4 below). Similarly $\mathbf{A}_{\sigma}$ is a bounded operator $L_{\sigma}^{2}(\mathcal{N}) \rightarrow L_{\sigma}^{2}(\mathcal{N})$ for almost all $\sigma$, with $L_{\sigma}^{2}(\mathcal{N})$ as in (2.39a) of Definition 2.2. On this space the left action $\ell_{r}(K)$ corresponds to $f(n) \mapsto \pi_{\sigma}(k) f\left(k^{-1}\right)$; the singlet subspace of $L_{\sigma}^{2}(\mathcal{N})$ under this action is just $L_{\sigma}^{2}(\mathcal{N})_{0}$ as defined in (2.39)

Whenever $\mathbf{A}(\sigma)$ and hence $\mathbf{A}_{\sigma}$ are bounded operators we call $\sigma \in \widehat{G}_{r}$ nonexceptional. This is the case for all $\sigma$ in the discrete part $\widehat{G}_{d}$ of the restricted dual (see Proposition 3.4 below) and for almost all $\sigma$ in the remainder $\widehat{G}_{r} \backslash \widehat{G}_{d}$. Whenever $\sigma$ does not occur as an integration variable we will assume it to be non-exceptional.

Let us now describe the operator $\mathbf{A}_{\sigma}$ explicitly. In terms of the operator valued kernel $\widehat{\mathcal{A}}$, its action is given by

$$
\left(\mathbf{A}_{\sigma} f\right)(n)=\int d n^{\prime} \widehat{\mathcal{A}}\left(\sigma, n^{\prime}, n\right)^{\dagger} f\left(n^{\prime}\right)=\int d n^{\prime} \widehat{\mathcal{A}}\left(\sigma, n, n^{\prime}\right) f\left(n^{\prime}\right)
$$

The equivariance property carries over to $\mathbf{A}_{\sigma}$ :

$$
\left(\mathbf{A}_{\sigma} f\right)(k n)=\pi_{\sigma}(k)\left(\mathbf{A}_{\sigma} f\right)(n)
$$

or, in the $K$-adapted basis $\left(\mathbf{A}_{\sigma} f\right)(k n)_{\kappa s}=\sum_{s} r_{\kappa}(k)_{s s^{\prime}}\left(\mathbf{A}_{\sigma} f\right)(n)_{\kappa s^{\prime}}$. This is true whether or not $f$ satisfies the corresponding equivariance property; it is a reflection of the fact that A projects onto the $\ell_{r}(K)$ invariant subspace of $L^{2}(G \times \mathcal{N})$.

Proposition 3.4. Let A be a standard invariant selfadjoint operator on $L^{2}\left(\mathcal{M}_{r}\right)$ in the sense of Definition 3.1. Then:

(a) The operator $\mathbf{A}$ has a decomposition $\mathbf{A}=\int^{\oplus} d \nu(\sigma)\left(\mathbb{1} \otimes \check{\mathbf{A}}_{\check{\sigma}}\right)$, respecting the fibers in Proposition 2.2. Further $\mathbf{A}_{\sigma}$ is well-defined as a bounded linear and selfadjoint operator on $L_{\sigma}^{2}(\mathcal{N})$ for all $\sigma \in \widehat{G}_{d}$ and almost all $\sigma \in \widehat{G}_{r} \backslash \widehat{G}_{d}$ (called non-exceptional). The associated norms satisfy

$$
\operatorname{ess} \sup _{\sigma}\left\|\mathbf{A}_{\sigma}\right\|=\|\mathbf{A}\|
$$

(b) $\lambda \in$ ess-Spec $(\mathbf{A})=\operatorname{Spec}(\mathbf{A})$ iff for all $\epsilon>0$ the set $\left\{\sigma \in \widehat{G}_{r} \mid \operatorname{Spec}(\mathbf{A}(\sigma)) \cap(\lambda-\epsilon, \lambda+\right.$ $\epsilon) \neq \emptyset\}$ has positive measure, where $\mathbf{A}(\sigma)=\mathbb{I} \otimes \check{\mathbf{A}}_{\sigma}$. Further $\lambda$ is an eigenvalue of $\mathbf{A}$ iff the set $\left\{\sigma \in \widehat{G}_{r} \mid \lambda\right.$ is an eigenvalue of $\left.\mathbf{A}(\sigma)\right\}$ has positive measure. 
Proof. (a) The decomposition has been just explained; the boundedness a.e. as well as (3.20) follow from the general theory of direct integral decompositions of operators (see [15] or [4], Theorem XIII.83). For the sake of completeness we sketch the argument:

For all $\psi \in L^{2}\left(\mathcal{M}_{r}\right)$ one has from (2.33) and (3.15)

$$
(\mathbf{A} \psi)(g, n)=\int_{\widehat{G}_{r}} d \nu(\sigma) \operatorname{Tr}\left[\mathbf{A}(\sigma) \widehat{\psi}(\sigma, n) \pi_{\sigma}(g)\right]
$$

The norm of $\mathbf{A} \psi$ is related to that of $\widehat{\mathbf{A} \psi}(\sigma, n)$ by the Parseval identity (2.36)

$$
(\mathbf{A} \psi, \mathbf{A} \psi)=\int_{\widehat{G}_{r}} d \nu(\sigma)(\mathbf{A}(\sigma) \widehat{\psi}(\sigma), \mathbf{A}(\sigma) \widehat{\psi}(\sigma))_{\sigma \check{\sigma}}
$$

with $(,)_{\sigma \check{\sigma}}$ defined in (2.37). Since $\mathbf{A}$ is a bounded operator the left hand side of $(3.22)$ is finite, hence the integrand on the right hand side is finite $\nu$-almost everywhere. The bound (3.20) follows by choosing sequences of $\psi$ such that their Fourier transforms $\widehat{\psi}(\sigma, n)$ are becoming concentrated at a particular value $\sigma$ (see 15, Proposition 7.33). Further $\mathbf{A}(\sigma)$ is symmetric and has a unique selfadjoint extension for a.e. $\sigma$, which we denote by the same symbol. Since $\mathbf{A}(\sigma)=\mathbb{1} \otimes \check{\mathbf{A}}_{\check{\sigma}}$, the same holds for the fiber operators $\mathbf{A}_{\sigma}$.

(b) The statements about the spectrum in general as well as the eigenvalues follow as in Theorem XIII.85 of [44.

From our analysis we have obtained the following: The Hilbert space $L^{2}\left(\mathcal{M}_{r}\right)$ is resolved into the direct integral $\int^{\oplus} d \nu(\sigma) \mathcal{L}_{\sigma} \otimes \check{\mathcal{L}}_{\check{\sigma}} \otimes L^{2}(\mathcal{N})$. Likewise $\mathbf{A}$ can be resolved into a direct integral $\int^{\oplus} \mathbf{A}(\sigma) d \nu(\sigma)=\int^{\oplus} \mathbb{I}_{\mathcal{L}_{\sigma}} \otimes \check{\mathbf{A}}_{\check{\sigma}}$. The fiber operators $\mathbf{A}_{\sigma}$ are ( $\nu$ a.e.) selfadjoint and therefore have a spectral resolution, which leads to a direct integral decomposition of the spaces $L_{\sigma}^{2}(\mathcal{N})$ as well as of $\mathbf{A}_{\sigma}$ and $\mathbf{A}(\sigma)$ :

$$
\begin{aligned}
L_{\sigma}^{2}(\mathcal{N})_{0} & =\int^{\oplus} d \mu_{\sigma}(\lambda) \mathcal{L}_{\lambda \sigma}^{2}(\mathcal{N}), \\
\mathbf{A}_{\sigma} & =\int^{\oplus} d \mu_{\sigma}(\lambda) \lambda \mathbb{1}_{\mathcal{L}_{\lambda \sigma}^{2}(\mathcal{N})},
\end{aligned}
$$

as well as

$$
\begin{aligned}
\mathcal{L}_{\sigma}^{2}\left(\mathcal{M}_{r}\right) & =\int^{\oplus} d \mu_{\sigma}(\lambda) \mathcal{L}_{\sigma} \otimes \mathcal{L}_{\lambda \check{\sigma}}^{2}(\mathcal{N}), \\
\mathbf{A}(\sigma) & =\int^{\oplus} d \mu_{\sigma}(\lambda) \lambda \mathbb{1}_{\mathcal{L}_{\sigma} \otimes \mathcal{L}_{\lambda \check{\sigma}}^{2}(\mathcal{N})} .
\end{aligned}
$$


Combining these decompositions, we have

$$
\begin{aligned}
L^{2}\left(\mathcal{M}_{r}\right) & =\int^{\oplus} d \nu(\sigma) d \mu_{\sigma}(\lambda) \mathcal{L}_{\sigma} \otimes \mathcal{L}_{\lambda \check{\sigma}}^{2}(\mathcal{N}), \\
\mathbf{A} & =\int^{\oplus} d \nu(\sigma) d \mu_{\sigma}(\lambda) \lambda \mathbb{I}_{\mathcal{L}_{\sigma} \otimes \mathcal{L}_{\lambda \check{\sigma}}^{2}(\mathcal{N})} .
\end{aligned}
$$

So we have identified the measure $d \mu(\lambda, \sigma)$ in (2.4) as $d \nu(\sigma) d \mu_{\sigma}(\lambda)$.

\subsection{Relating the spectral problems of $\mathrm{A}$ and $\mathrm{A}_{\sigma}: \mathbf{A}_{\sigma}$ compact}

Our goal is to analyze concretely how the spectral resolution of the fiber operators $\mathbf{A}_{\sigma}$ relates to the spectral problem of $\mathbf{A}$, including the construction of generalized eigenvectors. At first we consider an important special case in which the kernel of $\mathbf{A}$ is 'almost' square integrable implying that the fiber operators $\mathbf{A}_{\sigma}$ are compact. Explicitly we require:

(C) $\int d g d n d n^{\prime}\left|\mathcal{A}\left(g, n, n^{\prime}\right)\right|^{2}<\infty$, or equivalently $\int_{Q^{\nu-1}} d q \int d m\left|A\left(q^{\uparrow}, q, m\right)\right|^{2}<\infty$.

Here $A\left(m, m^{\prime}\right)$ is the kernel of the original operator acting on $L^{2}(\mathcal{M})$ while $\mathcal{A}$ is the kernel of its image under the isometry in Proposition 2.1. Of course since $A\left(m, m^{\prime}\right)$ is invariant it can never be square integrable in the proper sense, see Proposition 3.1. However (C) implies that the fiber operators are Hilbert-Schmidt for almost all $\sigma$.

Lemma 3.2. Let $\mathbf{A}$ be a standard invariant selfadjoint operator as in Definition 3.1 whose kernel in addition satifies condition $(C)$. Then the fiber operators $\mathbf{A}_{\sigma}$ and $\mathbf{A}(\sigma)$ are Hilbert-Schmidt for all $\sigma \in \widehat{G}_{d}$ and for almost all $\sigma \in \widehat{G}_{c}$. These $\sigma \in \widehat{G}_{r}$ are called non-exceptional.

Proof. The Parseval identity (2.36) applied to $\mathcal{A}\left(g, n, n^{\prime}\right)$ gives

$$
\int d g\left|\mathcal{A}\left(g, n, n^{\prime}\right)\right|^{2}=\int_{\widehat{G}_{r}} d \nu(\sigma) \operatorname{Tr}\left[\widehat{\mathcal{A}}\left(\sigma, n, n^{\prime}\right)^{\dagger} \widehat{\mathcal{A}}\left(\sigma, n, n^{\prime}\right)\right]
$$

The integral over $n, n^{\prime}$ of the left hand side is finite by assumption; that of the right hand side can be expressed in terms of the Hilbert-Schmidt norm of the integral operator $\mathbf{A}(\sigma)$ and gives $\int d \nu(\sigma)\|\mathbf{A}(\sigma)\|_{2}^{2}$. Thus $\mathbf{A}(\sigma)$ must have finite Hilbert-Schmidt norm for almost all $\sigma \in \widehat{G}_{r}$, as asserted. The equivalence to the second condition in (C) can be seen from (3.2). In detail

$$
\begin{aligned}
& \int d g d n d n^{\prime}\left|\mathcal{A}\left(g, n, n^{\prime}\right)\right|^{2}=\int d g d n d n^{\prime}\left|A\left(g q^{\uparrow}, g n, q_{0}, n^{\prime}\right)\right|^{2} \\
& =\int d \gamma_{Q}\left(q_{1}\right) d n d n^{\prime}\left|A\left(q_{1}, g_{s}\left(q_{1}\right) n, q^{\uparrow}, n^{\prime}\right)\right|^{2}=\int_{Q^{\nu-1}} d q \int d m\left|A\left(q^{\uparrow}, q, m\right)\right|^{2} .
\end{aligned}
$$


In the second equality we used that the fact that the section $g_{s}: Q \rightarrow G$ provides a one-to-one correspondence between points in $Q$ and right $K$ orbits in $G$. By definition $\left(q_{1}, g_{s}\left(q_{1}\right) n\right)=\left(q_{1}, q_{2}, \ldots q_{\nu}\right)=m$ and the $d \gamma_{Q}\left(q_{1}\right) d n=d \gamma(m)$ integral just defines the iteration of the modulus of the kernel.

The spectral problem for the fiber operators $\mathbf{A}_{\sigma}$ and $\mathbf{A}(\sigma)$ is now trivial: these operators have discrete spectrum except for 0 , which is an accumulation point of eigenvalues of finite multiplicity. All eigenvectors lie in the respective $L^{2}$ spaces, i.e. in $L_{\sigma}^{2}(\mathcal{N})$ for $\mathbf{A}_{\sigma}$ and in $L_{\sigma \check{\sigma}}^{2}(\mathcal{N})$ for $\mathbf{A}(\sigma)$. Since $\mathbf{A}(\sigma)$ is of the form $\mathbb{I} \otimes \check{\mathbf{A}}_{\check{\sigma}}$ a dense set of eigenvectors $F: \mathcal{N} \rightarrow \mathcal{L}_{\sigma} \otimes \check{\mathcal{L}}_{\check{\sigma}}$ exists such that $F(n)$ is trace class (not just Hilbert-Schmidt as in the definition of $L_{\sigma \check{\sigma}}^{2}(\mathcal{N})$. The goal in the following is to 'lift' these normalizable eigenvectors of the fiber operators to non-normalizable $\sigma$-equivariant eigenfunctions of $\mathbf{A}$. To this end the action of $\mathbf{A}$ has to be extended to functions outside of $L^{2}\left(\mathcal{M}_{r}\right)$. Our Definition 3.1 ensures that $\mathbf{A}$ can be extended naturally as an operator from $L^{\infty}\left(\mathcal{M}_{r}\right)$ to itself, bounded in the sup norm. In order to relate the eigenvectors of the fiber operators to the eigenfunctions of $\mathbf{A}$ (which will no longer be square integrable), we need a generalization of the Fourier transformation.

In preparation we introduce two pairs of Banach spaces as follows:

\section{Definition 3.2.}

$$
\begin{array}{ll}
L^{1,2}(G \times \mathcal{N}):=L^{1}(G) \otimes L^{2}(\mathcal{N}), & \|\phi\|^{1,2}:=\left[\int d n\left(\int d g|\phi(g, n)|\right)^{2}\right]^{1 / 2}, \\
L^{\infty, 2}(G \times \mathcal{N}):=L^{\infty}(G) \otimes L^{2}(\mathcal{N}), & \|t\|^{\infty, 2}:=\left[\int d n\left(\sup _{g}|t(g, n)|\right)^{2}\right]^{1 / 2} .
\end{array}
$$

Further, for $\sigma \in \widehat{G}_{r}$

$$
L^{2}\left(\mathcal{N}, \mathcal{C}\left(\mathcal{L}_{\sigma}\right)\right):=\left\{C: \mathcal{N} \rightarrow \mathcal{C}\left(\mathcal{L}_{\sigma}\right) \mid \int d n\|C(n)\|^{2}<\infty\right\}
$$

where $\mathcal{C}\left(\mathcal{L}_{\sigma}\right)$ is the space of compact operators on $\mathcal{L}_{\sigma}$ and $\|C(n)\|$ is the operator norm. Similarly

$$
L^{2}\left(\mathcal{N}, \mathcal{J}_{1}\left(\mathcal{L}_{\sigma}\right)\right):=\left\{F: \mathcal{N} \rightarrow \mathcal{J}_{1}\left(\mathcal{L}_{\sigma}\right) \mid \int d n\|F(n)\|_{1}^{2}<\infty\right\}
$$

where $\mathcal{J}_{1}\left(\mathcal{L}_{\sigma}\right)$ is the space of trace class operators on $\mathcal{L}_{\sigma}$ and $\|F(n)\|_{1}=\operatorname{Tr}[|F(n)|]$ is the trace norm of $F(n)$.

Denoting by ' the topological duals one has

$$
\begin{gathered}
L^{\infty, 2}(\mathcal{N} \times G)=L^{1,2}(G \times \mathcal{N})^{\prime} \\
L^{2}\left(\mathcal{N}, \mathcal{J}_{1}\left(\mathcal{L}_{\sigma}\right)\right)=L^{2}\left(\mathcal{N}, \mathcal{C}\left(\mathcal{L}_{\sigma}\right)\right)^{\prime}
\end{gathered}
$$


Of course the spaces are not reflexive, the double duals are much larger than the original spaces. The relations (3.30) follow from the well-known facts $L^{1}(G)^{\prime}=L^{\infty}(G)$ and $\mathcal{C}\left(\mathcal{L}_{\sigma}\right)^{\prime}=\mathcal{J}_{1}\left(\mathcal{L}_{\sigma}\right)$, see e.g. [43], Theorem VI.26, for the latter. Concretely one has $|(t, \phi)| \leq\|t\|^{\infty, 2}\|\phi\|^{1,2}, t \in L^{\infty, 2}, \phi \in L^{1,2}$. Thus $(t, \cdot)$ defines a linear bounded functional on $L^{1,2}$; moreover every such functional is of that form. Similarly,

$$
(F, C)_{\sigma \check{\sigma}}=\int d n \operatorname{Tr}\left[F(n)^{\dagger} C(n)\right] \leq\left[\int d n\|F(n)\|_{1}^{2}\right]^{1 / 2}\left[\int d n\|C(n)\|^{2}\right]^{1 / 2}
$$

so that $(F, \cdot)_{\sigma \check{\sigma}}$ defines a linear bounded functional on $L^{2}\left(\mathcal{N}, \mathcal{C}\left(\mathcal{L}_{\sigma}\right)\right)$; moreover every such functional is of this form.

The two pairs of spaces $(3.30, b)$ are related by the generalization of the Fourier transform we are aiming for. To see this we introduce

Definition 3.3. A function $t=t_{\sigma} \in L^{\infty, 2}(G \times \mathcal{N})$ is called $\sigma$-equivariant for some $\sigma \in \widehat{G}_{r}$, if there exists a $\ell_{\sigma} \in L^{2}\left(\mathcal{N}, \mathcal{C}\left(\mathcal{L}_{\sigma}\right)\right)^{\prime}$ such that

$$
\left(t_{\sigma}, \phi\right)=\ell_{\sigma}(\widehat{\phi}(\sigma)) \quad \text { for all } \phi \in L^{1,2}(G \times \mathcal{N})
$$

where $\widehat{\phi}(\sigma, n)$ is the Fourier transform (2.33) of $\phi$ (which is $\mathcal{C}\left(\mathcal{L}_{\sigma}\right)$-valued for $\phi(\cdot, n) \in$ $\left.L^{1}(G)\right)$. Such $t_{\sigma}$ intertwine the right $G$ action $\rho$ on $L^{\infty}(G)$ with that of $\pi_{\sigma}$, i.e.

$$
\left(\rho\left(g^{-1}\right) t_{\sigma}, \phi\right)=\ell_{\sigma}\left(\pi_{\sigma}(g) \widehat{\phi}\right)
$$

for all $g \in G$ and $\phi \in L^{1,2}(G \times \mathcal{N})$. The $\sigma$-equivariant subspace of $L^{\infty, 2}(G \times \mathcal{N})$ is denoted by $\mathcal{L}_{\sigma}^{\infty, 2}(G \times \mathcal{N})$.

Proposition 3.5. Every $\sigma$-equivariant $t_{\sigma} \in L^{\infty, 2}(G \times \mathcal{N})$ is of the form

$$
t_{\sigma}^{F}(g, n)=\operatorname{Tr}\left[F(n) \pi_{\sigma}(g)\right]
$$

for a unique $F \in L^{2}\left(\mathcal{N}, \mathcal{J}_{1}\left(\mathcal{L}_{\sigma}\right)\right)$. Equivalently the map $t_{\sigma}: L^{2}\left(\mathcal{N}, \mathcal{J}_{1}\left(\mathcal{L}_{\sigma}\right)\right) \rightarrow \mathcal{L}_{\sigma}^{\infty, 2}(G \times$ $\mathcal{N}), F \mapsto t_{\sigma}^{F}$, is a bounded injection. The intertwining relation becomes $t_{\sigma}^{\pi_{\sigma}\left(g_{0}\right) F}(g, n)=$ $t_{\sigma}^{F}\left(g g_{0}, n\right)$. A substitute for the Parseval relation is

$$
\left(t_{\sigma}^{F}, \phi\right)=(F, \widehat{\phi}(\sigma))_{\sigma \check{\sigma}}
$$

for all $\phi \in L^{1,2}(G \times \mathcal{N})$.

Proof. We begin by showing that the map $F(n) \mapsto t_{\sigma}^{F}(\cdot, n)$ is injective for almost all $n \in \mathcal{N}$. 
Here we can appeal to a theorem due to Glimm [18, 15] that implies that for a type I group (which we have here) the closure in operator norm of the algebra generated by $\pi_{\sigma}(g), g \in G$ consists of all compact operators on $\mathcal{L}_{\sigma}$. Then if $t_{\sigma}^{F}(\phi)=0$ for all $\phi \in \mathcal{D}(G)$ also $\operatorname{Tr}\left[F \pi_{\sigma}(g)\right]=0$ for all $g \in G$ and by the above mentioned theorem $\operatorname{Tr}[F A]=0$ for all compact $A$, which implies $F=0$.

A slightly more down to earth argument goes as follows: Assume again that $\operatorname{Tr} F \pi_{\sigma}(\phi)=$ 0 for all $\phi \in \mathcal{D}(G)$. Replacing $\phi$ by $E_{\kappa} * \phi * E_{\kappa^{\prime}}$ we see that $\operatorname{Tr}\left[P_{\kappa} F P_{\kappa^{\prime}} \pi_{\sigma}(\phi)\right]=0$ for all $\phi \in \mathcal{D}(G)$ and $\kappa, \kappa^{\prime}$. Note that $\operatorname{Ran} P_{\kappa}=m_{\kappa} V_{\kappa}$ and $\operatorname{Ran} P_{\kappa}^{\prime}=m_{\kappa^{\prime}} V_{\kappa^{\prime}}$ have finite dimension. By a theorem of Kadison [28, the norm closure of the algebra generated by $\pi_{\sigma}(g), g \in G$, contains operators that map any finite set of linearly independent vectors into any other given finite set of vectors of the same cardinality. This means that for any linear map $L_{\kappa^{\prime}, \kappa}$ from $m_{\kappa} V_{\kappa}$ to $m_{\kappa^{\prime}} V_{\kappa^{\prime}}$ there is an element $A$ in that norm closure such that $L_{\kappa^{\prime}, \kappa}=P_{\kappa^{\prime}} A P_{\kappa}$. Hence $\operatorname{Tr}\left[F L_{\kappa^{\prime}, \kappa}\right]=0$ for any such linear map, which implies $F=0$.

To complete the proof, let $\ell_{\sigma} \in L^{2}\left(\mathcal{N}, \mathcal{C}\left(\mathcal{L}_{\sigma}\right)\right)^{\prime}$ be the element associated with $t_{\sigma}$ via (3.32). On account of (3.30b) there exist some $F \in L^{2}\left(\mathcal{N}, \mathcal{J}_{1}\left(\mathcal{L}_{\sigma}\right)\right)$ such that $\ell_{\sigma}(C)=$ $\left(F^{\dagger}, C\right)_{\sigma \check{\sigma}}$ for all $C \in L^{2}\left(\mathcal{N}, \mathcal{C}\left(\mathcal{L}_{\sigma}\right)\right)$. This holds in particular for $C(\sigma, n)=\widehat{\phi}(\sigma, n)$ for $\phi \in L^{1}(G) \otimes L^{2}(\mathcal{N})$. A simple computation then gives $\ell_{\sigma}(\widehat{\phi}(\sigma))=\left(t_{\sigma}^{F}, \phi\right)$, with $t_{\sigma}^{F}$ as in (3.34). The uniqueness of $F$ follows the above injectivity result. For the norm of $t_{\sigma}^{F}$ one has $\left\|t_{\sigma}^{F}\right\|^{\infty, 2} \leq\left(\int d n\|F(n)\|_{1}^{2}\right)^{1 / 2}$.

We add some remarks. Proposition 3.5 allows to give the space $\mathcal{L}_{\sigma}^{\infty, 2}(G \times \mathcal{N})$ the structure of a pre-Hilbert space by defining a scalar product

$$
\left(t_{\sigma}^{F}, t_{\sigma}^{F^{\prime}}\right):=\int d n \operatorname{Tr}\left[F(n)^{\dagger} F^{\prime}(n)\right]
$$

and of course by completion this gives rise to a Hilbert space, which can be identified with $L^{2}\left(\mathcal{N}, \mathcal{J}_{2}\left(\mathcal{L}_{\sigma}\right)\right)$, where $\mathcal{J}_{2}\left(\mathcal{L}_{\sigma}\right)$ is the space of Hilbert-Schmidt operators on $\mathcal{L}_{\sigma}$.

Comparing (3.34) with (2.33) one sees that $F$ can be viewed as the unique Fourier transform of $t=t_{\sigma}$, but in a fixed $\sigma$ fiber (it is a ' $\delta$ function' in $\widehat{G}_{r}$ ). The equivariance property (3.32) characterizes the fiber. Equivalently the $\sigma$-equivariant subspace of $L^{\infty, 2}(G \times \mathcal{N})$ can be identified with $L^{2}\left(\mathcal{N}, \mathcal{J}_{1}\left(\mathcal{L}_{\sigma}\right)\right)$ by the bounded injection $t_{\sigma}$. The map $t_{\sigma}$ can also be turned into an isometry by transporting the norm to its image.

Since $L^{2}\left(\mathcal{N}, \mathcal{J}_{1}\left(\mathcal{L}_{\sigma}\right)\right)$ carries the representation $\pi_{\sigma} \times \pi_{\check{\sigma}}, \sigma \in \widehat{G}_{r}$, of $G \times G$, where $\left(\pi_{\sigma} \times\right.$ $\left.\pi_{\check{\sigma}}\right)\left(g_{0}, g_{1}\right) F(n)=\pi_{\sigma}\left(g_{0}\right) F(n) \pi_{\sigma}\left(g_{1}^{-1}\right)$, the intertwining property in Proposition 3.5 in principle generalizes to $\left(\pi_{\sigma} \times \pi_{\check{\sigma}}\right)\left(g_{0}, g_{1}\right) t_{\sigma}^{F}(g, n)=t_{\sigma}^{F}\left(g_{1}^{-1} g g_{0}, n\right)$. However since we eventually are interested in functions on $\mathcal{M}_{r}=(G \times \mathcal{N}) / d(K)$, only left actions with $g_{1}=k \in K$ are useful.

Whenever $F(n)$ satisfies the equivariance condition (2.43) the function $t_{\sigma}^{F}(g, n)$ projects to one on $\mathcal{M}_{r}$, i.e. $t_{\sigma}^{F}(k g, k n)=t_{\sigma}^{F}(g, n)$ for $k \in K$. We denote the left $K$ invariant 
subspace of $L^{\infty, 2}(G \times \mathcal{N})$ by $L^{\infty, 2}\left(\mathcal{M}_{r}\right)$ and the subspace of $L^{2}\left(\mathcal{N}, \mathcal{J}_{1}\left(\mathcal{L}_{\sigma}\right)\right)$ whose elements satisfy (2.43) by $L^{2}\left(\mathcal{N}, \mathcal{J}_{1}\left(\mathcal{L}_{\sigma}\right)\right)_{0}$. Proposition 3.5 evidently remains valid as an injective identification of $L^{2}\left(\mathcal{N}, \mathcal{J}_{1}\left(\mathcal{L}_{\sigma}\right)\right)_{0}$ with the $\sigma$-equivariant subspace of $L^{\infty, 2}\left(\mathcal{M}_{r}\right)$, for which we write $\mathcal{L}_{\sigma}^{\infty, 2}\left(\mathcal{M}_{r}\right)$.

To proceed we now show that in addition to intertwining the group actions, the map $t_{\sigma}$ also intertwines the action of $\mathbf{A}$ with that of $\mathbf{A}(\sigma)$. Indeed, using only the definitions (3.7), (3.13), (3.34) one computes

$$
\begin{aligned}
{\left[\mathbf{A} t_{\sigma}^{F}\right](g, n) } & =\int d n^{\prime} \operatorname{Tr}\left[F\left(n^{\prime}\right) \widehat{\mathcal{A}}\left(\sigma, n^{\prime}, n\right) \pi_{\sigma}(g)\right] \\
& =\operatorname{Tr}\left[(\mathbf{A}(\sigma) F)(n) \pi_{\sigma}(g)\right]=t_{\sigma}^{\mathbf{A}(\sigma) F}(g, n) .
\end{aligned}
$$

If the Fourier transform were well-defined on these functions the relation (3.37) would amount to $\widehat{\mathbf{A} t_{\sigma}^{F}}=\mathbf{A}(\sigma) F$, just as in (3.14), (3.15). The point of (3.37) is that it remains valid for $\mathbf{A}$ acting on $\mathcal{L}_{\sigma}^{\infty, 2}(G \times \mathcal{N})$ and $\mathbf{A}(\sigma)$ acting on $L_{\sigma \check{\sigma}}^{2}(\mathcal{N})$.

Since $n \mapsto F(n)$ takes values in the trace class operators and the kernel $\widehat{\mathcal{A}}$ acts from the right one can expand $F(n)$ into rank 1 operators of the form

$$
F(n)=\sum_{i=1}^{\infty} v_{i} \otimes \check{f}_{i}(n)
$$

with $v_{i} \in \mathcal{L}_{\sigma}$ and $f_{i}: \mathcal{N} \rightarrow \mathcal{L}_{\sigma}$ in $L_{\sigma}^{2}(\mathcal{N})$. Mostly it is therefore the restriction of $t_{\sigma}$ to rank 1 operators $F(n)=v \otimes \check{f}(n)$ that is needed and it is convenient to introduce a separate notation for it. We define

$$
\begin{aligned}
& \tau_{v \sigma}: L_{\sigma}^{2}(\mathcal{N}) \rightarrow \mathcal{L}_{\sigma}^{\infty, 2}(G \times \mathcal{N}), \quad f \mapsto \tau_{v \sigma}(f), \\
& \tau_{v \sigma}(f)(g, n):=t_{\sigma}^{v \otimes \check{f}}(g, n)=\operatorname{Tr}\left[(v \otimes \check{f}(n)) \pi_{\sigma}(g)\right]=\left(f(n), \pi_{\sigma}(g) v\right)_{\sigma} .
\end{aligned}
$$

Note that the map is antilinear in $f$ but linear in $\check{f}$, and that $t_{\sigma}^{F}(g, n)=\sum_{i} \tau_{v_{i} \sigma}\left(f_{i}\right)(g, n)$, for $F$ of the form (3.38). Whenever $f$ is $K$ equivariant the image function is left $K$ invariant, i.e. (3.39) also defines a map $\tau_{v \sigma}: L_{\sigma}^{2}(\mathcal{N})_{0} \rightarrow \mathcal{L}_{\sigma}^{\infty, 2}\left(\mathcal{M}_{r}\right)$. It is straightforward to see that the map is bounded in the norm $\|\cdot\|^{\infty, 2}$. It is thus a bounded injection and can be made into an isometry onto its image by transferring the norm to the image. The representation $\pi_{\sigma}$ acts on rank 1 valued operators merely by rotating the reference vector: $v \otimes \check{f}(n) \mapsto\left(\pi_{\sigma}\left(g_{0}\right) v\right) \otimes \check{f}(n)$, so that $\tau_{v \sigma}(f)\left(g g_{0}, n\right)=\tau_{\pi_{\sigma}\left(g_{0}\right) v, \sigma}(f)(g, n)$. Further $\tau_{v \sigma}$ interwines the action of $\mathbf{A}$ and $\mathbf{A}_{\sigma}$ : either from (3.37) or directly from (3.13), (3.18) one finds

$$
\left(\mathbf{A} \tau_{v \sigma}(f)\right)(g, n)=\left(\mathbf{A}_{\sigma} f_{\sigma}(n), \pi_{\sigma}(g) v\right)_{\sigma}=\left(\tau_{v \sigma}\left(\mathbf{A}_{\sigma} f\right)\right)(g, n) .
$$

This intertwining relation now allows to reduce the spectral problem for $\mathbf{A}$ to the simpler spectral problems of the fiber operators $\mathbf{A}_{\sigma}$ in the decomposition $\mathbf{A}=\int^{\oplus} d \nu(\sigma)\left(\mathbb{I} \otimes \check{\mathbf{A}}_{\check{\sigma}}\right)$. 
We write

$$
\begin{aligned}
& \mathcal{E}_{\lambda, \sigma}(\mathbf{A})=\left\{\Omega_{\sigma} \in \mathcal{L}_{\sigma}^{\infty, 2}\left(\mathcal{M}_{r}\right) \mid \Omega_{\sigma}\right. \text { is eigenfunction of } \\
& \mathbf{A} \text { with spectral value } \lambda \in \operatorname{Spec}(\mathbf{A})\} \\
& \mathcal{E}_{\lambda}\left(\mathbf{A}_{\sigma}\right)=\left\{\chi_{\sigma} \in L_{\sigma}^{2}(\mathcal{N})_{0} \mid \chi_{\sigma}\right. \text { is eigenvector of } \\
& \left.\mathbf{A}_{\sigma} \text { with eigenvalue } \lambda \in \operatorname{Spec}\left(\mathbf{A}_{\sigma}\right)\right\}
\end{aligned}
$$

and similarly for $\mathcal{E}_{\lambda}\left(\check{\mathbf{A}}_{\check{\sigma}}\right) \subset L_{\check{\sigma}}^{2}(\mathcal{N})$ and $\mathcal{E}_{\lambda}(\mathbf{A}(\sigma)) \subset L^{2}\left(\mathcal{N}, \mathcal{J}_{1}\left(\mathcal{L}_{\sigma}\right)\right)_{0}$.

The space $\mathcal{E}_{\lambda, \sigma}(\mathbf{A})$ inherits the pre-Hilbert space structure from $\mathcal{L}_{\sigma}^{\infty, 2}\left(\mathcal{M}_{r}\right)$ and we denote its completion by $\overline{\mathcal{E}_{\lambda, \sigma}(\mathbf{A})}$, whereas $\mathcal{E}_{\lambda}\left(\mathbf{A}_{\sigma}\right)$ is already a Hilbert space.

The relation of these spectral problems is described by

Proposition 3.6. Let $\mathbf{A}$ be a standard invariant selfadjoint operator on $L^{2}\left(\mathcal{M}_{r}\right)$ in the sense of Definition 3.1 and assume that its kernel satisfies in addition condition $(C)$. Then the fiber operators $\mathbf{A}(\sigma), \mathbf{A}_{\sigma}$ are Hilbert-Schmidt for all non-exceptional $\sigma \in \widehat{G}_{r}$ by Lemma 3.2. Further for these $\sigma \in \widehat{G}_{r}$

(a) For each $v \in \mathcal{L}_{\sigma}$ the map $\tau_{v \sigma}: \mathcal{E}_{\lambda}\left(\mathbf{A}_{\sigma}\right) \rightarrow \mathcal{E}_{\lambda, \sigma}(\mathbf{A})$ is a bounded injection.

(b) If $\mathbf{A}_{\sigma}, \sigma \in \widehat{G}_{d}$, has an eigenvector (normalizable eigenfunction) for some $\lambda$, the associated generalized eigenfunction in (a) of $\mathbf{A}$ is normalizable (i.e. $\lambda$ is an eigenvalue of $\mathbf{A}$ of infinite multiplicity).

(c) A complete orthonormal set of eigenfunctions of $\mathbf{A}(\sigma)$ in $\mathcal{E}_{\lambda}(\mathbf{A}(\sigma))$ is given by

$$
\left\{e_{i} \otimes \check{\chi}_{\lambda \sigma r}\right\}
$$

a complete orthonormal set of eigenfunctions $\mathbf{A}$ in $\overline{\mathcal{E}_{\lambda, \sigma}(\mathbf{A})}$ is given by

$$
\left\{\tau_{e_{i} \sigma}\left(\chi_{\lambda \sigma r}\right)(g, n)\right\}
$$

where $\chi_{\lambda \sigma r}$ runs through an orthonormal basis of $\mathcal{E}_{\lambda}\left(\mathbf{A}_{\sigma}\right)$ and $e_{i}$ runs through an orthonormal basis of $\mathcal{L}_{\sigma}$. The eigenfunctions (3.43) vanish pointwise for $g \rightarrow \infty$ (i.e. as $g$ leaves any compact subset of $G$ ).

$\overline{\mathcal{E}_{\lambda, \sigma}(\mathbf{A})}$ and $\mathcal{E}_{\lambda}(\mathbf{A}(\sigma))$ are isometric as Hilbert spaces. In particular the spectrum of $\mathbf{A}(\sigma)$ is contained in the spectrum of $\mathbf{A}$ for almost all $\sigma \in \widehat{G}_{r}$. 
(d) An orthonormal basis of $L_{\sigma}^{2}(\mathcal{N})_{0}$ is given by

$$
\left\{\chi_{\lambda \sigma r}\right\}
$$

where $\chi_{\lambda \sigma r}$ runs through an orthonormal basis of $\mathcal{E}_{\lambda}\left(\mathbf{A}_{\sigma}\right)$, and $\lambda$ runs through the eigenvalues of $\mathbf{A}_{\sigma}$. An orthonormal basis of $L^{2}\left(\mathcal{N}, \mathcal{J}_{2}\left(\mathcal{L}_{\sigma}\right)\right)_{0}$ is given by

$$
\left\{e_{i} \otimes \check{\chi}_{\lambda \sigma r}\right\}
$$

where in addition $e_{i}$ runs through an orthonormal basis of $\mathcal{L}_{\sigma}$.

(e) The set of all eigenfunctions $\bigcup_{\lambda \sigma} \mathcal{E}_{\lambda, \sigma}(\mathbf{A})$ is complete in the sense that for each $\phi \in\left(L^{2} \cap L^{1}\right)\left(\mathcal{M}_{r}\right)$ the following Parseval relation holds:

$$
(\phi, \phi)=\int d \nu(\sigma) d \mu_{\sigma}(\lambda) \sum_{i, r} \int d n\left|\left(\chi_{\lambda \sigma r}(n), \widehat{\phi}(\sigma, n) e_{i}\right)_{\sigma}\right|^{2}
$$

where the measure $d \mu_{\sigma}(\lambda)$ introduced in (3.23) here is just the counting measure of the eigenvalues $\lambda$ of $\mathbf{A}_{\sigma}$.

Proof. (a) Since $\mathbf{A}_{\sigma}$ is selfadjoint, we can assume that $\lambda$ is real. Clearly, if $\chi \in \mathcal{E}_{\lambda}\left(\mathbf{A}_{\sigma}\right)$, i.e. $\mathbf{A}_{\sigma} \chi=\lambda \chi$, then $\mathbf{A} \tau_{v \sigma}(\chi)=\tau_{v \sigma}\left(\mathbf{A}_{\sigma} \chi\right)=\lambda \tau_{v \sigma}(\chi)$, i.e. $\tau_{v \sigma}(\chi) \in \mathcal{E}_{\lambda, \sigma}(\mathbf{A})$. By Proposition 3.5 the map $\mathcal{E}_{\lambda}\left(\mathbf{A}_{\sigma}\right) \ni \chi \mapsto \tau_{v \sigma}(\chi) \in \mathcal{E}_{\lambda, \sigma}(\mathbf{A})$ is injective.

(b) Recall from 9], Theorem 14.3.3, that the coefficients of a square integrable representation $\pi_{\sigma}, \sigma \in \widehat{G}_{d}$, satisfy, using the $K$-adapted basis (2.46)

$$
\int d g \pi_{\sigma}(g)_{\kappa_{1} s_{1}, \kappa_{2} s_{2}}^{*} \pi_{\sigma}(g)_{\kappa_{3} s_{3}, \kappa_{4} s_{4}}=d_{\sigma}^{-1} \delta_{\kappa_{1} \kappa_{3}} \delta_{s_{1} s_{3}} \delta_{\kappa_{2} \kappa_{4}} \delta_{s_{2} s_{4}}
$$

where $d_{\sigma}$ is the formal degree of $\pi_{\sigma}$. Let $\chi \in L_{\sigma}^{2}(\mathcal{N})$ be the normalizable eigenfunction of $\mathbf{A}_{\sigma}$ for some $\sigma \in \widehat{G}_{d}$, and consider the associated generalized eigenfunction $\tau_{v \sigma}(\chi)$ of A. Using (3.47) one can verify by direct computation that

$$
d_{\sigma} \int d g d n\left|\tau_{v \sigma}(\chi)(g, n)\right|^{2}=(v, v)_{\sigma}(\chi, \chi)_{\sigma}
$$

holds. It follows that $\tau_{v \sigma}(\chi)$ is actually normalizable, i.e. an element of $L^{2}\left(\mathcal{M}_{r}\right) \cap$ $\mathcal{L}_{\sigma}^{\infty, 2}\left(\mathcal{M}_{r}\right)$. For the spectral values this implies: if $\lambda \in \mathrm{d}-\operatorname{Spec}\left(\mathbf{A}_{\sigma}\right)$ then it is an eigenvalue of infinite multiplicity of $\mathbf{A}$. 
(c) (3.42) follows directly from the completeness of the eigenfunctions of $\mathbf{A}_{\sigma}$ and the relation between the operators $\mathbf{A}(\sigma)$ and $\mathbf{A}_{\sigma}$. The second statement follows from the fact that all the functions in $\overline{\mathcal{E}_{\lambda, \sigma}(\mathbf{A})}$ are $\sigma$-equivariant and hence by Proposition 3.5 of the form $\left.\Omega_{\sigma}(g, n)=\operatorname{Tr}\left[F(n) \pi_{\sigma}(g)\right)\right]$. The eigenfunction condition translates into the condition that $F$ is an eigenvector of $\mathbf{A}(\sigma)$ with eigenvalue $\lambda$; a complete set of such eigenvectors is given by (3.42), as we have just seen. By construction the map $F \mapsto t_{\sigma}^{F}$ preserves the scalar product, which implies the orthonormality of the set (3.43). So $t_{\sigma}^{F}$ provides an isometry between $\overline{\mathcal{E}_{\lambda, \sigma}(\mathbf{A})}$ and $\mathcal{E}_{\lambda}(\mathbf{A}(\sigma))$. The vanishing at infinity in $G$ of the eigenfunctions (3.42) follows from the Howe-Moore theorem, see 60], Theorem 2.2.20.

(d) This follows from the spectral theorem applied to $\mathbf{A}_{\sigma}$ and $\mathbf{A}(\sigma)$ together with part (c).

(e) Since $\phi \in L^{2} \cap L^{1}$, its partial Fourier transform $\widehat{\phi}(\sigma, n)$ is a.e. a Hilbert-Schmidt operator in $\mathcal{L}_{\sigma}$. The statement then follows from the completeness of the fiber eigenvectors (part (a)) and the Plancherel theorem of Appendix A1.

We repeat a cautioning remark made in Section 2.2: the fact that $\lambda_{0} \in \operatorname{Spec}\left(\mathbf{A}_{\sigma_{0}}\right)$ for some $\sigma_{0}$ and hence $\mathcal{E}_{\lambda_{0}, \sigma_{0}}(\mathbf{A})$ is nontrivial does in itself not imply that $\lambda_{0} \in \operatorname{Spec}(\mathbf{A})$. In fact there are explicit counterexamples. By Proposition $3.4 \mathrm{~b}$ one needs a set $\Sigma$ of nonzero Plancherel measure, such that for each neighborhood $U$ of $\lambda_{0}$ we have $\bigcup_{\sigma \in \Sigma} \operatorname{Spec}\left(\mathbf{A}_{\sigma}\right) \cap$ $U \neq \emptyset$.

In the case of compact fiber operators $\mathbf{A}_{\sigma}$ we have however obtained the following relation between the spectral problems of $\mathbf{A}$ and $\mathbf{A}_{\sigma}$ : for every spectral value $|\lambda| \leq\|\mathbf{A}\|$ of $\mathbf{A}$ there is an eigenvalue of $\mathbf{A}_{\sigma}$. The corresponding normalizable eigenvector $\chi$ of $\mathbf{A}_{\sigma}$ generates a $G$ orbit of $\mathcal{L}_{\sigma}^{\infty, 2}$ eigenfunctions of $\mathbf{A}$ via $\tau_{v \sigma}(\chi)$, and all $\sigma$-equivariant eigenfunctions of $\mathbf{A}$ arise in that way by taking linear combinations in $\chi$ and the reference vectors $v \in \mathcal{L}_{\sigma}$. In addition there may be 'fake' solutions of $\mathbf{A} \Omega=\lambda \Omega$, where $\lambda \neq$ $\operatorname{Spec}(\mathbf{A})$. The value $|\lambda|=\|A\|$ of course always belongs to the spectrum of $\mathbf{A}$.

\subsection{Relating the spectral problems of $\mathrm{A}$ and $\mathrm{A}_{\sigma}: \mathrm{A}_{\sigma}$ not necessarily compact}

The discussion becomes a little more complicated in the case of not necessarily compact fiber operators. These naturally arise if the original kernel is not 'almost' square integrable as in condition $(\mathrm{C})$. In this section we require instead the weaker conditions

(C1) $\sup _{m} \int d m^{\prime}\left|A\left(m, m^{\prime}\right)\right|^{2}<\infty$,

(C2) There is an invariant multiplication operator $M$ given by a $d(G)$ invariant function in $L^{2}(\mathcal{M})$ also denoted by $M$, such that $M^{-1} \mathbf{A} M$ defines a bounded (invariant) operator on $L^{2}(\mathcal{M})$. 
For a positive kernel $A\left(m, m^{\prime}\right)>0$ the condition (C1) amounts to $\sup _{m} A(m, m ; 2)<$ $\infty$, which is weaker than the integrability condition in Lemma 3.1 (at least as far as decay properties of $A$ are concerned). The multiplication operator acts by $(M \psi)(m)=$ $M(m) \psi(m)$, for $\psi \in L^{2}(\mathcal{M})$. Invariance $M(d(g) m)=M(m)$, for all $g \in G$, means that $M$ maps into multiplication by a function $\tilde{M}$ on $\mathcal{N}$, given by

$$
\tilde{M}(n):=M\left(q^{\uparrow}, n\right)
$$

under the isometry $\Phi^{-1}: L^{2}(\mathcal{M}) \rightarrow L^{2}\left(\mathcal{M}_{r}\right)$ in (2.32). We drop the tilde and continue to write $M \in L^{2}(\mathcal{N})$ for this function and note that the kernel of $M^{-1} \mathbf{A} M$, viewed as a bounded operator on $L^{2}\left(\mathcal{M}_{r}\right)$ is given by $M(n)^{-1} \mathcal{A}\left(g g^{\prime-1}, n, n^{\prime}\right) M\left(n^{\prime}\right)$. The counterpart of Lemma 3.2 is now given by

Lemma 3.3. Under the conditions (C1), (C2) the fiber operators $\mathbf{A}_{\sigma}$ have for all $\sigma \in \widehat{G}_{d}$ and for allmost all $\sigma \in \widehat{G}_{c}$ the properties

(C1') $\sup _{n} \int d n^{\prime}\left\|\widehat{\mathcal{A}}\left(\sigma, n, n^{\prime}\right)\right\|_{2}^{2}=: C_{A}<\infty$, where $\|\cdot\|_{2}$ denotes the Hilbert-Schmidt norm for operators on $\mathcal{L}_{\sigma}$.

(C2') there is a function $M \in L^{2}(\mathcal{N})$ such that $M^{-1} \mathbf{A}_{\sigma} M$ defines a bounded operator on $L_{\sigma}^{2}(\mathcal{N})$, where $M$ maps each $L_{\sigma}^{2}(\mathcal{N})$ into itself via $(M \phi)(n):=M(n) \phi(n)$.

The goal in the following will be to first gain control over the eigenfunctions $\mathbf{A}_{\sigma}$ (a step which was trivial in Section 3.3) and then to lift them to $\pi_{\sigma}$ equivariant generalized eigenfunctions of $\mathbf{A}$.

Proof. (C1') follows from (C1) by applying (3.26).$\left(\mathrm{C} 2{ }^{\prime}\right)$ is obvious.

For the first step we will make use of the Gel'fand-Maurin theory of generalized eigenvectors and eigenspaces, in particular of a version due to Berezanskii as described (and proven) in Maurin [34]. Berezanskii's construction, applied to the present situation, requires to set up for almost each $\sigma \in \widehat{G}_{r}$ a triplet

$$
\Phi_{\sigma}(\mathcal{N}) \subset L_{\sigma}^{2}(\mathcal{N}) \subset \Phi_{\sigma}^{\prime}(\mathcal{N})
$$

in which $\Phi_{\sigma}=\Phi_{\sigma}(\mathcal{N})$ is the domain of an unbounded, densely defined closed operator $B_{\sigma}$ whose inverse is Hilbert-Schmidt and $\Phi_{\sigma}^{\prime}=\Phi_{\sigma}^{\prime}(\mathcal{N})$ is the topological dual of $\Phi_{\sigma}$. Choosing

$$
B_{\sigma}:=M^{-1} \mathbf{A}_{\sigma}^{-1}
$$

we compute

$$
\operatorname{Tr}_{L_{\sigma}^{2}(\mathcal{N})}\left[\left(B_{\sigma}^{-1}\right)^{\dagger}\left(B_{\sigma}^{-1}\right] \leq \int d n d n^{\prime}\left|M(n) M\left(n^{\prime}\right)\right|\left\|\widehat{\mathcal{A}}\left(\sigma, n, n^{\prime}\right)\right\|_{2}^{2} \leq\|M\|_{2}^{2} C_{A}\right.
$$


where in the last step we used property $(\mathrm{C} 1)^{\prime}$ and the fact that therefore $\left\|\widehat{\mathcal{A}}\left(\sigma, n, n^{\prime}\right)\right\|_{2}^{2}$ is the kernel of a bounded operator on $L_{\sigma}^{2}(\mathcal{N})$ with norm $\leq C_{A}$, see [30], p.176. This shows that $B_{\sigma}^{-1}$ is indeed Hilbert-Schmidt. Property (C2)' now guarantees that $\mathbf{A}_{\sigma}$ maps $\Phi_{\sigma}$ into itself. Indeed from $\mathbf{A}_{\sigma} M L_{\sigma}^{2}(\mathcal{N})=M L_{\sigma}^{2}(\mathcal{N})$ one sees that $M L_{\sigma}^{2}(\mathcal{N})$ coincides both with the range of $B_{\sigma}^{-1}=\mathbf{A}_{\sigma} M$ and with the domain of $B_{\sigma}$.

$\Phi_{\sigma}$ is a pre-Hilbert space with the scalar product

$$
(\phi, \psi)_{B}:=\left(B_{\sigma} \phi, B_{\sigma} \psi\right)_{\sigma}+(\phi, \psi)_{\sigma}
$$

we denote its completion with respect to the norm $\left\|\phi_{\sigma}\right\|_{B}:=\sqrt{(\phi, \phi)_{B}}$ by $\bar{\Phi}_{\sigma} \subset L_{\sigma}^{2}(\mathcal{N})$. The dual of $\mathbf{A}_{\sigma}$ acting on $\Phi_{\sigma}^{\prime}$ will also be denoted by $\mathbf{A}_{\sigma}$. Berezanskii's theorem guarantees that $\mathbf{A}_{\sigma}$ has a complete set of eigendistributions in $\Phi_{\sigma}^{\prime}$. We now proceed to show that in the situation at hand these are in fact almost everywhere defined functions from $\mathcal{N}$ with values in $\mathcal{L}_{\sigma}$ (which are of course not square integrable). More precisely we have

Proposition 3.7. Under the conditions (C1) and (C2) above, for almost all $\sigma \in \widehat{G}_{r}$ the fiber operator $\mathbf{A}_{\sigma}$ has a complete set of generalized eigenfunctions $\chi_{\lambda \sigma r}$, where $r=$ $1, \ldots g(\lambda)$ with $g(\lambda) \in \mathbb{N} \cup\{\infty\}$ denotes the multiplicity of the spectral value $\lambda$. The generalized eigenfunctions satisfy for almost all $\lambda \in \operatorname{Spec}\left(\mathbf{A}_{\sigma}\right)$

(a) $\mathbf{A}_{\sigma} \chi_{\lambda \sigma}=\lambda \chi_{\lambda \sigma}$

(b) $M \chi_{\lambda \sigma} \in L_{\sigma}^{2}(\mathcal{N})$.

(c) For $\phi \in M L_{\sigma}^{2}(\mathcal{N})_{0}$ we have the Parseval (completeness) relation

$$
\int d n(\phi(n), \phi(n))_{\sigma}=\int d n \int d \mu_{\sigma}(\lambda) \sum_{r=1}^{g(\lambda)}\left|\left(\chi_{\lambda \sigma r}(n), \phi(n)\right)_{\sigma}\right|^{2}
$$

We denote the space of these eigenfunctions by $\mathcal{E}_{\lambda}\left(\mathbf{A}_{\sigma}\right)$.

Proof. We first show that (the dual of) $\mathbf{A}_{\sigma}$ maps $\Phi_{\sigma}^{\prime}$ into almost everywhere defined functions from $\mathcal{N}$ with values in $\mathcal{L}_{\sigma}$. More precisely, for any $\chi \in \Phi_{\sigma}^{\prime}$

$$
M^{\dagger} \mathbf{A}_{\sigma} \chi=\left(B_{\sigma}^{-1}\right)^{\dagger} \chi \in L_{\sigma}^{2}(\mathcal{N})
$$

Since $\Phi_{\sigma}$ is a pre-Hilbert space, by the Riesz representation theorem each element $\chi$ of $\Phi_{\sigma}^{\prime}$ can be represented by an element in $\bar{\Phi}_{\sigma}$. This means that there is a $\psi_{\chi} \in \bar{\Phi}_{\sigma} \subset L_{\sigma}^{2}(\mathcal{N})$ such that for all $\phi \in \Phi_{\sigma}$

$$
\left|(\chi, \phi)_{\sigma}\right|=\left|\left(\psi_{\chi}, \phi\right)_{B}\right| \leq\left\|\psi_{\chi}\right\|_{B}\|\phi\|_{B}
$$


Replacing $\phi$ by $B_{\sigma}^{-1} \phi$, and using $\left\|B_{\sigma}^{-1} \phi\right\|_{B}^{2}=(\phi, \phi)_{\sigma}+\left(B_{\sigma}^{-1} \phi, B_{\sigma}^{-1} \phi\right)_{\sigma} \leq\left(1+\left\|B_{\sigma}^{-1}\right\|^{2}\right)\|\phi\|_{\sigma}^{2}$, gives

$$
\left|\left(\chi, B_{\sigma}^{-1} \phi\right)_{\sigma}\right| \leq\left\|\psi_{\chi}\right\|_{B}\left\|B_{\sigma}^{-1} \phi\right\|_{B} \leq \mathrm{const}\left\|\psi_{\chi}\right\|_{B}\|\phi\|_{\sigma} .
$$

This shows that $\left(\chi, B_{\sigma}^{-1} \phi\right)_{\sigma}$ defines a bounded linear functional on the dense domain $\Phi_{\sigma} \subset L_{\sigma}^{2}(\mathcal{N})$ and hence is given by an element of $L_{\sigma}^{2}(\mathcal{N})$, as claimed in (3.55). Now (a) and (c) follow from Berezanskii's theorem and (b) follows from (a) and (3.55).

Consistency requires that the dual $\mathbf{A}_{\sigma}^{\prime}$ of $\mathbf{A}_{\sigma}$ (with a separate notation only at this point) is such that $M \mathbf{A}_{\sigma}^{\prime} M^{-1}$ is a bounded operator on $L_{\sigma}^{2}(\mathcal{N})$, which is however just the relation dual to $(\mathrm{C} 2)^{\prime}$.

Let us add that it is not possible to apply this method directly to obtain the $\sigma$-equivariant generalized eigenfunctions of $\mathbf{A}$ itself. As long as $\mathbf{A}$ and $M$ are invariant the product $\mathbf{A} M$ will never be Hilbert-Schmidt, as the infinite group volume is once more overcounted. One would have to replace the invariant multiplication operator $M$ by an operator of multiplication by a (necessarily noninvariant) function in $L^{2}\left(\mathcal{M}_{r}\right)$, which would make the strategy at least cumbersome.

We rather proceed as in Section 3.3. In order to avoid having to introduce further notation we continue to use the spaces in Definition 3.2 but indicate the modified notion of square integrability by a mnemonic prefactor $M$ or $M^{-1}$. For example

$$
\begin{aligned}
& F \in M^{-1} L^{2}\left(\mathcal{N}, \mathcal{J}_{1}\left(\mathcal{L}_{\sigma}\right)\right) \quad \text { if } \quad \int d n|M(n)|^{2}\|F(n)\|_{1}^{2}<\infty, \\
& t \in M^{-1} L^{\infty, 2}(G \times \mathcal{N}) \quad \text { if } \quad \int d n|M(n)|^{2} \sup _{g}|t(g, n)|^{2}<\infty .
\end{aligned}
$$

The $\sigma$-equivariant subspace of $M^{-1} L^{\infty, 2}(G \times \mathcal{N})$ is denoted by $M^{-1} \mathcal{L}_{\sigma}^{\infty, 2}(G \times \mathcal{N})$, etc. We define $t_{\sigma}^{F}(g, n):=\operatorname{Tr}\left[F(n) \pi_{\sigma}(g)\right]$ as in (3.34). With these modified spaces Proposition 3.5 carries over, and states that every element of $M^{-1} \mathcal{L}_{\sigma}^{\infty, 2}(G \times \mathcal{N})$, is of the form $t_{\sigma}^{F}(g, n)$ with a unique $F \in M^{-1} L^{2}\left(\mathcal{N}, \mathcal{J}_{1}\left(\mathcal{L}_{\sigma}\right)\right)$. The intertwining property for the $G$ actions is manifest and $\mathbf{A} t_{\sigma}^{F}=t_{\sigma}^{\mathbf{A}(\sigma) F}$ carries over from (3.37). Since $F(n)$ is trace class we can expand it as in (3.38)

$$
F(n)=\sum_{i} v_{i} \otimes \check{\chi}_{i}(n), \quad v_{i} \in \mathcal{L}_{\sigma}, \quad \chi_{i} \in M^{-1} L_{\sigma}^{2}(\mathcal{N})
$$

and define

$$
\begin{array}{ll}
\tau_{v \sigma}: M^{-1} L_{\sigma}^{2}(\mathcal{N}) & \longrightarrow M^{-1} \mathcal{L}_{\sigma}^{\infty, 2}\left(\mathcal{M}_{r}\right), \\
\chi \mapsto \tau_{v \sigma}(\chi), & \tau_{v \sigma}(\chi)(g, n)=\left(\chi(n), \pi_{\sigma}(g) v\right)_{\sigma},
\end{array}
$$

where again the spaces and their norms are defined in the obvious way. 
In parallel to Eq. (3.41) we write

$$
\begin{aligned}
& \mathcal{E}_{\lambda, \sigma}(\mathbf{A})=\left\{\Omega_{\sigma} \in M^{-1} \mathcal{L}_{\sigma}^{\infty, 2}\left(\mathcal{M}_{r}\right) \mid \Omega_{\sigma}\right. \text { is eigenfunction of } \\
& \mathbf{A} \text { with spectral value } \lambda \in \operatorname{Spec}(\mathbf{A})\}, \\
& \mathcal{E}_{\lambda}\left(\mathbf{A}_{\sigma}\right)=\left\{\chi_{\sigma} \in M^{-1} L_{\sigma}^{2}(\mathcal{N})_{0} \mid \chi_{\sigma}\right. \text { is eigenfunction of } \\
& \left.\mathbf{A}_{\sigma} \text { with spectral value } \lambda \in \operatorname{Spec}\left(\mathbf{A}_{\sigma}\right)\right\},
\end{aligned}
$$

and similarly for $\mathcal{E}_{\lambda}\left(\check{\mathbf{A}}_{\check{\sigma}}\right) \subset M^{-1} L_{\check{\sigma}}^{2}(\mathcal{N})_{0}$ and $\mathcal{E}_{\lambda}(\mathbf{A}(\sigma)) \subset M^{-1} L^{2}\left(\mathcal{N}, \mathcal{J}_{1}\left(\mathcal{L}_{\sigma}\right)\right)_{0}$.

We obtain the following counterpart of Proposition 3.6

Proposition 3.8. Let $\mathbf{A}$ and $\mathbf{A}(\sigma), \mathbf{A}_{\sigma}$ with $\sigma \in \widehat{G}_{r}$ non-exceptional as in Proposition 3.4, and assume that $\mathbf{A}$ satisfies conditions (C1), (C2). Then

(a) $\tau_{v \sigma}: \mathcal{E}_{\lambda}\left(\mathbf{A}_{\sigma}\right) \rightarrow \mathcal{E}_{\lambda, \sigma}(\mathbf{A})$ is a bounded injection.

(b) A complete set of generalized eigenfunctions of $\mathbf{A}(\sigma)$ in $\mathcal{E}_{\lambda}(\mathbf{A}(\sigma)$ is given by

$$
\left\{e_{i} \otimes \check{\chi}_{\lambda \sigma r}\right\}
$$

a complete set of generalized eigenfunctions of $\mathbf{A}$ in $\mathcal{E}_{\lambda, \sigma}(\mathbf{A})$ is given by

$$
\left\{\tau_{e_{i} \sigma}\left(\chi_{\lambda \sigma r}\right)(g, n)\right\}
$$

where $\chi_{\lambda \sigma r}$ runs through the complete set of Propostion 3.7 and $e_{i}$ runs through an orthonormal basis of $\mathcal{L}_{\sigma}$. The eigenfunctions (3.63) vanish at infinity in $G$. $\mathcal{E}_{\lambda, \sigma}(\mathbf{A})$ and $\mathcal{E}_{\lambda}(\mathbf{A}(\sigma))$ are homeomorphic as Banach spaces, provided one uses on the image the norm transported from the preimage. In particular the spectrum of $\mathbf{A}(\sigma)$ is contained in the spectrum of $\mathbf{A}$ for almost all $\sigma \in \widehat{G}_{r}$.

(c) A complete set of of generalized eigenfunction spanning $L_{\sigma}^{2}(\mathcal{N})_{0}$ is given by

$$
\left\{\chi_{\lambda \sigma r}\right\}
$$

where $\chi_{\lambda \sigma r}$ runs through the complete set above and $\lambda$ runs through the eigenvalues of $\mathbf{A}_{\sigma}$. A complete set of eigenfunctions spanning $L^{2}\left(\mathcal{N}, \mathcal{J}_{2}\left(\mathcal{L}_{\sigma}\right)\right)_{0}$ is given by

$$
\left\{e_{i} \otimes \check{\chi}_{\lambda \sigma r}\right\}
$$

where in addition $e_{i}$ runs through an orthonormal basis of $\mathcal{L}_{\sigma}$. 
(d) The set of all eigenfunctions $\bigcup_{\lambda \sigma} \mathcal{E}_{\lambda, \sigma}(\mathbf{A})$ is complete in the sense that for each $\phi \in\left(L^{2} \cap L^{1}\right)\left(\mathcal{M}_{r}\right)$ the following Parseval relation holds:

$$
(\phi, \phi)=\int d \nu(\sigma) d \mu_{\sigma}(\lambda) \sum_{i, r} \int d n\left|\left(\chi_{\lambda \sigma r}(n), \widehat{\phi}(\sigma, n) e_{i}\right)_{\sigma}\right|^{2}
$$

with the measure $d \mu_{\sigma}(\lambda)$ introduced in (3.23).

Proof. The proof parallels that of Proposition [3.6. The only change is that the completeness of the set of eigenvectors has to be replaced by the completeness relation of Proposition 3.7 (c).

Symbolically we can summarize the content of Propositions $3.6 \mathrm{~b}$ and $3.8 \mathrm{~b}$ in the isometry

$$
\mathcal{E}_{\lambda, \sigma}(\mathbf{A}) \simeq \mathcal{E}_{\lambda}(\mathbf{A}(\sigma))
$$

That is, under the conditions $(\mathrm{C})$ or $(\mathrm{C} 1),(\mathrm{C} 2)$ the space of eigenfunctions of the fiber operator $\mathbf{A}(\sigma)$ is naturally isometric to the space of $\sigma$-equivariant eigenfunctions of $\mathbf{A}$. When $\mathbf{A}=\mathbf{T}$ is a transfer operator and the spectral value is $\lambda=\|\mathbf{T}\|$, the generalized eigenspace $\mathcal{E}_{\|\mathbf{T}\|, \sigma}(\mathbf{T})=\mathcal{G}_{\sigma}(\mathbf{T})$ is the $\sigma$-equivariant piece of ground state sector of $\mathbf{T}$.

\subsection{Absence of the discrete series}

A priori the spectral value of $\lambda$ of the operator $\mathbf{A}$ is of course unrelated to the type $\sigma$ of (irreducible) representation with respect to which a generalized eigenfunction transforms. The goal of the Section 4 will be to show that for a transfer operator $\mathbf{T}$ and the spectral value $\lambda=\|\mathbf{T}\|$, the possible representations $\sigma \in \widehat{G}_{r}$ are severely constrained. A first flavor of the correlation between the spectral value of $\mathbf{A}$ and the type $\sigma$ of irreducible representations allowed can be obtained from the

Corollary 3.1. Let $\mathbf{A}$ be as in Section 3.3 or 3.4 and $\mathbf{A}(\sigma)=\mathbb{1} \otimes \check{\mathbf{A}}_{\check{\sigma}}$ one of its fiber operators. Under any one of the following conditions the eigenspaces $\mathcal{E}_{\lambda, \sigma}(\mathbf{A}) \simeq \mathcal{E}_{\lambda}(\mathbf{A}(\sigma))$

for $\sigma \in \widehat{G}_{d}$, the discrete series of $G$, are empty:

(a) $\mathcal{E}_{\lambda}(\mathbf{A}(\sigma))$ contains a left $K$ singlet, i.e. a generalized eigenfunction $F$ obeying $F(k n)=F(n)$, for all $k \in K$.

(b) $\mathcal{E}_{\lambda, \sigma}(\mathbf{A})$ contains a right $K$ singlet, i.e. a generalized eigenfunction $\Omega$ obeying $\Omega(g k, n)=\Omega(g, n)$, for all $k \in K$.

(c) $\mathbf{A}=\mathbf{T}$ is a transfer operator, $\lambda=\|\mathbf{T}\|$ and it is an eigenvalue of $\mathbf{T}(\sigma)$. 
Proof. (a),(b) For definiteness let us consider the setting in Section 3.3, for the one in Section 3.4 only notational changes are required. Via the isometry $F \rightarrow t_{\sigma}^{F}$ the assumptions in (a) and (b) amount to $\operatorname{Tr}\left[F(k n) \pi_{\sigma}(g)\right]=\operatorname{Tr}\left[F(n) \pi_{\sigma}(g)\right]$ and $\operatorname{Tr}\left[F(n) \pi_{\sigma}(g k)\right]=$ $\operatorname{Tr}\left[F(n) \pi_{\sigma}(g)\right]$, respectively, for all $k \in K, g \in G$ and almost all $n \in \mathcal{N}$. In terms of $P_{0}$, the projector onto the $\kappa=0$ singlet subspace in $\mathcal{L}_{\sigma}$, this becomes $\operatorname{Tr}\left[F(n) \pi_{\sigma}(g)\right]=$ $\operatorname{Tr}\left[F(n) P_{0} \pi_{\sigma}(g)\right]$ and $\operatorname{Tr}\left[F(n) \pi_{\sigma}(g)\right]=\operatorname{Tr}\left[F(n) \pi_{\sigma}(g) P_{0}\right]$, respectively. However these can be nonvanishing functions only if the projections $P_{0} \pi_{\sigma}(g)$ or $\pi_{\sigma}(g) P_{0}$ are nontrivial, which requires $\pi_{\sigma}$ to be of $K$-type 1 . All $K$-type 1 representations are however non-discrete (in fact spherical principal series) representations.

(c) The proof is by contradiction. Let $\sigma \in \widehat{G}_{d}$ be given and suppose that the point spectrum of $\mathbf{T}_{\sigma}$ is nonempty and contains $\|\mathbf{T}\|$. The spectral radius equals $\|\mathbf{T}\|$ and the normalizable eigenfunction $\chi_{\sigma}$ of $\mathbf{T}_{\sigma}$ has eigenvalue $\|\mathbf{T}\|$. By Prop 3.6(b) the associated eigenfunction $\tau_{v \sigma}\left(\chi_{\sigma}\right)$ of $\mathbf{T}$ is normalizable. This contradicts Prop. 3.1(b).

Part (c) of the corollary is useful because in many cases all the fiber operators $\mathbf{T}_{\sigma}$ are compact (see Section 3.3). Then the nonzero spectrum of $\mathbf{T}_{\sigma}$ is discrete for all nonexceptional $\sigma$. Parts (a), (b) are useful, because often one has independent reasons to expect that a ground state should exist, which is at least a singlet under a maximal compact subgroup $K$ of $G$. In all cases the corollary entails that all discrete series representations are ruled out as candidates for the representation carried by the ground state sector. The remaining part of the restricted dual corresponds to noncompact Cartan subalgebras (see Appendix A.2) and the associated irreducible representations are cuspidial principal series. To avoid having to discuss the restricted dual $\widehat{G}_{r}$ of generic linear reductive Lie groups, we focus from now on on the case $G=\mathrm{SO}_{0}(1, N)$. Its representation theory shows all the typical complications (existence of discrete series, in particular) and one can reasonably expect that the subsequent results will generalize to other Lie groups. 


\section{The structure of the ground state sector}

For definiteness we consider from now on the case $G=\mathrm{SO}_{0}(1, N)$. The restricted dual then is a disjoint union $\widehat{G}_{r}=\widehat{G}_{d} \cup \widehat{G}_{p}$, of a set $\widehat{G}_{d}$ describing discrete UIR (unitary irreducible series representations) and a set $\widehat{G}_{p}$ describing principal series UIR. A UIR $\sigma_{d} \in \widehat{G}_{d}$ is parameterized a tuple of integers $\pm s \in \mathbb{N}, \xi \in \widehat{M}_{s} \subset \widehat{M}$, a UIR $\sigma_{p} \in \widehat{G}_{p}$ is labeled by a real parameter $\omega \geq 0$ and again by a tuple of integers $\xi \in \widehat{M}$. Here $\widehat{M}$ is the dual of the subgroup $\mathrm{SO}(N-2)$. We refer to appendices $\mathrm{A}$ and $\mathrm{B}$ for a brief survey of the relevant aspects of the representation theory of linear reductive Lie groups in general, and of $\mathrm{SO}_{0}(1, N)$ in particular.

Recall from Section 3 that the space of $\sigma$-equivariant ground states $\mathcal{G}_{\sigma}(\mathbf{T})$ can be isometrically identified with the eigenspace $\mathcal{E}_{\|\mathbf{T}\|}(\mathbf{T}(\sigma))$ of the fiber operator $\mathbf{T}(\sigma)=\mathbb{I} \otimes \check{\mathbf{T}}_{\check{\sigma}}$. The goal in the following is to successively rule out more and more UIRs $\sigma \in \widehat{G}_{r}$ for which the fiber spaces in $\mathcal{G}_{\sigma}(\mathbf{T})$ can be nonempty. This will lead to Theorems 1.1 and 1.2 , as announced in the introduction.

\section{1 $\mathcal{G}_{\sigma}(\mathbf{T})$ is empty for all but one principal series representation}

We begin by studying in more detail the restricted transfer operators $\mathbf{T}_{\omega \xi}:=\mathbf{T}_{\sigma}$, where we now write the label $\sigma$ as $(\omega, \xi)$ corresponding to a member of the principal series (see Appendix B). We also denote by $\widehat{M}$ the subset of $\widehat{G}_{r}$ corresponding to the principal series.

Here the realization of the model space $\mathcal{L}_{\sigma}$ as $L_{\xi}^{2}(K)$ and the realization of the infinite sums over $\kappa$ in terms of $k$-integrations is useful. See Appendix A.8. We first show that in this realization the function space $L_{\sigma}^{2}(\mathcal{N})_{0}$ in (2.39), for which we now write $L_{\xi}^{2}(\mathcal{N})$, takes the following form

$$
\begin{array}{ll}
f \in L^{2}\left(\mathcal{N} \times K, V_{\xi}\right), & \\
f\left(k_{0} n, k\right)=f\left(n, k k_{0}\right), & k_{0} \in K, \\
f(n, m k)=r_{\xi}(m) f(n, k), & m \in M .
\end{array}
$$

For fixed $n \in \mathcal{N}$ the second equation is just the defining relation for $L_{\xi}^{2}(K)$, see (A.43). The first relation is the realization of the equivariance condition under $\ell_{r}(K)$ in the compact model of the principal representation. Indeed the right action $f(n, k) \mapsto$ $f(n, k) \pi_{\omega \xi}(g)$ can be read off from Eq. (A.56b) and is given by

$$
f(n, k) \pi_{\omega \xi}(g)=(\delta \nu)^{-1}\left(a\left(k g^{-1}\right)\right) f\left(n, g^{-1}[k]\right) .
$$

For $g=k_{0} \in K$ the right hand side of (4.2) reduces to $f\left(n, k k_{0}^{-1}\right)$ so that the equivariance condition assumes the form given in (4.1). As a check one can verify the left $K$ invariance 


$$
f\left(k_{0} n, k\right) \pi_{\omega \xi}\left(k_{0} g\right)=f(n, k) \pi_{\omega \xi}(g), \quad k_{0} \in K,
$$

using $a\left(k g^{-1} k_{0}^{-1}\right)=a\left(k g^{-1}\right)$ and $k\left(k g^{-1} k_{0}^{-1}\right)=k\left(k g^{-1}\right) k_{0}^{-1}$ via (A.37).

The fiber operator $\mathbf{T}_{\omega \xi}$ will now act on the space of functions (4.1) as an integral operator with kernel

$$
\begin{aligned}
& \mathcal{T}_{\omega \xi}\left(n, n^{\prime} ; k, k^{\prime}\right):=\int_{G} d g \mathcal{T}\left(g, n^{\prime}, n\right)\left[\pi_{\omega \xi}(g)\right]\left(k^{\prime}, k\right) \\
& \quad=\int_{P} d p \mathcal{T}\left(k^{\prime-1} p k, n^{\prime}, n\right)\left(\delta^{-1} \nu\right)(p) r_{\xi}(m(p))=\mathcal{T}_{\omega \xi}\left(k n, k^{\prime} n^{\prime} ; e, e\right),
\end{aligned}
$$

using (A.54). Explicitly $\nu=\nu_{\omega}$ and $\delta$ are given by (B.8). From (3.4) and (A.54) one verifies the symmetries

$$
\mathcal{T}_{\omega \xi}\left(k_{0} n, n^{\prime} ; k k_{0}^{-1}, k^{\prime}\right)=\mathcal{T}_{\omega \xi}\left(n, n^{\prime} ; k, k^{\prime}\right)=\mathcal{T}_{\omega \xi}\left(n, k_{0} n^{\prime} ; k, k^{\prime} k_{0}^{-1}\right)
$$

which ensure that $\mathbf{T}_{\omega \xi}$ acts consistently on the function space (4.1). For fixed arguments the kernel (4.4) is a linear map on $V_{\xi}$ whose matrix elements obey

$$
\left|\left\langle v, \mathcal{T}_{\omega \xi}\left(n, n^{\prime} ; k, k^{\prime}\right) v^{\prime}\right\rangle_{V_{\xi}}\right| \leq \mathcal{T}_{0,0}\left(n, n^{\prime} ; k, k^{\prime}\right) \sup _{m \in M}\left|\left\langle v, r_{\xi}(m) v^{\prime}\right\rangle_{V_{\xi}}\right|
$$

for all $v, v^{\prime} \in V_{\xi}$. The inequality is strict unless $\omega=0$ and $\xi=0$. Here we wrote $\xi=0$ for the trivial representation of $M$ and $\omega=0$ refers to the $\omega \rightarrow 0$ limit of the principal series. The bound (4.6) is a manifest consequence of (4.4) and the unitarity of $\nu: A \rightarrow U(1)$. With these preparations we can show the crucial

Proposition 4.1. (a) The operators $\mathbf{T}_{\omega \xi}: L_{\xi}^{2}(\mathcal{N}) \rightarrow L_{\xi}^{2}(\mathcal{N})$ are bounded for all (not almost all) $\omega \geq 0, \xi \in \widehat{M}$. Their norms $\left\|\mathbf{T}_{\omega \xi}\right\|$ are continuous functions of $\omega$ and obey

$$
\left\|\mathbf{T}_{\omega \xi}\right\| \leq\left\|\mathbf{T}_{00}\right\| \quad \text { for all } \xi \in \widehat{M} \text { and } \omega \geq 0
$$

where the inequality is strict unless $\omega=0$ and $\xi=0$.

(b) $\mathbf{T}_{00}$ is a transfer operator in the sense of Definition 2.1 uniquely associated with $\mathbf{T}$.

Proof. (a) For the boundedness we show that the kernel of $\mathbf{T}_{\omega \xi}$ obeys the stronger condition

$$
\sup _{\|v\|=\left\|v^{\prime}\right\|=1} \sup _{n, k} \int d n^{\prime} d k^{\prime}\left|\left\langle v, \mathcal{T}_{\omega \xi}\left(n, n^{\prime}, k, k^{\prime}\right) v^{\prime}\right\rangle_{V_{\xi}}\right|<\infty .
$$

Clearly the expression under the first sup is bounded by

$$
\sup _{n} \int d g d n^{\prime} \mathcal{T}\left(g, n^{\prime}, n\right) \sup _{k, g_{0}} \int d k^{\prime}\left|\left\langle v, \pi_{\omega, \xi}\left(g_{0}\right)\left(k, k^{\prime}\right) v^{\prime}\right\rangle_{V_{\xi}}\right|
$$


The first factor is bounded by the constant $K_{\mathcal{T}}$ in (3.1). To estimate the second factor, let $\phi_{l}$ be a sequence of positive normalized functions on $G$ approximating a delta distribution centered around $g_{0} \in G$. From (A.54)

$$
\left|\left\langle v, \pi_{\omega \xi}\left(\phi_{l}\right)\left(k, k^{\prime}\right) v^{\prime}\right\rangle_{V_{\xi}}\right| \leq \int_{P} d p \delta(p)^{-1} \phi_{l}\left(k^{-1} p k^{\prime}\right) \sup _{m}\left|\left\langle v, r_{\xi}(m) v^{\prime}\right\rangle_{V_{\xi}}\right|
$$

The second factor is a finite constant and it suffices to consider the $d k^{\prime}$ integral of the first factor. Recall from Appendix A that $P=N A M, d p=d(n a) d m=\delta(a)^{2} d n d a d m$. Parameterizing $k^{\prime}$ as $k^{\prime}=k^{\prime}(\vec{s}) m^{\prime}$, with $m^{\prime} \in M, k^{\prime}(\vec{s}) \in K / M$ (see Appendix B) one has

$$
\int_{K} d k^{\prime} \int_{P} d(n a) d m \delta(a)^{-1} \phi_{n}\left(k^{-1} n a m k^{\prime}\right)=\int_{M} d m^{\prime} \int_{G} d g \delta(a(g)) \phi_{n}\left(k^{-1} g m^{\prime}\right) \leq 1,
$$

using $d g=\operatorname{dndadmdS}(\vec{s})$ from $(\underline{\mathrm{B} .5}),(\underline{\mathrm{B} .6})$, and the fact that $\delta(a(g)) \leq 1$. This gives (4.8). From here it follows (see Definition 2.1) that $\mathbf{T}_{\omega \xi}$ defines a bounded operator from $L_{\xi}^{p}(\mathcal{N})$ to $L_{\xi}^{p}(\mathcal{N})$, for all $1 \leq p \leq \infty$. For $p=2$ this is the assertion, for $p \neq 2$ it gives an alternative (though less general) proof of the extension discussed after Eq. (13.11).

To see the continuity of the norms, recall

$$
\left\|\mathbf{T}_{\omega \xi}\right\|=\sup _{f}\left|\left(f, \mathbf{T}_{\omega \xi} f\right)\right|, \quad f \in L_{\xi}^{2}(\mathcal{N}), \quad(f, f)=1
$$

We may assume that the supremum is reached on a sequence $\left(f_{l}\right)_{l \in \mathbb{N}}$ of normalized functions in $L_{\xi}^{2}(\mathcal{N})$, in which case it suffices to show that $\lim _{l \rightarrow \infty}\left(f_{l}, \mathbf{T}_{\omega \xi} f_{l}\right)$ is continuous in $\omega$. Each matrix element $\left(f_{l}, \mathbf{T}_{\omega \xi} f_{l}\right)$ is manifestly a continuous function of $\omega$. To show that this remains true for the limit $l \rightarrow \infty$ we establish a uniform bound: using (4.1), (4.4) one has for the matrix elements

$$
\begin{aligned}
\left(f_{l}, \mathbf{T}_{\omega \xi} f_{l}\right)= & \int d n d n^{\prime} d k d k^{\prime} \int_{P} d p \mathcal{T}\left(k^{\prime-1} p k, n^{\prime}, n\right)\left(\delta^{-1} \nu\right)(p) \\
& \times\left\langle f_{l}(n, k), r_{\xi}(m(p)) f_{l}\left(n^{\prime}, k^{\prime}\right)\right\rangle_{V_{\xi}},
\end{aligned}
$$

where $\omega$ enters only through the phase $\nu(p)=\nu_{\omega}(p)$, see (B.8). The modulus of these matrix elements is easily seen to have the $l$ and $\omega$ independent upper bound

$$
\sup _{n, k, n^{\prime}, k^{\prime}} \int_{P} d p \mathcal{T}\left(k^{\prime-1} p k, n^{\prime}, n\right) \delta(p)^{-1}<\infty .
$$

By dominated convergence one can take the $l \rightarrow \infty$ limit inside the integrals, after which continuity in $\omega$ is manifest. 
Finally, to verify (4.7) we return to (4.12). From (4.6) the modulus of the matrix elements can be bounded by

$$
\begin{aligned}
& \left|\left(f, \mathbf{T}_{\omega \xi} f\right)\right| \leq \int d k d n d k^{\prime} d n^{\prime}\left|\left\langle f(n, k), \mathcal{T}_{\omega \xi}\left(n, n^{\prime} ; k, k^{\prime}\right) f\left(n^{\prime}, k^{\prime}\right)\right\rangle_{V_{\xi}}\right| \\
& \leq \int d k d n d k^{\prime} d n^{\prime} \mathcal{T}_{0,0}\left(n, n^{\prime} ; k, k^{\prime}\right)\|f(n, k)\|_{V_{\xi}}\left\|f\left(n^{\prime}, k^{\prime}\right)\right\|_{V_{\xi}},
\end{aligned}
$$

where the second inequality is strict unless $\omega=0$ and $\xi=0$. Here both norms are pointwise nonnegative functions in $L_{0}^{2}(\mathcal{N})$. In fact one gets a norm preserving map from $L_{\xi}^{2}(\mathcal{N})$ to a subset of $L_{0}^{2}(\mathcal{N})$ by mapping $f(n, k)$ to $f_{0}(n, k):=\|f(n, k)\|_{V_{\xi}}$. In particular $f$ has unit norm in $L_{\xi}^{2}(\mathcal{N})$ if and only if $f_{0}$ has unit norm in $L_{0}^{2}(\mathcal{N})$.

On the other hand to get the norm of $\mathbf{T}_{00}$ the supremum of $\left(f_{0}, \mathbf{T}_{00} f_{0}\right)$ over all normalized $f_{0} \in L_{0}^{2}(\mathcal{N})$ has to be taken. These are complex valued functions but by the integral representation (4.4) $\mathcal{T}_{00}\left(n, n^{\prime}, k, k^{\prime}\right) \geq 0$ and the supremum is reached on the subset of (all) nonnegative functions in $L_{0}^{2}(\mathcal{N})$. Among those are the ones in the range of the map $L_{\xi}^{2}(\mathcal{N}) \rightarrow L_{0}^{2}(\mathcal{N}), f \mapsto\|f\|_{V_{\xi}}$, described above.

So one has for $(\xi, \omega) \neq(0,0)$

$$
\left\|\mathbf{T}_{\omega \xi}\right\| \leq \sup _{f \in L_{\xi}^{2}(\mathcal{N})}\left|\left(f, \mathbf{T}_{\omega \xi} f\right)\right|<\sup _{f}\left(|f|, \mathbf{T}_{00}|f|\right) \leq \sup _{f_{0} \in L_{0}^{2}(\mathcal{N})}\left(f_{0}, \mathbf{T}_{00} f_{0}\right)=\left\|\mathbf{T}_{00}\right\|
$$

(b) From part (a) we know that $\mathbf{T}_{00}$ is a bounded linear integral operator with spectrum contained in $[0,\|\mathbf{T}\|]$. The integral representation (4.4) shows that its kernel is strictly positive almost everwhere. Thus $\mathbf{T}_{00}$ is also positivity improving and by (4.8) it is a transfer operator in the sense of Definition 2.1.

As a corollary we obtain part (b) of Theorem 1.1 announced in the introduction.

Corollary 4.1. $\mathcal{G}_{\sigma}(\mathbf{T})$ is non-empty for at most one principal series representation - the limit of the spherical principal representation, $\sigma=(\omega=0, \xi=0)$. Further $\mathcal{G}_{00}(\mathbf{T})$ can be isometrically identified with the ground state sector $\mathcal{G}\left(\mathbf{T}_{00}\right)$ of the transfer operator $\mathbf{T}_{00}$.

We add some comments on the discrete series. Recall from (3.20)

$$
\|\mathbf{T}\|=\operatorname{ess} \sup _{\sigma \in \widehat{G}_{r}}\left\|\mathbf{T}_{\sigma}\right\|
$$

and that a discrete series UIR is labeled by a tuple of integers $\pm s \in \mathbb{N}, \xi \in \widehat{M}_{s} \subset \widehat{M}$.

Remark 4.1. We expect that a preferred class of discrete series representations exists such that a bound similar to (4.7) holds. If there are discrete series UIR for which 
$\left\|\mathbf{T}_{ \pm s \xi}\right\|=\|\mathbf{T}\|$, a natural conjecture is that they have the minimal possible $M$ weight for a given $s \in \mathbb{N}$, i.e. $\xi=\xi_{s}:=(|s|, \ldots,|s|)$. This requires that the norms $\left\|\mathbf{T}_{ \pm s \xi}\right\|$ of the operators $\mathbf{T}_{ \pm s \xi}$ obey

$$
\left\|\mathbf{T}_{ \pm s \xi}\right\| \leq\left\|\mathbf{T}_{ \pm s, \xi_{s}}\right\| \quad \text { for all } \xi \in \widehat{M}_{s}
$$

where the inequality is strict unless $\xi=\xi_{s}:=(|s|, \ldots,|s|)$.

A verification would require a concrete model for the discrete series (like that of the principal series described in Appendix A.8) producing a counterpart of Eq. (4.4). We leave this for a future investigation. Here we proceed by identifying simple conditions under which discrete series representations are ruled out as candidates for the representation carried by $\mathcal{G}_{\sigma}(\mathbf{T})$. A set of such conditions has already been identified in corollary 3.1. We proceed by studying the role of positivity.

\subsection{The role of strict positivity}

One property which one expects from a ground state is "not to have nodes". This means the ground state wave function can be chosen strictly positive a.e. In this section we show that, somewhat surprisingly, the positivity requirement uniquely determines the representation carried by $\sigma$-equivariant eigenfunctions.

As before we denote by $\pi_{00}$ the limiting representation of the spherical principal series $\pi_{\omega 0}, \omega \geq 0$, of $G=\mathrm{SO}_{0}(1, N)$, and by $\mathbf{A}_{00}$ the corresponding fiber operator in the decomposition of $\mathbf{A}$ (see Section 3 ). Further we set

$$
\mathcal{P}=\left\{\Omega \in L^{\infty, 2}\left(\mathcal{M}_{r}\right) \text { or } \Omega \in M^{-1} L^{\infty, 2}\left(\mathcal{M}_{r}\right) \mid \Omega(g, n)>0 \text { a.e. }\right\}
$$

for the settings in Sections 3.3 or 3.4, respectively.

Proposition 4.2. $\mathcal{E}_{\lambda, \sigma}(\mathrm{A}) \cap \mathcal{P}=\{0\}$ unless $\pi_{\sigma}=\pi_{00}$. Further the intersection is isometric to $\mathcal{E}_{\|\mathbf{A}\|}\left(\mathbf{A}_{00}\right)$ and is spanned by a.e. strictly positive functions.

Proof. Recall from Section 3 that the generalized eigenspaces $\mathcal{E}_{\lambda, \sigma}(\mathbf{A})$ invariant under $\pi_{\sigma}$ are generated by 'factorized' functions of the form $\sum_{i} \tau_{v_{i} \sigma}\left(\chi_{i}\right)(g, n)$. Here

$$
\tau_{v \sigma}(\chi)(g, n)=\left(\chi(n), \pi_{\sigma}(g) v\right)_{\sigma}=\sum_{\kappa s} \chi(n)_{\kappa s}^{*}\left(\pi_{\sigma}(g) v\right)_{\kappa s}
$$

with $v \in \mathcal{L}_{\sigma}$ and $\chi \in \mathcal{E}_{\lambda}\left(\mathbf{A}_{\sigma}\right)$ a generalized eigenfunction of $\mathbf{A}_{\sigma}$. By assumption at least one such function is strictly positive a.e. on $\mathcal{M}_{r}$, for which we write $\Omega(g, n)=$ $\sum_{i}\left(\chi_{i}(n), \pi_{\sigma}(g) v_{i}\right)_{\sigma}$. Replacing $g$ with $k g, k \in K$, and averaging over $K$ must still 
produce an a.e. strictly positive function. On the other hand, evaluated in the $K$ adapted basis the average reads

$$
\int_{K} d k \Omega(k g, n)=\sum_{i} \chi_{i}(n)_{00}^{*} P_{0} \pi_{\sigma}(g) v_{i}>0
$$

Importantly the $K$ average cannot vanish, so at least one of the projections $P_{0} \pi_{\sigma}(g) v_{i}$ must be nonzero. This means $\mathcal{L}_{\sigma}$ must contain a $K$-invariant vector, namely $P_{0} \pi_{\sigma}(g) v_{i}$ for some $i$, and $\pi_{\sigma}$ is by definition of $K$-type 1 . But for the UIR of $\mathrm{SO}_{0}(1, N)$ this is the case only for $\pi_{\omega, \xi=0}$, see Appendix B. Discrete series representations and non-spherical principal series representations are ruled out. Thus, for $\mathcal{E}_{\lambda, \sigma}(\mathbf{A}) \cap \mathcal{P}$ to be non-empty the corresponding UIR $\pi_{\sigma}$ must be a member of the spherical principal series, $\pi_{\sigma}=\pi_{\omega 0}$, for some $\omega \geq 0$.

It remains to show that only the $\omega \rightarrow 0$ limit can occur. To this end we replace $g$ in (4.21) by $g k$ and average over $K$. Using the fact that $\pi_{\omega, 0}(g)_{00,00}$ evaluates to the Legendre function $E_{\omega, 00}\left(q_{0}\right), q=g^{-1} q^{\uparrow}=\left(q_{0}, \vec{q}\right)$, in (B.23) one gets the condition

$$
\int_{K \times K} d k d k^{\prime} \Omega\left(k g k^{\prime}, n\right)=\sum_{i} \chi_{i}(n)_{00}^{*}\left(v_{i}\right)_{00} E_{\omega, 00}\left(q_{0}\right)>0,
$$

for almost all $n \in \mathcal{N}$ and $q_{0}>1$. However, $E_{\omega, 00}$ is an oscillating function of its argument unless $\omega=0$. This means (4.22) cannot be satisfied for $\omega \neq 0$.

We remark that the first part of the argument extends to all Lie groups of the type considered: $\mathcal{E}_{\lambda, \sigma}(\mathbf{A}) \cap \mathcal{P}=\{0\}$ unless $\pi_{\sigma}$ is of $K$-type 1 . The second part of the argument shows that $\pi_{\sigma}$ must have a $K$-spherical function $p(g)=\pi_{\sigma}(g)_{00,00}$ which is real and positive on all of $G$, i.e. on $K \backslash G / K$. An interesting (apparently open) question is which nonamenable Lie groups have a UIR with a pointwise positive $K$-spherical function (not just one of positive type).

As a corollary we now obtain part (a) of Theorem 1.1 as anticipated in the introduction.

Corollary 4.2. $\mathcal{G}_{\sigma}(\mathbf{T})$ is empty for all but the principal series representations whenever one of the following holds: (i) $\mathcal{G}_{\sigma}(\mathbf{T})$ contains a strictly positive function. (ii) $\mathcal{G}_{\sigma}(\mathbf{T})$ contains a $K$-singlet. (iii) $\mathbf{T}_{\sigma}$ is compact.

Proof. (i) is a special case of Proposition 4.2. (ii) and (iii) follow from Corollary 3.1.

Combined parts (a) and (b) of Theorem 1.1 show that under broad conditions the representation $\sigma$ carried by the equivariant fibers $\mathcal{G}_{\sigma}(\mathbf{T})$ of the ground state sector is uniquely determined and always the same for every invariant transfer operator, namely the limit of the spherical principal series. The existence of generalized ground states in 
$\mathcal{G}_{00}(\mathbf{T}) \simeq \mathcal{G}\left(\mathbf{T}_{00}\right)$ is however not guaranteed from the outset. A simple but important special case where it is, is when all the fiber operators $\mathbf{T}_{\sigma}$ are compact.

\section{$4.3 \mathcal{G}_{\sigma}(\mathbf{T})$ for $\mathbf{T}_{\sigma}$ compact}

Here we present the proof of Theorem 1.2, as anticipated in the introduction. We reformulate the theorem in a more precise way as follows:

Theorem 1.2.' Let $\mathbf{T}$ be an invariant transfer operator in the sense of Definition 2.1 and 3.1, and assume that its kernel satisfies in addition the condition (C) in Section 3.3. Then all its ground state fibers $\mathcal{G}_{\sigma}(\mathbf{T})$ but $\mathcal{G}_{00}(\mathbf{T}) \simeq \mathcal{E}_{\|\mathbf{T}\|}\left(\mathbf{T}_{00}\right)$ are empty. Further $\mathbf{T}_{00}$ is a transfer operator whose ground state sector $\mathcal{G}\left(\mathbf{T}_{00}\right)=\mathcal{E}_{\|\mathbf{T}\|}\left(\mathbf{T}_{00}\right)$ is nonempty and is spanned by a unique normalizable and a.e. strictly positive function $\psi_{0} \in L^{2}\left(\mathcal{N} \times S^{N-1}\right) \simeq$ $L^{2}(\mathcal{N}) \otimes \mathcal{L}_{00}$. Explicitly $\mathcal{G}(\mathbf{T})$ is the linear hull of functions

$$
\Omega(g, n)=\int_{S^{N-1}} d S(p) \frac{\psi_{0}(n, \vec{p})}{\left(q_{0}-\vec{q} \cdot \vec{p}\right)^{\frac{N-1}{2}}}
$$

where $q=g q^{\uparrow}=\left(q_{0}, \vec{q}\right)$.

Proof. By Theorem 1.1 we know that at most $\mathcal{G}\left(\mathbf{T}_{00}\right)$ is nonempty. All functions in $\mathcal{G}\left(\mathbf{T}_{00}\right)$ are thus $M$-singlets and take values in $\mathbb{C}=V_{\xi=0}$. By Lemma 3.2 and Proposition $4.1 \mathbf{T}_{00}$ is a Hilbert-Schmidt operator and a transfer operator in the sense of Definition 2.1. Its eigenspace $\mathcal{E}_{\|\mathbf{T}\|}\left(\mathbf{T}_{00}\right)$ coincides with its ground state sector $\mathcal{G}\left(\mathbf{T}_{00}\right)$. By the generalized Perron-Frobenius theorem [19, 44] it follows that the ground state sector of $\mathbf{T}_{00}$ is one-dimensional (non-empty, in particular) and is spanned by a unique a.e. strictly positive function $\psi_{0}(n, k)>0$ on $\mathcal{N} \times K$, with $\psi_{0}\left(k_{0} n, k\right)=\psi_{0}\left(n, k k_{0}\right)$, namely the ground state of $\mathbf{T}_{00}$. Note that via the realization (4.1) the result is applied to scalar valued functions on $\mathcal{N} \times K$ rather than to $\mathcal{L}_{00}$-valued functions on $\mathcal{N}$. Since $\xi=0$ the function also obeys $\psi_{0}(n, m k)=\psi_{0}(n, k)$, for all $m \in M$, and thus can be viewed as a function on $\mathcal{N} \times S^{N-1}$, with $S^{N-1}=M \backslash K$. This is related to the fact that the $K$-content of the principal series representations $\pi_{\omega, \xi}$ with $\xi=0$ equals $\widehat{K}_{\xi=0}=\{(0, \ldots, 0, \ell), \ell \geq$ $0\}$. These are the $\mathrm{SO}(N)$ irreducible representations which can be realized on the space of symmetric traceless tensors, or equivalently on the harmonic symmetric polynomials of degree $\ell$ on the sphere $S^{N-1}$. In the original model, where $\psi_{0}: \mathcal{N} \rightarrow \mathcal{L}_{00}$, we relabel the components $\psi_{0}(n)_{\kappa s}$ in the $K$-adapted basis such that $\psi_{0}(n)_{\kappa s}=\psi_{0}(n)_{\ell m}$, where $\psi_{0}(n)_{\ell m}$ are the components of $\psi_{0}(n, \vec{p})$ in a basis of spherical harmonics $Y_{\ell m}(\vec{p})$ on $S^{N-1}$. The matrix element $\tau_{v, 00}\left(\psi_{0}\right)(g, n)=\left(\psi_{0}(n), \pi_{00}(g) v_{0}\right)$, then evaluates to a double sum over labels of the spherical harmonics. This holds for any reference vector $v \in \mathcal{L}_{00}$. Specifically we choose now for $v$ the unique $K$-invariant vector $v_{0} \in \mathcal{L}_{00}$, i.e. the vector satisfying $\pi_{00}(k) v_{0}=v_{0}$ for all $k \in K$. In the $K$-adapted basis of $\mathcal{L}_{00}$ this vector 
has components $\left(v_{0}\right)_{\kappa s}=\left(v_{0}\right)_{00} \delta_{\kappa=0} \delta_{s=0}$. The matrix element then evaluates to

$$
\tau_{v_{0}, 00}\left(\psi_{0}\right)(g, n)=\left(\psi_{0}(n), \pi_{00}(g) v_{0}\right)=\sum_{\ell m} \psi_{0}(n)_{\ell m} \pi_{00}(g)_{\ell m, 00}\left(v_{0}\right)_{00} .
$$

The matrix elements $\pi_{00}(g)_{\ell m, 00}$ are right $K$ invariant and reduce to functions on $Q=$ $G / K$, with the identification $q=g q^{\uparrow}$. In the case at hand these are the Legendre functions $E_{\omega=0, \ell m}$ described in (B.24). Using the integral representation in (B.24) the sum can be replaced by an integral over $S^{N-1}: \sum_{\ell m} \psi_{0}(n)_{\ell m} E_{0, \ell m}(q)=\int d S(p) \epsilon_{0, p}(q) \psi_{0}(n, \vec{p})$, with $\psi_{0}(n, \vec{p})=\sum_{\ell m} \psi_{0}(n)_{\ell m} Y_{\ell m}(\vec{p})$. Inserting (B.18) and omitting the overall constant $\left(v_{0}\right)_{00}$ gives the announced expression (4.23).

It remains to show that the linear hull of these functions is dense in $\mathcal{G}(\mathbf{T})$. We proceed in two steps. First we show that the choice of the $K$-invariant vector $v_{0}$ was inessential. This is because on account of the irreducibility of $\mathcal{L}_{00}$ every vector $v \in \mathcal{L}_{00}$ lies in the closure of linear combinations of the form $\sum_{i} c_{i} \pi_{00}\left(g_{i}\right) v_{0}$. Thus $\tau_{v, 00}(g, n)$ with $v \in \mathcal{L}_{00}$ generic lies in the linear hull of the functions (4.24). In a second step we appeal to Proposition 3.6, according to which linear combinations of the form (3.43) (but now with a unique $\left.\chi_{\lambda=\|\mathbf{T}\|, \sigma=00, r=1}=\psi_{0}\right)$ are dense in $\mathcal{E}_{\lambda=\|\mathbf{T}\|, \sigma=(00)}(\mathbf{T})=\mathcal{G}(\mathbf{T})$.

Remark 4.2. Theorem 1.2' is a generalization of the result announced in Section 3.3 of [12]; as a special case it contains the omitted proof of (a slightly corrected variant of) Theorem 2 in [12]. Explicitly: Let $\mathbb{T}$ be the transfer operator of the hyperbolic nonlinear sigma-model (see Eqs. (2.3),(2.4) of [12]). Then $\mathbb{T}^{2}$ satisfies the premise of Theorem 1.2.

Proof. One only has to check that the condition (C) is satisfied. This follows from the Lemma in Section 3.3 of $[12$.

\section{Conclusions and outlook}

The set of ground states of a quantum mechanical many particle system - equivalently, a statistical mechanics system on a finite lattice - whose dynamics is invariant under a nonamenable unitary representation of a locally compact group has been shown here to have a remarkable structure: despite the invariance of the dynamics there are always infinitely many noninvariant and nonnormalizable ground states. This spontaneous symmetry breaking cannot be prevented by tuning a coupling or temperature parameter, and it takes place even in one and two dimensions and even for finite systems. The phenomenon is also not limited to a semiclassical regime. The linear space spanned by these ground states carries a distinguished unitary irreducible representation of the 
group, which for $\mathrm{SO}_{0}(1, N)$ has been identified as the limit of the spherical principal series. These properties hold for a large class of transfer operators or Hamiltonians. Compactness of the transfer operators restricted to the group invariant fibers is a sufficient but by no means necessary condition. The same is true for the very existence of generalized ground states.

As explained in Section 2.1 the well-known theory of generalized eigenfunctions due to Gel'fand [17] and their application to group representations due to Maurin [34 is not sufficient to assure the existence of generalized ground states (neither as functions nor as distributions). However, we can guarantee their existence as functions in various situations considerably broader than the above one. These involve by neccessity a subsidiary condition on the transfer operator. One can take [39] (s1) $\mathbf{T}_{00}$ is a compact operator. (s2) the integral kernel of $\mathbf{T}$ has an extremizing configuration $m_{*} \in \mathcal{M}$, in the sense that $T\left(m, m^{\prime} ; t\right) \leq T\left(m_{*}, m_{*}, t\right)$ for all $m, m^{\prime} \in \mathcal{M}$, and possibly others. In these cases one has the following construction principle:

Theorem 5.1. Let $\mathbf{T}$ be as in Theorem 1.1 and such that the subsidiary condition (s1) or (s2) is satisfied. Set $\Omega_{t}(v):=\mathbf{T}^{t} v /\left(v, \mathbf{T}^{t} v\right)$ for $v \in L^{2}(\mathcal{M})$ and $t \in \mathbb{N}$. Then there exist (explicitly known) vectors $v_{*} \in L^{2}(\mathcal{M})$ and a sequence of integers $\left(t_{j}\right)_{j \in \mathbb{N}}$ such that the limit

$$
\Omega_{\infty}:=w^{*}-\lim _{j \rightarrow \infty} \Omega_{t_{j}}\left(v_{*}\right)
$$

exists and is an element of $\mathcal{G}(\mathbf{T})$.

The point of Theorem 5.1 is that even a weak limit has the tendency to either diverge or to vanish. It is only by a carefully tuned 'renormalization' that a finite and nonzero limit can be obtained which is a generalized ground state of $\mathbf{T}$. The choice of the sequence $t_{j}$ is presumably inessential, but the choice of specific vectors $v_{*} \in L^{2}(\mathcal{M})$ is crucial. Typically these 'seed' vectors form a set of measure zero in $L^{2}(\mathcal{M})$ and their identification is a major part of the Theorem.

The results for these 'finite' systems can be generalized in a number of ways. The choice of $\mathcal{M}$ as a product manifold $\mathcal{M}=Q^{\times \nu}$ is natural from the viewpoint of a quantum mechanical or statistical mechanics interpretation. One could also replace $\mathcal{M}$ with the phase space of a dynamical system with constraints generating the Lie algebra of a nonamenable group, in which case $\mathcal{M}$ could be any proper riemannian $G$ manifold (in the sense of [35]). We expect that the results of sections 2 and 3 remain valid in this more general setting. The main reason for specializing to $G=\mathrm{SO}_{0}(1, N)$ in Section 4 was that these groups have split rank 1, so their restricted dual is relatively simple. Nevertheless we expect that counterparts of Theorem 1.1 exists for basically every noncompact semi-simple Lie group with a finite center: only a tiny subset of unitary irreducible representations are potential candidates for the representation carried by the ground state sector of a system with a $G$ invariant dynamics.

Another extension is to supersymmetric multiparticle systems with nonamenable symmetries (see 8] for one-particle systems). The transfer operators associated with graded 
homogeneous spaces are relevant for the description of disordered systems [13, 14, 63. They are not symmetric with respect to a positive definite scalar product, but have a unique ground state in a certain sense. Restricted to a bosonic subspace transfer operators related to the ones considered here arise. This leads to the expectation that the lowest lying excitations of the original system would have a structure analogous to that of the ground state space found here.

The analysis of the thermodynamic limit is an open problem. In the operator/Hilbert space formulation the thermodynamic limit can presumably at best be taken for specific lattices (hierarchical, Cayley trees, etc). For the hypercubic lattices directly relevant to lattice quantum field theories one needs to analyze the limit in terms of correlation functions, see 49]. Expectations are however that the structure of the ground state sector, if it changes, will 'fragment' even more [38, 12]. In fact the existence of non-invariant expectation values in the thermodynamic limit is a more-or-less direct consequence of the non-amenability [12]. This should lead to a general no-go theorem (inverse, nonconstructive Coleman theorem).

We believe that this circle of ideas will have a variety of applications, ranging from localization phenomena in solid state physics and quantum Kaluza-Klein theories to AdS duality and the ground states of quantum gravity. We hope to return to these applications elsewhere.

Acknowledgments: We wish to thank M. Zirnbauer for calling our attention to the transfer operators on graded homogeneous spaces. E. S. would like to thank H. Saller for useful discussions about abstract harmonic analysis. 


\section{Appendix A: Harmonic analysis on noncompact Lie groups}

In this appendix we summarize the results on the representation theory and the harmonic analysis of non-compact Lie groups needed in the main text. In appendix B we present more explicit results for $\mathrm{SO}(1, N)$.

1. Basic setting: The harmonic analysis on a noncompact Lie group is governed by Plancherel-type decompositions

$$
L^{2}(G)=\int_{\widehat{G}_{r}}^{\oplus} d \nu(\sigma) \mathcal{L}_{\sigma}(G) \otimes \check{\mathcal{L}}_{\check{\sigma}}(G), \quad \rho \times \ell=\int_{\widehat{G}_{r}}^{\oplus} d \nu(\sigma) \pi_{\sigma} \otimes \pi_{\check{\sigma}},
$$

where $d \nu$ is the Plancherel measure on $\widehat{G}$ which has support only on a proper subset $\widehat{G}_{r} \subset$ $\widehat{G}$, the restricted dual of the group $G$. The $\pi_{\sigma}$ are unitary continuous representations and $\mathcal{L}_{\sigma}:=\mathcal{L}_{\sigma}(G)$ is irreducible for $\nu$ almost all $\sigma \in \widehat{G}_{r}$. In the following we present the precise version of (A.1) used in the main text and set the notation.

We use the variant of (A.1) valid for any linear connected reductive Lie group $G$ (linear meaning that $G$ is a closed subgroup of $\operatorname{GL}(N, \mathbb{R})$ or $\operatorname{GL}(N, \mathbb{C})$, reductive meaning that it is stable under conjugate transpose). Examples are $\mathrm{SO}(1, N), \mathrm{SO}(N, \mathbb{C}), \mathrm{SL}(N, \mathbb{R})$, $\mathrm{SU}(m, n), \operatorname{Sp}(2 N, \mathbb{R})$. The Plancherel measure $d \nu(\sigma)$ is then a combination of a counting measure for discrete variables and a Lebesgue measure for continuous variables (see 25] and references therein); for groups $G$ having no compact Cartan subgroup the discrete part is absent (by a theorem of Harish-Chandra, see e.g. 53]). This holds in particular for the complex connected simple Lie groups [33, 55]. The spaces $\mathcal{L}_{\sigma}$ are Hilbert spaces equipped with an inner product $(,)_{\sigma}$ depending on $\sigma$. As a consequence of the multiplicity theorems decribed below one can identify each $\mathcal{L}_{\sigma}$ with a subspace of $L^{2}(K)$, where $K$ is a maximal compact subgroup of $G$. The $\pi_{\sigma}$ are distributions over a space of test functions $\mathcal{D}$ on $G$ which take values in the trace class operators on $\mathcal{L}_{\sigma}$. For the class of Lie groups considered the distributions arise by integration with respect to a $L^{\infty}(G)$ function $\pi_{\sigma}(g)$. Thus, for each $\phi \in \mathcal{D}$ the integral $\pi_{\sigma}(\phi):=\int d g \phi(g) \pi_{\sigma}(g)$ (where $d g$ is Haar measure on $G$ ) is a trace class operator whose matrix elements in some orthonormal basis $e_{i}$ in $\mathcal{L}_{\sigma}$ we denote by $\int d g \phi(g) \pi_{\sigma}(g)_{i j}$. A typical choice for $\mathcal{D}$ is $\mathcal{C}_{c}^{\infty}(G)$, the smooth compactly supported functions on $G$. For $\psi \in L^{2}(G) \cap L^{1}(G)$ the operator $\pi_{\sigma}(\psi)$ is Hilbert-Schmidt, for $\psi \in L^{1}(G)$ it is still compact [18, 15]. The $\mathbb{C}$-valued functions $g \mapsto \pi_{\sigma}(g)_{i j}$ are called the coefficients of $\pi_{\sigma}$. They are continuous functions but in general do not have sufficient decay to be (square) integrable on $G$. This weak continuity is equivalent $\left(\left[15\right.\right.$, p.68) to the continuity of the $\mathcal{L}_{\sigma}$-valued functions.

The Plancherel theorem states that $\phi \in \mathcal{D}$ can be expanded in terms of eigenfunctions 


$$
\begin{aligned}
\phi(g) & =\int_{\widehat{G}_{r}} d \nu(\sigma) \sum_{i, j} \widehat{\phi}(\sigma)_{i j} \pi_{\sigma}(g)_{i j}, \\
\widehat{\phi}(\sigma)_{i j} & =\int_{G} d g \phi(g) \pi_{\sigma}\left(g^{-1}\right)_{i j} .
\end{aligned}
$$

The Plancherel-Parseval identity

$$
\int_{G} d g \phi(g)^{*} \psi(g)=\int_{\widehat{G}_{r}} d \nu(\sigma) \sum_{i j} \widehat{\phi}(\sigma)_{i j}^{*} \widehat{\psi}(\sigma)_{i j},
$$

expresses the unitarity of the map $\phi \mapsto \widehat{\phi}(\sigma)$.

The irreducible representations on which the Plancherel measure has support turn out to come in families parameterized by conjugacy classes of Cartan subgroups of $G$. The Cartan subgroups are of the form $H=T \times R$, where $T$ is compact and $R \simeq \mathbb{R}^{d}$ for some $d$. Its dual $\widehat{H}$ has discrete parameters coming from $\widehat{T}$ and continuous parameters coming from $\widehat{R}$. The labels $\sigma$ should be thought of as elements of $\widehat{H}$ and the Plancherel measure takes the form

$$
\int_{\widehat{G_{r}}} d \nu(\sigma)=\sum_{H} \int_{\widehat{H}} d \sigma \nu(H: \sigma),
$$

where the sum extends over the (finite number of) conjugacy classes of Cartan subgroups of $G$ and the functions $\nu(H: \sigma)$ are known (25] and references therein). A Cartan subgroup here is defined as a maximal abelian subalgebra all of whose elements are diagonalizable as matrices over the complex numbers. The representations $\sigma \in \widehat{G}_{r}$ associated with a compact Cartan subgroup are called discrete series representations, all others are called (on the basis of a theorem) cuspidial principal series. Among them are the principal series proper associated with a unique conjugacy class to be described below. For example $\mathrm{SL}(2, \mathbb{R})$ has two conjugacy classes of Cartan subgroups, $H=\mathrm{SO}(2)$ and $H=\left\{\operatorname{diag}\left(a, a^{-1}\right) \mid a \in \mathbb{R} \backslash\{0\}\right\}$. In contrast, $\mathrm{SL}(n, \mathbb{R})$ with $n>2$ has $[n / 2]+1$ conjugacy classes of Cartan subgroups, none of which is compact. The case $G=\mathrm{SO}_{0}(1, N)$ will be discussed in more detail in Appendix B.

2. Cartan subgroups: The structure of the Cartan subgroups $H$ entering (A.4) is best described in terms of their Lie algebra $\mathfrak{h}$. For the Lie groups considered there exists an involution $\iota$ such that the set of its fixed points generates a maximal compact subgroup $K$ of $G$. (More precisely $\left(G_{\iota}\right)_{0} \subset K \subset G_{\iota}$, where $G_{\iota}$ is the fixed point set and $\left(G_{\iota}\right)_{0}$ is its identity component). The involution $\iota$ of $G$ induces a decomposition of the Lie algebra $\mathfrak{g}=\mathfrak{k} \oplus \mathfrak{q}$, where $\mathfrak{k}$ and $\mathfrak{q}$ are even and odd under (the differential of) $\iota$, respectively. The Lie algebras $\mathfrak{h}$ can be assumed to be invariant under $\iota$. They are of the form $\mathfrak{h}=\mathfrak{k}_{0}+\mathfrak{a}_{0}$, 
where $\mathfrak{k}_{0} \subset \mathfrak{k}, \mathfrak{a}_{0} \subset \mathfrak{a}$. Here $\mathfrak{a}$ is the Lie algebra of the subgroup $A$ in the Iwasawa decomposition $G=N A K$. To $\mathfrak{k}_{0}, \mathfrak{a}_{0}$ one can associate a subgroup $P_{0}=N_{0} A_{0} M_{0}$ of $G$ called a "cuspidial parabolic subgroup". Here $N_{0}$ is a nilpotent subgroup of $N, A_{0}$ is a subgroup of $A$, and $M_{0}$ is such that $A_{0} M_{0}$ is the centralizer of $A_{0}$ in $G$. There is a systematic technique, called parabolic induction, which allows one to construct unitary representations of $G$ from those of $P_{0}$. Almost all of them are irreducible and provide the above "cuspidial principal series" representations. For our purposes the most important one is the principal series proper, which is associated with the (up to conjugacy) unique Cartan subgroup $H$ for which $\mathfrak{h} \cap \mathfrak{q}=\mathfrak{a}$, i.e. for which $A_{0}$ is all of $A$. The associated subgroup $P=N A M$ is called minimal parabolic and the construction of the associated principal series representations will be detailed later.

The other extreme case is when $A_{0}=\{e\}$ consists of the identity only, in which case $P_{0}$ is all of $G$. The associated representations are precisely the above discrete series representations. By a theorem of Harish-Chandra $G$ has discrete series representations if and only it has a compact Cartan subgroup. Equivalently ([53], p.282)

$$
\operatorname{rank} K=\operatorname{rank} M+\operatorname{dim} A
$$

where $\operatorname{rank} K$ is the dimension of the maximal torus of $K$. For example all $\mathrm{SO}_{0}(p, q)$ groups with $p q$ even have discrete series, as do $\mathrm{Sp}(n, \mathbb{R}), \mathrm{SU}(p, q)$, and $\mathrm{SL}(2, \mathbb{R})$. Groups which do not have discrete series are $\mathrm{SL}(n, \mathbb{R}), n>2$, and $\mathrm{SO}_{0}(p, q)$ with $p q$ odd. Several constructions of (all) discrete series representations are known, see [46]. In particular there is an elegant construction based on the kernel of the Dirac operator on homogeneous spinor bundles, see [41, 1].

The content of the theorem mentioned after (A.4) is that the restricted dual $\widehat{G}_{r}$ is exhausted by the above cuspidial principal series representations and the discrete series representations.

3. Tensor product conventions: Let $\mathcal{H}$ be a separable Hilbert space with inner product $($,$) , linear in the right and anti-linear in the left argument. The conjugate Hilbert$ space $\check{\mathcal{H}}$ is the Hilbert space with underlying additive group identical to that of $\mathcal{H}$ but with scalar multiplication defined by $(\lambda, \check{v}) \mapsto \lambda^{*} \check{v}$, for $\lambda \in \mathbb{C}, \check{v} \in \check{\mathcal{H}}$, and inner product $(\check{v}, \check{w}):=(w, v)$. The Hilbert space $\check{\mathcal{H}}$ can canonically be identified with the space of linear forms $\mathcal{L}(\mathcal{H}, \mathbb{C})$ on $\mathcal{H}$. Indeed by Riesz theorem $\mathcal{L}(\mathcal{H}, \mathbb{C}) \ni(v, \cdot) \mapsto \check{v} \in \check{\mathcal{H}}$ is a linear isomorphism of complex vector spaces. The map $\mathcal{H} \rightarrow \mathscr{\mathcal { H }}$ is anti-linear; it associates to each $v \in \mathcal{H}$ a linear form as usual, $\check{v}=(v, \cdot), \check{v}(u)=(v, u)$. The orthonormality relations of a basis $e_{i}, i \in \mathbb{N}$, then amount to $\check{e}_{j}\left(e_{i}\right)=\delta_{i j}$. If $v_{i}=\check{e}_{i}(v)$ are the components of a vector $v=\sum_{i} v_{i} e_{i}$, then the associated linear form $\check{v}$ has the complex conjugate components, $\check{v}=\sum_{i} v_{i}^{*} \check{e}_{i}$. Suppose that $\mathcal{H}$ carries in addition a representation $\pi$ of some Lie group $G$. Then $\pi(g) v$ has components $(\pi(g) v)_{i}=\sum_{j} \pi(g)_{i j} v_{j}$, where $\pi(g)_{i j}:=\check{e}_{i}\left(\pi(g) e_{j}\right)$ are the matrix elements of $\pi(g)$. Note that in these conventions $\pi(g)$ acts by 'matrix multiplication' on the components $v_{j}=\check{e}_{j}(v)$. We define the conjugate representation $\check{\pi}$ on $\check{\mathcal{H}}$ by $\left.(\check{\pi}(g) \check{v})(u):=\check{v}\left(\pi\left(g^{-1}\right) u\right)\right)$. Since $\left((\check{\pi}(g) \check{v})^{\vee}, u\right)=$ 
$(\check{\pi}(g) \check{v})(u)=\check{v}\left(\pi\left(g^{-1}\right) u\right)=\left(v, \pi\left(g^{-1}\right) u\right)=(\pi(g) v, u)$, this amounts to $[\check{\pi}(g) \check{v}]^{\vee}=\pi(g) v$, or $\check{\pi}(g) \check{v}=[\pi(g) v]^{\vee}$. In particular $\check{\pi}(g) \check{v}$ has components $(\check{\pi}(g) \check{v})_{i}=\sum_{j} \pi(g)_{i j}^{*} v_{j}^{*}$, if $v_{j}$ are the components of $v \in \mathcal{H}$.

For separable Hilbert spaces $\mathcal{H}_{1}$ and $\mathcal{H}_{2}$ with orthonormal bases $e_{i}, i \in \mathbb{N}$, and $f_{j}, j \in \mathbb{N}$, repectively, we define the tensor product $\mathcal{H}_{2} \otimes \check{\mathcal{H}}_{1}$ as the Hilbert space spanned by $f_{i} \check{e}_{j}=: f_{i} \otimes \check{e}_{i}$ and completed with respect to the inner product $\left(f_{i} \otimes \check{e}_{j}, f_{k} \otimes \check{e}_{l}\right)_{2}=\delta_{i k} \delta_{j l}$. The tensor product $\mathcal{H}_{2} \otimes \check{\mathcal{H}}_{1}$ can be canonically identified with the space $\mathcal{J}_{2}\left(\mathcal{H}_{1}, \mathcal{H}_{2}\right)$ of Hilbert-Schmidt operators $F: \mathcal{H}_{1} \rightarrow \mathcal{H}_{2}$, equipped with the inner product $\left(F, F^{\prime}\right)_{2}=$ $\operatorname{Tr}\left[F^{\dagger} F^{\prime}\right]=\sum_{i j} F_{j i}^{*} F_{j i}^{\prime}$, where $F=\sum_{i j} f_{i} F_{i j} \check{e}_{j}$ are the components of $F$. The isometry $\mathcal{H}_{2} \otimes \check{\mathcal{H}}_{1} \rightarrow \mathcal{J}_{2}\left(\mathcal{H}_{1}, \mathcal{H}_{2}\right)$ is simply given by the extension of $\left(\sum_{i} v_{i} f_{i}\right) \otimes\left(\sum_{j} w_{j} e_{j}\right)^{\vee} \mapsto$ $\sum_{i j} v_{i} w_{j}^{*} f_{i} \check{e}_{j}$. (This isometry was in fact already used before in the identification $f_{i} \check{e}_{j}=$ $f_{i} \otimes \check{e}_{i}$.)

Suppose now that $\mathcal{H}_{1}, \mathcal{H}_{2}$ carry unitary representations $\pi_{1}, \pi_{2}$, respectively. Then $\mathcal{H}_{2} \otimes$ $\check{\mathcal{H}}_{1}$ carries a unitary representation $\pi_{2} \times \check{\pi}_{1}$ of $G \times G$, the outer tensor product of $\pi_{2}$ and $\check{\pi}_{1}$. It is given by $\left(\pi_{2} \times \check{\pi}_{1}\right)\left(g_{2}, g_{1}\right)\left(v_{2} \otimes \check{v}_{1}\right):=\pi_{2}\left(g_{2}\right) v_{2} \otimes \check{\pi}_{1}\left(g_{1}\right) \check{v}_{1}$. In the realization as Hilbert-Schmidt operators this means

$$
\left(\pi_{2} \times \check{\pi}_{1}\right)\left(g_{2}, g_{1}\right) F=\pi_{2}\left(g_{2}\right) F \pi_{1}\left(g_{1}\right)^{\dagger}=\pi_{2}\left(g_{2}\right) F \pi_{1}\left(g_{1}\right)^{-1} .
$$

The outer tensor product of two unitary representations is irreducible if and only if both constituents are. The diagonal representation $\left(\pi_{2} \otimes \check{\pi}_{1}\right)(g):=\left(\pi_{2} \times \check{\pi}_{1}\right)(g, g)$ of $G$ is called the inner tensor product of $\pi_{2}$ and $\check{\pi}_{1}$; of course it is in general not irreducible if $\pi_{2}$ and $\check{\pi}_{1}$ are.

4. Relation to amenability: The support of the Plancherel measure in (A.1) also reflects the amenability or nonamenability of the underlying group (see [42] for the definition of amenable topological groups). For a continuous unitary representation $\pi$ of a locally compact group $G$ the support of $\pi$ is the set $\sigma \in \widehat{G}$ weakly contained in $\pi$. Here $\pi_{1}$ is said to be weakly contained in $\pi_{2}$ if every function of positive type can be approximated, uniformly on compact subsets of $G$, by finite sums of functions of positive type associated with $\pi_{2}$. Here functions of positive type can be identified with the diagonal matrix elements $g \mapsto(v, \pi(g) v)$ of a representation. By definition $\widehat{G}_{r}$, the reduced dual of $G$, is the support of the (left or right) regular representation of $G$; see 9], Definitions 18.1.7 and 18.3.1. An amenable locally compact group $G$ is characterized by the property that $\widehat{G}_{r}=\widehat{G}$ (9, Prop. 18.3.6). In fact, whenever $\widehat{G}_{r}$ weakly contains a single finite dimensional continuous unitary representation $\widehat{G}_{r}=\widehat{G}$ follows (므, Prop. 18.3.6 and Addendum 18.9.5). For the group itself one has: a connected semisimple Lie group with a finite center is amenable if and only if it is compact (see [42] or [60], Prop. 4.1.8). All the noncompact (linear reductive) Lie groups considered here are therefore nonamenable. It follows that $\widehat{G}_{r}$ is a proper subset of $\widehat{G}$ and that $\widehat{G}_{r}$ cannot contain any finite dimensional continuous unitary representation. In contrast the Euclidean group $\operatorname{ISO}(N)$ considered in Appendix $\mathrm{C}$ is amenable. 
Sometimes also the concept of an amenable representation is useful, which relates to (A.6). Specifically one is interested in situations where $\pi_{1} \otimes \pi_{2}$ contains the unit representation of $G$, i.e. the singlet. A simple case is when $\pi$ is finite dimensional; then $\pi \otimes \check{\pi}$ always contains the unit representation (as $F=\mathbb{I}$ by (A.6) is clearly an invariant tensor). More generally one has ([5], Prop. A.1.11 and Corollary A.1.12): Let $\pi_{1}, \pi_{2}$ be unitary representations of the Lie group $G$ on $\mathcal{H}_{1}, \mathcal{H}_{2}$. Then the following are equivalent: (i) $\pi_{1} \otimes \pi_{2}$ contains the unit representation. (ii) there exists a finite dimensional representation $\pi$ which is a subrepresentation of both $\pi_{1}$ and $\pi_{2}$. Further, if $\pi_{1}$ is irreducible condition (ii) can be replaced by: (ii)' $\pi_{1}$ is finite dimensional and is contained in $\check{\pi}_{2}$. The unitary representation $\pi$ is called amenable if for $\pi_{1}=\pi, \pi_{2}=\check{\pi}$ any one of the conditions (i)-(iii) is satisfied. This in turn can be shown to be equivalent to [4]: a unitary representation $\pi$ of a Lie group $G$ on a Hilbert space $\mathcal{H}$ is amenable if there exists a positive linear functional $\omega$ over $\mathcal{B}(\mathcal{H})$ (the $C^{*}$-algebra of bounded linear operators on $\mathcal{H}$ ) such that $\omega\left(\pi(g) T \pi(g)^{-1}\right)=\omega(T)$, for all $g \in G$ and all $T \in \mathcal{B}(\mathcal{H})$. Further a locally compact group is amenable iff every $\pi \in \widehat{G}$ is amenable [4. For a simple noncompact Lie group (connected with finite center and rank $>1$ ) the only amenable representations are those containing the trivial one.

5. Characters: The coefficient functions $g \mapsto \pi_{\sigma}(g)_{i j}$ form a unitary irreducible representation, viz

$$
\begin{aligned}
& \pi_{\sigma}\left(g_{1} g_{2}\right)_{i j}=\sum_{k} \pi_{\sigma}\left(g_{1}\right)_{i k} \pi_{\sigma}\left(g_{2}\right)_{k j}, \\
& \pi_{\sigma}(e)_{i j}=\delta_{i j}, \quad \pi_{\sigma}\left(g^{-1}\right)_{i j}=\left[\pi_{\sigma}(g)_{j i}\right]^{*} .
\end{aligned}
$$

For a compact Lie group the coefficients also obey orthogonality and completeness relations essentially summarizing the content of the Plancherel (or Peter-Weyl) expansion. In the case of a noncompact Lie group these have no direct counterpart in that double sums over products of the matrix coefficients or traces diverge. Instead character functions and the associated spectral projectors provide the appropriate substitute for orthogonality and completeness relations.

Characters are defined as follows [55]. Since $\widehat{\phi}(\sigma)$ is a trace class operator for all $\phi \in \mathcal{D}$ the trace

$$
\boldsymbol{\Theta}_{\sigma}(\phi):=\sum_{i} \widehat{\phi}(\sigma)_{i i}=\sum_{i} \int_{G} d g \phi(g) \pi_{\sigma}\left(g^{-1}\right)_{i i},
$$

is well-defined and independent of the choice of orthonormal basis on $\mathcal{L}_{\sigma}$. Thus $\Theta_{\sigma}: \mathcal{D} \rightarrow$ $\mathbb{C}$ is a distribution over $\mathcal{D}$ for every unitary irreducible representation $\pi_{\sigma}$. It characterizes such a representation in that $\boldsymbol{\Theta}_{\sigma_{1}}=\boldsymbol{\Theta}_{\sigma_{2}}$ holds if and only if the representations $\pi_{\sigma_{1}}$ and $\pi_{\sigma_{2}}$ are unitarily equivalent. A representation $\pi_{\sigma}$ is called tempered if the distribution $\Theta_{\sigma}$ extends continously to $\mathcal{S}(G)$, the Schwartz space of functions $\phi \in \mathcal{C}^{\infty}(G)$ such that $\phi$ and all its derivatives are square integrable on $G$. The sum in (A.8) can of course not be pulled inside the integral: as all eigenvalues of $\pi_{\sigma}(g)$ have modulus one the sum over $\pi_{\sigma}(g)_{i i}$ diverges. However for the class of Lie groups considered a regularity theorem 
ensures the existence of a locally integrable function $\Theta_{\sigma}$ such that

$$
\Theta_{\sigma}(\phi)=\int_{G} d g \phi(g) \Theta_{\sigma}\left(g^{-1}\right) \quad \text { for all } \phi \in \mathcal{D}
$$

The function $\Theta_{\sigma}$ is constant on conjugacy classes in the sense that $\Theta_{\sigma}\left(g g^{\prime} g^{-1}\right)=\Theta_{\sigma}\left(g^{\prime}\right)$ for all $g \in G$ and $g^{\prime} \in G^{\prime}$. Here $G^{\prime}$ is a dense open subset of $G$ characterized by the fact that each of its elements lies in precisely one Cartan subgroup $H$ of $G$, see e.g. [55. The character function $\Theta_{\sigma}$ is also an eigenfunction of $Z(G)$, the abelian algebra of all bi-invariant differential operators on $G$. In terms of them the Plancherel expansion (A.2) can be rewritten as

$$
\phi(g)=\int_{\widehat{G}_{r}} d \nu(\sigma)\left(\Theta_{\sigma} * \phi\right)(g), \quad \phi \in \mathcal{D}
$$

Here

$$
\left(\Theta_{\sigma} * \phi\right)(g):=\int_{G} d g^{\prime} \Theta_{\sigma}\left(g g^{\prime-1}\right) \phi\left(g^{\prime}\right)=\sum_{i} \int_{G} d g^{\prime} \phi\left(g^{\prime}\right) \sum_{j} \pi_{\sigma}(g)_{i j} \pi_{\sigma}\left(g^{\prime-1}\right)_{j i}
$$

Due to the properties of $\Theta_{\sigma}$ the above tempered irreducible representations come in families parameterized by conjugacy classes of Cartan subgroups of $G$.

The bi-invariant function $\left(g, g^{\prime}\right) \mapsto \Theta_{\sigma}\left(g g^{\prime-1}\right)$ in (A.11) can be viewed as a 'regularized' version of the formal double sum that would arise by pulling the sum over $i$ inside the integral. There are two natural ways to achieve such a regularization.

One is by performing averages over Borel sets in $\widehat{G}_{r}$, which gives rise to spectral projectors:

$$
\begin{aligned}
& E_{I}\left(g g^{\prime-1}\right):=\int_{I} d \nu(\sigma) \Theta_{\sigma}\left(g g^{\prime-1}\right), \\
& \int d g E_{I}\left(g_{1} g^{-1}\right) E_{J}\left(g g_{2}^{-1}\right)=E_{I \cap J}\left(g_{1} g_{2}^{-1}\right), \\
& E_{\widehat{G}_{r}}\left(g g^{-1}\right)=\int_{\widehat{G_{r}}} d \nu(\sigma) \Theta_{\sigma}\left(g g^{\prime-1}\right)=\delta\left(g, g^{\prime}\right) .
\end{aligned}
$$

6. K-finite functions: A literal way to take the sum over $i$ in (A.11) inside the integral is by restricting the class of functions $\phi$ to the $K$-finite ones. A function $f \in \mathcal{C}_{c}^{\infty}(G)$ is called left (resp. right) $K$-finite ([57] p.236) if the set $\{f(k g), k \in K\}$ (resp. $\{f(g k), k \in K\})$ lies in a finite dimensional subspace of $\mathcal{C}(G)$, the continuous functions on $G$. It is called (bi)-K-finite if both holds. Let $K \ni k \mapsto r_{\kappa}(k), \kappa \in \widehat{K}$, be the unitary irreducible 
representation of $K$ with hightest weight $\kappa$ on a vector space $V_{\kappa}, d_{\kappa}:=\operatorname{dim} V_{\kappa}$. Their characters $k \mapsto \chi_{\kappa}(k):=\operatorname{Tr}\left[r_{\kappa}(k)\right]$ obey $\chi_{\kappa} * \chi_{\kappa^{\prime}}=\delta_{\kappa, \kappa^{\prime}} d_{\kappa}^{-1} \chi_{\kappa}$ (where we took $\kappa$ to label a unitary equivalence class and $*$ denotes the convolution product with respect to $K)$. For any finite subset $I \subset \widehat{K}$ then $E_{I}:=\sum_{\kappa \in I} d_{\kappa} \chi_{\kappa}$ is the spectral projector; in particular $E_{I} * E_{J}=E_{I \cap J}$. The Fourier expansion takes the form $f=\sum_{\kappa \in \widehat{K}} d_{\kappa} f * \chi_{\kappa}$ and converges in the $L^{2}(K)$ norm. One can view the characters as functions on $G$ with support on $K$ only and convolute functions in $\mathcal{C}_{c}^{\infty}(G)$ with the projectors $E_{I}$ (with the convention $\left.(f * h)(g)=\int d g^{\prime} f\left(g g^{\prime-1}\right) h\left(g^{\prime}\right)=\int d g^{\prime} f\left(g^{\prime-1}\right) h\left(g^{\prime} g\right)\right)$. Then ([57], p.237)

$$
f \in \mathcal{C}_{c}^{\infty}(G) \text { is } \begin{cases}\text { left } K \text {-finite } & \text { iff } \quad E_{I} * f=f, \\ \text { right } K \text {-finite } & \text { iff } \quad f * E_{I}=f,\end{cases}
$$

for some finite $I \subset \widehat{K}$. The function $f$ is bi-K-finite if $E_{I} * f * E_{I}=f$ holds.

A representation $\pi$ of $G$ on a Hilbert space $\mathcal{H}$ is called $K$-finite ([57], p.232) if its restriction to the compact subgroup $K$ is unitary and decomposes into a unitary sum of irreducibles $\left(r_{\kappa}, V_{\kappa}\right), \kappa \in \widehat{K}$, each occuring with finite multiplicity $m_{\kappa}$. That is, $\mathcal{H}=\bigoplus_{\kappa \in \widehat{K}} m_{\kappa} V_{\kappa}$ as a representation of $K$. Let $P_{\kappa}: \mathcal{H} \rightarrow m_{\kappa} V_{\kappa}$ be the orthogonal projection. Note that an alternative characterization of a $K$-finite representation $\pi$ is that $P_{\kappa}$ is an operator of finite rank on $\mathcal{H}$ for all $\kappa \in \widehat{K}$. A vector $v \in \mathcal{H}$ is called $K$-finite if $\pi(k) v, k \in K$, generates a finite dimensional subspace of $\mathcal{H}([29]$, p.25). Evidently this is the case iff $P_{I} v=v$ for some finite $I \subset \widehat{K}$. To study the relation between $K$ finite functions and $K$-finite vectors the following explicit realization of the projectors is useful:

$$
P_{\kappa} v=d_{\kappa} \int_{K} d k \chi_{\kappa}\left(k^{-1}\right) \pi(k) v, \quad v \in \mathcal{H} .
$$

Consistency requires that $\pi(k) \circ P_{\kappa}=P_{\kappa} \circ \pi(k), k \in K$, which indeed is a property of the right hand side of (A.14) using that the character $\chi_{\kappa}$ is constant on $K$ conjugacy classes. To verify A.14 first note that the matrix elements of $\pi(k), k \in K$, are blockdiagonal in the basis $\left\{e_{\kappa, s}, s=0, \ldots, m_{\kappa} d_{\kappa}-1, \kappa \in \widehat{K}\right\}$, where $e_{\kappa, m+d_{\kappa}}=e_{\kappa, m}, m=0, \ldots, d_{\kappa}-1$, is an orthonormal basis of $V_{\kappa}$. Explicitly

$$
\left(e_{\kappa^{\prime} s^{\prime}}, \pi(k) e_{\kappa s}\right)=\delta_{\kappa^{\prime}, \kappa} r_{\kappa}(k)_{s^{\prime} s} .
$$

Any $v \in \mathcal{H}$ can by assumption be expanded as $v=\sum_{\kappa, s}\left(e_{\kappa s}, v\right) e_{\kappa s}$ and $\pi(k)$ acts on $m_{\kappa} V_{\kappa}$ as the blockdiagonal matrix $r_{\kappa}(k)$. Thus, to verify (A.14 one only has to show that $\left(e_{\kappa^{\prime} m^{\prime}}, P_{\kappa} v\right)=\delta_{\kappa^{\prime}, \kappa}\left(e_{\kappa m^{\prime}}, v\right)$. Taking the trace over $m_{1}=m_{2}$ in

$$
\int_{K} d k r_{\kappa}\left(k^{-1}\right)_{m_{1} m_{2}} r_{\kappa^{\prime}}(k)_{m_{3} m_{4}}=\frac{1}{d_{\kappa}} \delta_{\kappa \kappa^{\prime}} \delta_{m_{1} m_{4}} \delta_{m_{2} m_{3}}
$$

(see e.g. 3], p.170) this readily follows. 
The interest of these constructions lies in fact that any $K$-finite representation is a direct orthogonal sum of unitary irreducible representations ([57], Lemma 8.6.22). The irreducible representions are of course also $K$ finite and for them explicit bounds on the multiplicities are available. For the purposes here two results are relevant: Let $G$ be a semi-simple linear connected real Lie group and $K$ a maximal compact subgroup. Then $\pi_{\sigma}$ is $K$-finite for any $\sigma \in \widehat{G}$ and the irreducible representation $\kappa \in \widehat{K}$ occurs in $\left.\pi_{\sigma}\right|_{K}$ at most with multiplicity $d_{\kappa}$ (see [9], p.331). A theorem by Harish-Chandra (58], p.319) states that essentially the same is true for any connected semi-simple Lie group with finite center (with a technically slightly different notion of irreducibility). On account of these multiplicity bounds one can identify $\mathcal{L}_{\sigma}$ as a vector space with $\bigoplus_{\kappa} m_{\kappa} V_{\kappa}$ and since the multiplicities $m_{\kappa} \leq d_{\kappa}$ do not exceed those in the decomposition of $L^{2}(K)$ (which equal $d_{\kappa}$ ) one can identify each $\mathcal{L}_{\sigma}$ with a subspace of $L^{2}(K)$. In certain cases the upper bound on the multiplicities is even sharper. In the case $G=\mathrm{SO}_{0}(1, N), K=\mathrm{SO}(N)$ we focus on, each $\kappa \in \widehat{K}$ can occur at most once in $\left.\pi_{\sigma}\right|_{K}$, by a result due to Dixmier [10]. Another case when this happens is for $G=\mathrm{SL}(2, \mathbb{C})$ and $K=\mathrm{SU}(2)$, see [58], p.317, where also the general conditions for $m_{\kappa} \leq 1$ are discussed.

Let now $\pi$ be a $K$-finite representation and write $\pi(\psi)=\int d g \psi(g) \pi(g)$ for $\psi \in \mathcal{C}_{c}^{\infty}(G)$. Using Eq. (A.14) one readily verifies the following relations

$$
\pi\left(E_{\kappa} * \psi\right)=\pi(\psi) P_{\kappa} \quad \pi\left(\psi * E_{\kappa}\right)=P_{\kappa} \pi(\psi)
$$

and similarly for the two-sided projections. The same holds for the Fourier coefficients $\int d g \psi(g) \pi\left(g^{-1}\right)=(\pi * \psi)(e)$. For an irreducible representation $\pi_{\sigma}, \sigma \in \widehat{G}$, and $\psi \in L^{1}(G) \cap L^{2}(G)$, the relations (A.17) then imply the following decomposition of the Hilbert-Schmidt operator $\widehat{\psi}(\sigma): \mathcal{L}_{\sigma} \rightarrow \mathcal{L}_{\sigma}$ :

$$
\begin{aligned}
\widehat{\psi}(\sigma) & =\sum_{\kappa_{2}, s_{2}, \kappa_{1} s_{1}} e_{\kappa_{2} s_{2}} \widehat{\psi}(\sigma)_{\kappa_{2} s_{2}, \kappa_{1} s_{1}} \check{e}_{\kappa_{1} s_{1}} \\
& =\sum_{\kappa_{2}, \kappa_{1}} P_{\kappa_{2}} \widehat{\psi}(\sigma) P_{\kappa_{1}}=\sum_{\kappa_{2}, \kappa_{1}}\left(E_{\kappa_{1}} * \psi * E_{\kappa_{2}}\right) \widehat{-}(\sigma) .
\end{aligned}
$$

For left $K$-finite functions the sum over $\kappa_{1}$ is finite, for right $K$-finite functions the one over $\kappa_{2}$ is, and for bi- $K$ finite functions both sums are finite. Here $\check{e}_{\kappa s}$ is the basis dual to $e_{\kappa s}, s=0, \ldots, m_{\kappa} \operatorname{dim} V_{\kappa}-1$. In particular $\widehat{\psi}(\sigma)_{\kappa_{2} s_{2}, \kappa_{1} s_{1}}=\check{e}_{\kappa_{2} s_{2}}\left(\widehat{\psi}(\sigma) e_{\kappa_{1} s_{1}}\right)=$ $\left(e_{\kappa_{2} s_{2}}, \widehat{\psi}(\sigma) e_{\kappa_{1} s_{1}}\right)_{\sigma}$.

7. Harmonic analysis on $G / K$ : If instead of Fourier analyzing functions in $L^{2}(G)$ one is only interested in $L^{2}(G / K)$ functions, where $Q \simeq G / K$ is a symmetric space of noncompact type, the harmonic analysis simplifies considerably. In group theoretical terms it amounts to the decomposition of the quasiregular representation $\ell_{1}$ of $G$ on $L^{2}(Q)$. We resume the specifications and notations of Section A.1; in particular $K$ is a maximal compact subgroup of $G$, so that $Q$ is an indecomposable Riemannian symmetric space. The key simplification is that for the harmonic analysis on $G / K$ only a subset 
of the principal series representations proper is needed (see 23] and [24, Section IV.7). Recall that the principal series representations proper are those induced by the minimal parabolic subgroup $P=N A M$, where $G=N A K$ is the Iwasawa decomposition of $G$ and $M$ is the centralizer of $A$ in $K$. The inducing construction will be described below. The upshot is that the principal series representations $\pi_{\nu, \xi}$ are labeled by a character $\nu: A \rightarrow U(1)$ of $A$ and by a unitary (finite dimensional) irreducible representation $\xi \in \widehat{M}$ of $M$. The principal series representations associated with the singlet $\xi=0$ of $M$ are called the spherical principal series representations (or minimal or class 1 principal series; see e.g. [58], Vol.1, p.462). These representations $\pi_{\nu, 0}$ are thus labeled by elements of $\widehat{A}$ only, which can be identified with a subset of $\mathbb{R}^{\operatorname{dim} A}$. We write $\widehat{Q}$ for this subset and label the characters $\nu=\nu_{\omega}$ and the representations $\pi_{\omega, 0}:=\pi_{\nu_{\omega}, 0}$ by points in $\omega \in \widehat{Q}$. The abstract definition of $\widehat{G / K}$ as the subset of $\widehat{G}_{r}$ needed for the harmonic analysis on $G / K$ thus turns into the bijection $\widehat{G / K} \simeq \widehat{Q} \subset \mathbb{R}^{\operatorname{dim} A}$. Moreover the Plancherel measure $\left.d \nu(\sigma)\right|_{\widehat{G / K}}$ restricted to the spherical principal series is absolutely continuous with respect to the Lebesgue measure $d \omega$ on $\mathbb{R}^{\operatorname{dim} A}$,

$$
\left.d \nu(\sigma)\right|_{\widehat{G / K}}=\frac{d \omega}{|c(\omega)|^{2}}
$$

Here $c(\omega)$ is the Harish-Chandra $c$-function, for which an explicit formula in terms of the structure of $G / K$ is known. Concerning the $K$ content of the $\pi_{\omega, 0}$ representations, it is known that all of them contain the $K$-singlet with multiplicity 1 ([23], p.414). As a consequence the representations $\pi_{\omega, 0}$ can be set into one-to-one correspondence to $K$ spherical functions. A continuous function $p$ on $G$ is called $K$-spherical ([24], p.357) if it satisfies

$$
\int d k p\left(g_{1} k g_{2}\right)=p\left(g_{1}\right) p\left(g_{2}\right)
$$

This implies that $p$ is $K$-bi-invariant and normalized $p(e)=1$.

One can explicate this structure by writing out the coefficients of $\pi_{\omega, 0}$ in the $K$-adapted basis of Section 2.4 using (A.15). An equivalent characterization of the matrix elements in (A.15) is then

$$
\pi_{\omega, 0}\left(k_{1} g k_{2}\right)_{\kappa s, \kappa^{\prime} s^{\prime}}=\sum_{s_{1}, s_{2}} r_{\kappa}\left(k_{1}\right)_{s s_{1}} \pi_{\omega, 0}(g)_{\kappa s_{1}, \kappa^{\prime} s_{2}} r_{\kappa}\left(k_{2}\right)_{s_{2} s^{\prime}}
$$

In particular one sees: $\pi_{\omega, 0}(g)_{00, \kappa s}$ is left $K$-invariant, $\pi_{\omega, 0}(g)_{\kappa s, 00}$ is right $K$-invariant, and $\pi_{\omega, 0}(g)_{00,00}$ is $K$-spherical. The right K-invariant functions $g \mapsto\left[\pi_{\omega, 0}(g)\right]_{\kappa s, 00}$ are sufficient for the harmonic analysis on the right coset space $G / K$. To see this observe that for a left $K$-invariant function $(\phi(g k)=\phi(g)$, for all $k \in K)$ the decomposition 
(A.2) specializes to

$$
\begin{aligned}
\widehat{\phi}(\sigma)_{\kappa s, \kappa^{\prime} s^{\prime}} & =\int_{G / K} d \gamma_{G}(g K) \phi(g K) \int_{K} d \gamma_{K}(k) \pi_{\sigma}\left(k^{-1} g^{-1}\right)_{\kappa s, \kappa^{\prime} s^{\prime}}=\widehat{\phi}(\sigma)_{00, \kappa s} \delta_{\kappa^{\prime}, 0} \delta_{s^{\prime}, 0}, \\
\phi(g K) & =\int_{\widehat{G / K}} d \nu(\sigma) \sum_{\kappa, s} \widehat{\phi}(\sigma)_{00, \kappa s} \pi_{\sigma}(g K)_{\kappa s, 00} .
\end{aligned}
$$

Here we wrote $\widehat{G / K}$ for the subset of representations in $\widehat{G}_{r}$ for which the matrix elements $\widehat{\phi}(\sigma)_{\kappa s, 00}$ are nonzero. According to the above results it consists of spherical principal series representations only, and there is a bijection $\widehat{G / K} \simeq \widehat{Q} \subset \mathbb{R}^{\operatorname{dim} A}$ to a subset of $\mathbb{R}^{\operatorname{dim} A}$. Combined with (A.19) this allows for a very explicit description of the harmonic analysis on $G / K$. Via the Iwasawa decomposition the section $g_{s}(q)$ provides a one-to-one correspondence between points $q \in Q$ and right $K$-orbits (recall that $g_{s}(q)$ equals $n a$ viewed as a function of $q$ ). We define

$$
E_{\omega, \kappa s}(q):=\left[\pi_{\omega, 0}\left(g_{s}(q)\right)\right]_{00, \kappa s}
$$

The functions $E_{\omega, \kappa s}(q)$ are equivariant with respect to $\pi_{\omega, 0}$, i.e.

$$
E_{\omega, \kappa s}\left(g^{-1} q\right)=\sum_{\kappa^{\prime} s^{\prime}} E_{\omega, \kappa^{\prime} s^{\prime}}(q) \pi_{\omega, 0}(g)_{\kappa^{\prime} s^{\prime}, \kappa s} .
$$

The spectral synthesis formulas then assume the form

$$
\begin{aligned}
\psi(q) & =\int_{\widehat{Q}} \frac{d \omega}{|c(\omega)|^{2}} \sum_{\kappa s} \widehat{\psi}(\omega)_{\kappa s} E_{\omega, \kappa s}(q), \\
\widehat{\psi}(\omega)_{\kappa s} & =\int_{Q} d \gamma_{Q}(q) \psi(q) E_{\omega, \kappa s}(q) .
\end{aligned}
$$

Finally we mention that in the fiber decomposition

$$
L^{2}(G / K) \simeq \int^{\oplus} \frac{d \omega}{|c(\omega)|^{2}} \mathcal{L}_{\omega}, \quad \ell_{1} \simeq \int^{\oplus} \frac{d \omega}{|c(\omega)|^{2}} \pi_{\omega, 0}
$$

all fiber spaces $\mathcal{L}_{\omega}$ are isometric to $L^{2}(K / M)$. This can be understood from the fact that generic (nonspherical) principal series representations can be modeled on $L^{2}(K)$ (see below); so for the $M$ singlets this gives model spaces isometric to $L^{2}(K / M)$.

8. Principal series: As is clear from the preceeding discussion the principal series of unitary irreducible representations is at the core of the harmonic analysis for noncompact Lie groups. For complex (noncompact semisimple connected) Lie groups (with a finite center) it suffices in fact for the harmonic analysis. Since we will need a number of results 
that occur in its construction we present here a concise but in principle selfcontained summary thereof. For definiteness we consider again the class of linear reductive Lie groups, though everything goes through also for arbitary non-compact semi-simple Lie groups with a finite center.

The principal series arise as special cases of so-called multiplier representations defined as follows. Consider a diffentiable manifold $\mathcal{M}$ carrying a differentiable $G$ action $\mathcal{M} \ni$ $m \mapsto g . m \in \mathcal{M}$. For a finite dimensional vector space with inner product $\langle,\rangle_{V}$ let $L^{2}(\mathcal{M}, V)$ be the Hilbert space of functions $f: \mathcal{M} \rightarrow V$ square integrable with respect to

$$
\begin{aligned}
& \left(f_{1}, f_{2}\right):=\int d \gamma(m)\left\langle f_{1}(m), f_{2}(m)\right\rangle_{V} \\
& \left\langle f_{1}(m), f_{2}(m)\right\rangle_{V}:=\sum_{s} f_{1}(m)_{s}^{*} f_{2}(m)_{s} .
\end{aligned}
$$

Let further $G \times \mathcal{M} \ni(g, m) \mapsto \chi(g, m), \chi(g, m): V \rightarrow V$, be a cocycle satisfying

$$
\chi\left(g_{1} g_{2}, m\right)=\chi\left(g_{1}, m\right) \chi\left(g_{2}, g_{1} \cdot m\right), \quad \chi(e, m)=\mathbb{I} .
$$

Set

$$
[\pi(g) f](m):=\chi(g, m) f(g . m) \sqrt{\frac{d \gamma_{g}(m)}{d \gamma}},
$$

where $d \gamma_{g}=d(\gamma \circ g)$ is the translated measure and $d \gamma_{g} / d \gamma$ is the Radon-Nikodym derivative. The latter ensures both the representation property and the unitarity with respect to the inner product (A.27)

$$
\pi\left(g_{1}\right) \pi\left(g_{2}\right)=\pi\left(g_{1} g_{2}\right), \quad\left(\pi\left(g_{1}\right) f_{1}, \pi\left(g_{2}\right) f_{2}\right)=\left(f_{1}, f_{2}\right)
$$

One now applies this construction principle to the group manifold $K$, where $K$ is the maximal compact subgroup of $G$. Via the Iwasawa decomposition $G=N A K=B K$ it carries a $G$-action induced by the right translations. Explicitly let $g=n(g) a(g) k(g)=$ $b(g) k(g)$ be the unique Iwasawa decomposition of some $g \in G$. Then

$$
B \backslash G \simeq K, \quad k_{0} g=b\left(k_{0} g\right) k\left(k_{0} g\right), \quad k_{0} \in K,
$$

that is, the coset space $B \backslash G$ of left equivalence classes $b g \sim g, b \in B$, is isometrically identified with $K$ by picking the representative $k\left(k_{0} g\right)=: g\left[k_{0}\right]$ in the Iwasawa decomposition. For each $g \in G$ the map $k_{0} \mapsto g\left[k_{0}\right]$ defines a diffeomorphism on $K$, which is the $G$-action on $K$ needed to define the multiplier representation (A.29). We write $d(g[k])$ for the translated bi-invariant Haar measure $d k$ in $K$ and $d(g[k]) / d k$ for the corresponding Radon-Nikodym derivative. According to the general construction

$$
[\pi(g) f](k)=\chi(g, k) f(g[k]) \sqrt{\frac{d(g[k])}{d k}},
$$


defines a unitary representation on $L^{2}(K, V)$ for any cocycle $\chi(g, k): V \rightarrow V$. It remains to compute the Radon-Nikodym derivative. It comes out to be $\Delta(b(k g))^{-1}$, where $b \mapsto \Delta(b)$ is the (right) modular function of the (non-unimodular) subgroup $B=A N$. That is,

$$
\int_{K} d k f(k)=\int_{K} d k f(g[k]) \Delta(b(k g))^{-1},
$$

for all $f \in \mathcal{C}_{c}(K)$. The proof is simple and instructive, so we present it here, also in order to set the conventions (see e.g. [29], p.44f and [55], p.83f), with the opposite conventions). Let $d b$ be the left invariant Haar measure on $B$ and $d g, d k$ the bi-invariant Haar measures on $G, K$, respectively. Then

$$
d g=d b d k \text { for functions of } g=b k,
$$

where the order $b k$ (rather than $k b$ ) is important. Indeed, by definition the map $B \times$ $K \rightarrow G,(b, k) \mapsto b k$ is an isomorphism. There exists therefore an analytical function $J: B \times K \rightarrow \mathbb{R}^{+}$such that $d g=J(b, k) d b d k$. Since $d g=d\left(b_{0} g k_{0}\right)$ for all $b_{0} \in B, k_{0} \in K$ it follows that $J\left(b_{0} b, k k_{0}\right)=J(b, k)$. Thus $J$ must be a constant, which by a change of normalization can be set to unity. Note that if functions of $k b$ were considered the counterpart of (A.34) would read $d g=d_{r} b d k$, with $d_{r} b$ the right Haar measure on $B$. Our convention for the modular function is that of [15], p.46ff]

$$
d\left(b b_{0}\right)=\Delta\left(b_{0}\right) d b
$$

so that $d_{r} b=\Delta\left(b^{-1}\right) d b$. With these preparations at hand the verification of (A.33) is straightforward. Let $E \in \mathcal{C}_{b}(B)$ be a function such that $\int_{B} d b E(b)=1$. Given an arbitrary $f \in \mathcal{C}_{c}(K)$ define a function $F \in \mathcal{C}_{c}(G)$ by $g=b k \mapsto E(b) f(k)$. Then

$$
\int d k f(k)=\int_{G} d g F(g)=\int_{G} d g F\left(g g_{0}\right)=\int d k d b F\left(b k g_{0}\right)
$$

for all $g_{0} \in G$. By (A.31) $k g_{0}$ decomposes as $k g_{0}=b\left(k g_{0}\right) g_{0}[k]$, so that $F\left(b k g_{0}\right)=$ $E\left(b b\left(k g_{0}\right)\right) f\left(g_{0}[k]\right)$. Inserting into (A.36) and shifting the integration variable $b \rightarrow$ $b b\left(k g_{0}\right)^{-1}$ gives (A.33).

To proceed we describe the action of $G$ on itself in terms of the Iwasawa decomposition $G=N A K$. Converting the relations in [55], p.84 into the present conventions one has

$$
\begin{aligned}
k\left(g_{1} g_{2}\right) & =k\left(k\left(g_{1}\right) g_{2}\right)=g_{2}\left[k\left(g_{1}\right)\right], \\
a\left(g_{1} g_{2}\right) & =a\left(g_{1}\right) a\left(k\left(g_{1}\right) g_{2}\right), \\
n\left(g_{1} g_{2}\right) & =n\left(g_{1}\right)\left[a\left(g_{1}\right) n\left(k\left(g_{1}\right) g_{2}\right) a\left(g_{1}\right)^{-1}\right] .
\end{aligned}
$$


As a check note $n\left(g_{1} g_{2}\right) a\left(g_{1} g_{2}\right) k\left(g_{1} g_{2}\right)=n\left(g_{1}\right) a\left(g_{1}\right)(n a k)\left(k\left(g_{1}\right) g_{2}\right)=g_{1} g_{2}$. In particular it follows that $a(g, k):=a(k g)$ is an $A$-valued cocycle,

$$
a\left(g_{1} g_{2}, k\right)=a\left(g_{1}, k\right) a\left(g_{2}, g_{1}[k]\right), \quad a(e, k)=e .
$$

We evaluate it on two types of continuous group homomorphisms. The first, $\delta: A \rightarrow \mathbb{R}_{+}$, is obtained from

$$
d\left(a n a^{-1}\right)=: \delta(a)^{2} d n, \quad \Delta(a n)=\delta(a)^{2},
$$

where $g=n a k$ is the Iwasawa decomposition. The first relation in (A.39) defines $\delta$, the second readily follows from $d b=d a d n$ for functions of $b=$ an (see [55], p.80, 29], p.39). The second group homomorphism $\nu: A \rightarrow U(1)$ assigns to each $a \in A$ a complex phase $\nu(a) \in U(1)$.

We define a representation of $G$ on $L^{2}(K, V)$ by

$$
\left[\pi_{\nu}(g) f\right](k):=\delta(a(g, k))^{-1} \nu(a(g, k)) f(g[k]) .
$$

Since it is obtained by specialization of (A.32) it is unitary. The following property of $\pi_{\nu}$ will allow one to restrict it to subspaces of $L^{2}(K, V)$ such that the resulting representations are equivalent to the principal series representations sought after. Namely:

(i) The restriction of $\pi_{\nu}$ to $K$ is the right regular representation of $K$, i.e. $\pi_{\nu}\left(k_{0}\right) f(k)=$ $f\left(k k_{0}\right)$. (ii) $\pi_{\nu}$ commutes with the left regular representation of $M$

$$
\ell(m) \circ \pi_{\nu}(g)=\pi_{\nu}(g) \circ \ell(m), \quad \text { for all } m \in M
$$

Here $M$ is the centralizer of $A$ in $K$, that is the subgroup $M \subset K$ whose elements commute with all elements of $A$. Property (i) follows from $a\left(k_{0}, k\right)=e$ and $k_{0}[k]=k k_{0}$. Equation (A.40) is equivalent to

$$
a(m g)=a(g), \quad k(m g)=m k(g), \quad \text { for all } m \in M .
$$

To verify (A.41) it suffices to note that $n(m g)=m n(g) m^{-1}$ since $M$ normalizes $N$. Thus $m g=n(m g) a(m g) k(m g)=m n(g) a(m g) m^{-1} k(m g)$, which gives (A.41).

Let now $m \mapsto r_{\xi}(m), \xi \in \widehat{M}$, be an irreducible representation of $M$ on $V_{\xi}$ and consider

$$
L_{\xi}^{2}(K)=\left\{f \in L^{2}\left(K, V_{\xi}\right) \mid f(m k)=r_{\xi}(m) f(k), m \in M\right\} .
$$

Eq. (A.40) implies that for $f \in L_{\xi}^{2}(K)$ one has $\left[\pi_{\nu}(g) f\right](m k)=r_{\xi}(m)\left[\pi_{\nu}(g) f\right](k)$. Thus $\pi_{\nu}: L_{\xi}^{2}(K) \rightarrow L_{\xi}^{2}(K)$ and the restriction

$$
\pi_{\nu, \xi}(g):=\left.\pi_{\nu}(g)\right|_{L_{\xi}^{2}(K)},
$$


is well defined. The subgroup $P:=N A M$ is a (minimal) parabolic subgroup of $G$ and the representations (A.44) are the $P$-principal series representations of $G$ in the so-called 'compact model'. Explicitly

$$
\left[\pi_{\nu, \xi}(g) f\right](k)=\delta(a(k g))^{-1} \nu(a(k g)) f(g[k]), \quad g=n a k
$$

The standard definition of the $P$-principal series is by 'parabolic induction'. For completeness we briefly recap this construction and verify that it is equivalent to (A.45). First note that in the above notation $\chi_{\nu, \xi}(\mathrm{nam})=\nu(a) r_{\xi}(\mathrm{m})$ is a unitary representation of $P=N A M$ on $V_{\xi}$. Indeed, rearranging the Iwasawa components of $p_{1}, p_{2} \in P$ one finds $p_{1} p_{2}=n\left(p_{1}\right) n\left(m\left(p_{1}\right) a\left(p_{1}\right) p_{2}\right) a\left(p_{1}\right) a\left(p_{2}\right) m\left(p_{1}\right) m\left(p_{2}\right)$, using $a n(g) a^{-1}=n(a g)$ and $m n(g) m^{-1}=n(m g)$. The $P$-principal series is then defined as the representation of $G$ induced by $\chi_{\nu, \xi}, \operatorname{Ind}_{P}^{G} \chi_{\nu, \xi}$. This means one considers the linear space of functions $F: G \rightarrow V_{\xi}$ such that

$$
\begin{aligned}
& F(p g)=\Delta(p)^{-1 / 2} \chi_{\nu, \xi}(p) F(g), \\
& \left(F_{1}, F_{2}\right):=\int_{K} d k\left\langle F_{1}(k), F_{2}(k)\right\rangle_{V_{\xi}},
\end{aligned}
$$

which upon completion with respect to the norm given forms a Hilbert space $\mathcal{H}_{\chi_{\nu, \xi}}$. Here $\Delta$ is the modular function of $P=N A M$, which (since $M$ is compact) coincides with the modular function of $B=N A$ and hence is given by $\Delta($ nam $)=\delta(a)^{2}$. Explicitly the covariance equation in (A.46) thus reads

$$
F(n a m g)=\delta(a)^{-1} \nu(a) r_{\xi}(m) F(g)
$$

The induced representation then is defined as the restriction of the right regular representation to $\mathcal{H}_{\chi_{\nu, \xi}}$,

$$
\left[\operatorname{Ind}_{P}^{G}\left(g_{0}\right) F\right](g):=F\left(g g_{0}\right), \quad F \in \mathcal{H}_{\chi_{\nu, \xi}} .
$$

The unitarity follows from (A.33) applied to $f(k)=\left\langle F_{1}\left(g_{0}[k]\right), F_{2}\left(g_{0}[k]\right)\right\rangle_{V_{\xi}}$. The equivalence to the 'compact model' (A.44), A.45) comes about as follows. Via

$$
F(n a k)=\delta(a)^{-1} \nu(a) f(k)
$$

one can set up a correspondence between functions $F \in \mathcal{C}_{c}(G)$ and $f \in \mathcal{C}_{c}(K)$. Moreover $F \in \mathcal{H}_{\chi_{\nu, \xi}}$ if and only if $f \in L_{\nu, \xi}^{2}(K)$. To see this one rewrites the argument namg in (A.47) as $n a m g=n n(a m g) a a(g) m k(g)$; in this form the equivariance properties are directly mapped onto each other. The square integrability in both function spaces is the same, as $\left.F\right|_{K}=f$ in (A.49). Finally the correspondence (A.49) maps the representations onto each other: We use the relation $k g_{0}=(n a)\left(k g_{0}\right) g_{0}[k]$ to rewrite $g g_{0}=n a k g_{0}$ as

$$
g g_{0}=n n\left(a k g_{0}\right) a a\left(k g_{0}\right) g_{0}[k] \text {. }
$$


From here one easily verifies $\left[\operatorname{Ind}_{P}^{G}\left(g_{0}\right) F\right](g)=F\left(g g_{0}\right)=\left[\pi_{\nu, \xi}\left(g_{0}\right) f\right](k)$, for $g=n a k$. Taken together, the restriction map $\left.F \rightarrow F\right|_{K}$ intertwines the unitary representations $\operatorname{Ind}_{P}^{G} \chi_{\nu, \xi}$ on $\mathcal{H}_{\chi_{\nu, \xi}}$ and $\pi_{\nu, \xi}$ on $L_{\xi}^{2}(K)$.

In summary, the principal series representations of a semisimple Lie group are parameterised by a unitary character $\nu: A \rightarrow U(1)$, i.e. $\nu \in \widehat{A}$, and by an element $\xi \in \widehat{M}$, where $M$ is the centralizer of $A$ in $K$ and $G=N A K$ is the Iwasawa decomposition. From the viewpoint of the Plancherel measure (A.4) they account for the conjugacy classes of Cartan subgroups of the form $H=T \times R$, where the noncompact part $R$ is isomorphic to $\widehat{A}$ (see App.A.1.2), while the compact part $T$ comes from the Cartan subgroup $H_{M}$ of $M$. Schematically $H=\widehat{A} \times H_{M}, \sigma=(\nu, \xi)$. The construction does not ensure irreducibility in itself; typically however principal series representations are irreducible. By Kostant's theorem ( $[58$, Thm. 5.5.2.3) this is the case whenever $G$ is a semisimple connected Lie group with finite center and $P$ is a minimal parabolic subgroup. In particular this holds for all $\mathrm{SO}_{0}(1, N), N \leq 3$, and for the two-fold simply connected covering $\operatorname{Spin}(1, N)$ of $\mathrm{SO}_{0}(1, N)$, with $N$ odd. In rare cases a principal series representation may fail to be irreducible; however it then decomposes into a direct sum of irreducible representations, the number of which cannot exceed the order of the Weyl group (see [58, Vol.1, Corr. 5.5.2.2, p.461).

The action of $\pi_{\nu, \xi}$ on $L_{\xi}^{2}(K)$ and its matrix elements can be described fairly explicitly. To this end set $\pi_{\nu}(\phi):=\int_{G} d g \phi(g) \pi_{\nu}(g)$, for $\phi \in \mathcal{C}_{c}(G)$. Then

$$
\begin{aligned}
{\left[\pi_{\nu}(\phi) f\right]\left(k_{0}\right) } & =\int d k \pi_{\nu}(\phi)\left(k_{0}, k\right) f(k), \\
\pi_{\nu}(\phi)\left(k_{0}, k\right) & =\int d(n a) \phi\left(k_{0}^{-1} n a k\right)\left(\delta^{-1} \nu\right)(a),
\end{aligned}
$$

that is, $\pi_{\nu}(\phi)$ acts as an integral operator with the given kernel. If the function $\phi$ has support only on $K$ the kernel reduces to $\pi_{\nu}(\phi)\left(k_{0}, k\right)=\phi\left(k_{0}^{-1} k\right)$. Both statements follow directly from (A.45) taking into account that $g\left[k_{0}\right]=k\left(k_{0} g\right)$ and $d g=d(n a) d k$ for functions of $g=n a k$.

Since $\pi_{\nu}$ commutes with the left regular representation of $M$, see Eq. (A.40), the kernel obeys

$$
\pi_{\nu}(\phi)\left(m k_{0}, m k\right)=\pi_{\nu}(\phi)\left(k_{0}, k\right), \quad m \in M .
$$

Using the fact that for any $m \in M$ the map $n \mapsto m^{-1} n m$ is a diffeomorphism of $N$ onto itself with unit Jacobian, $d n=d\left(m^{-1} n m\right)$, the invariance (A.52) is also readily verified directly. Both the trace $\operatorname{Tr}\left[\pi_{\nu}(\phi)\right]=\int_{K} d k \pi_{\nu}(\phi)(k, k)$ and the Hilbert-Schmidt norm $\left\|\pi_{\nu}(\phi)\right\|_{2}^{2}=\operatorname{Tr}\left[\pi_{\nu}(\phi)^{*} \pi_{\nu}(\phi)\right]$ are finite for $\phi \in \mathcal{C}_{c}^{\infty}(G)$. This remains true upon restriction to $L_{\xi}^{2}(K)$ where $\pi_{\nu \xi}(\phi)$ basically gives the Fourier coefficients entering the Plancherel decomposition. In detail let

$$
\left[P_{\xi} f\right](k):=\int_{M} d m r_{\xi}\left(m^{-1}\right) f(m k), \quad\left[P_{\xi} f\right](m k)=r_{\xi}(m)\left[P_{\xi} f\right](k)
$$


be the projector from $L^{2}\left(K, V_{\xi}\right)$ to $L_{\xi}^{2}(K)$. Then $\pi_{\nu \xi}(\phi):=\pi_{\nu}(\phi) P_{\xi}=P_{\xi} \pi_{\nu}(\phi)=\widehat{\phi}(\nu, \xi)^{\dagger}$, acts as an integral operator with matrix valued kernel

$$
\begin{aligned}
\pi_{\nu \xi}(\phi)\left(k_{0}, k\right) & =\int_{M} d m \pi_{\nu}(\phi)\left(k_{0}, m k\right) r_{\xi}(m) \\
& =\int_{P} d p \phi\left(k_{0}^{-1} p k\right)\left(\delta^{-1} \nu\right)(p) r_{\xi}(m(p)),
\end{aligned}
$$

where $P=N A M, d p=d(n a) d m$ and $m\left(n^{\prime} a^{\prime} m^{\prime}\right)=m^{\prime}$. Note that the $\ell(M)$ invariance (A.52) of the kernel $\pi_{\nu}(\phi)\left(k_{0}, k\right)$ has turned into a covariance

$$
\pi_{\nu \xi}(\phi)\left(m k_{0}, m k\right)=r_{\xi}(m) \pi_{\nu \xi}(\phi)\left(k_{0}, k\right) r_{\xi}(m)^{-1} .
$$

As noted before, for $\phi \in \mathcal{C}_{c}^{\infty}(G)$ the kernel A.54) defines a trace class operator on $L_{\xi}^{2}(K)$. Both the left and the right action of $\pi_{\nu \xi}(g)$ on $\pi_{\nu, \xi}(\phi)$ produces again a trace class operator whose kernel is readily worked out. One finds

$$
\begin{aligned}
& \text { kernel of } \pi_{\nu, \xi}(g) \pi_{\nu \xi}(\phi)=\pi_{\nu, \xi}(\ell(g) \phi)\left(k, k^{\prime}\right) \\
& =\left(\delta^{-1} \nu\right)(a(k g)) \pi_{\nu, \xi}(\phi)\left(g[k], k^{\prime}\right), \\
& \text { kernel of } \pi_{\nu, \xi}(\phi) \pi_{\nu \xi}(g)=\pi_{\nu, \xi}\left(\rho\left(g^{-1}\right) \phi\right)\left(k, k^{\prime}\right) \\
& \left.=(\delta \nu)^{-1}\left(a\left(k^{\prime} g^{-1}\right)\right) \pi_{\nu, \xi}(\phi)\left(k, g^{-1}\left[k^{\prime}\right]\right)\right) .
\end{aligned}
$$

Note that (A.56b) is the formal adjoint of (A.56 a), as it should. 


\section{Appendix B: The restricted dual of $\mathrm{SO}(1, \mathrm{~N})$}

Here we explicate some of the general results in appendix $\mathrm{A}$ for the case $\mathrm{SO}_{0}(1, N)$. These groups have split rank 1, i.e. the subgroup $A$ in the Iwasasa decomposition is one-dimensional. As a consequence the restricted dual is exhausted by the principal series proper and the discrete series; cuspidial principal series are absent. Further all unitary irreducible representations are multiplicity free, which gives rise to very explicit descriptions of their $\mathrm{SO}(N)$ content. Finally one can get explicit expressions for the coefficients of the spherical principal series in terms of simple special functions (generalized Legendre functions).

1. Group decompositions and orbits: The groups $\mathrm{SO}_{0}(1, N)$ are generalizations of the Lorentz group $(N=3)$ and the de-Sitter group $(N=4)$. They are connected and locally compact; the two-fold simply connected covering group of $\operatorname{SO}_{0}(1, N)$ is $\operatorname{Spin}(1, N)$. The natural action of $\mathrm{SO}_{0}(1, N)$ on $\mathbb{R}^{1, N}$ decomposes it into 6 types of orbits: the origin $\{x=$ $0\}$, the two-sheeted hyperboloids $\left\{x \cdot x=r, \pm x^{0}>0\right\}$, the one-sheeted hyperboloids (de-Sitter spaces) $\{x \cdot x=-r\}$ (with $r>0$ in both cases), and finally the cones $\{x \cdot x=$ $\left.0, \pm x^{0}>0\right\}$.

Both for the detailed description of these orbits and for the representation theory the Iwasawa decomposition $G=N A K$ is instrumental. For $G=\mathrm{SO}_{0}(1, N)$ it takes the following form: $K \simeq \mathrm{SO}(N)$ is the isotropy group of $q^{\uparrow}=(1,0, \ldots, 0)$. $A$ is the onedimensional subgroup generated by $a(\theta), \theta \in \mathbb{R}$, and $N$ is the $N-1$ dimensional subgroup generated by $n(t), t=\left(t_{1}, \ldots, t_{N-1}\right)^{T} \in \mathbb{R}^{N-1}$, where

$$
a(\theta)=\left(\begin{array}{cc|c}
\operatorname{ch} \theta & \operatorname{sh} \theta & \\
\operatorname{sh} \theta & \operatorname{ch} \theta & \\
\hline & & \mathbb{1}_{N-1}
\end{array}\right), \quad n(t)=\left(\begin{array}{cc|c}
1+\frac{1}{2} t^{2} & -\frac{t^{2}}{2} & t^{T} \\
\frac{t^{2}}{2} & 1-\frac{t^{2}}{2} & t^{T} \\
\hline t & -t & \mathbb{1}_{N-1}
\end{array}\right)
$$

with $t^{2}:=t_{1}^{2}+\ldots+t_{N-1}^{2}$. Observe that $n(t) n\left(t^{\prime}\right)=n\left(t+t^{\prime}\right)$ and $a(\theta) a\left(\theta^{\prime}\right)=a\left(\theta+\theta^{\prime}\right)$, so that $N \simeq \mathbb{R}^{N-1}$ and $A \simeq \mathbb{R}$. Each element $g \in G$ admits a unique decomposition $g=n a k$ with $n \in N, a \in A$, and $k \in K$. Since $g^{-1}=k^{-1} a^{-1} n^{-1}$ the same holds for a decomposition with the subgroups oppositely ordered, $G=K A N$. Let $\eta=\operatorname{diag}(1,-1, \ldots,-1)$ be the bilinear form on $\mathbb{R}^{1, N}$.

As before let $M$ denote the centralizer of $A$ in $K$. Clearly $M \simeq \mathrm{SO}(N-1)$, with $\mathrm{SO}(N-1)$ acting on the lower $(N-1) \times(N-1)$ block of the matrices. One has

$$
a(\theta) n(t) a(\theta)^{-1}=n\left(e^{\theta} t\right), \quad m n(t) m^{-1}=n(m t), \quad m \in M .
$$

This shows that as subgroups $A N=N A$ and that $N M$ is the semidirect product of $N \simeq \mathbb{R}^{N-1}$ with $M$. 
Explicit parameterizations of the various G-orbits in $\mathbb{R}^{1, N}$ can be obtained from the Iwasawa decomposition by letting it act on a reference vector of the orbit. For the upper sheet $\mathbb{H}_{N}=\left\{q \cdot q=1, q_{0}>0\right\}$ of the two-sheeted hyperboloid we take $q^{\uparrow}=(1,0, \ldots, 0)$ as the reference vector. The action of $G$ via the $N A K$ decomposition then gives the 'horispherical' coordinates on $\mathbb{H}_{N}$. Indeed, $n(t) a(\theta) q^{\uparrow}$ parameterizes a unique point $q=\left(q_{0}, \ldots, q_{N}\right)$ in $\mathbb{H}_{N}$,

$$
q_{0}=\operatorname{ch} \theta+\frac{1}{2} t^{2} e^{-\theta}, \quad q_{1}=\operatorname{sh} \theta+\frac{1}{2} t^{2} e^{-\theta}, \quad q_{i}=e^{-\theta} t_{i-1}, \quad i=2, \ldots, N
$$

and $\left(\theta, t_{1}, \ldots, t_{N-1}\right)$ are its horospherical coordinates. One can invert the transformation, in which case the product $n(t) a(\theta)$ viewed as a function of $q$ gives back the section $g_{s}(q)$ of Eq. (2.13). The isometry $\mathbb{H}_{N} \simeq \mathrm{SO}(1, N) / \mathrm{SO}(N)$ is likewise manifest. Similar descriptions - not needed here - exist for the cone $\left\{x \cdot x=0, x^{0}>0\right\}$ and the one-sheeted hyperboloid $\{q \cdot q=-1\}$.

Finally we note the relevant invariant measures. Let $d k$ denote the normalized Haar measure on $K=\operatorname{SO}(N)$. Set $d a=d \theta$ for $a=a(\theta) \in A$ which gives Haar measure on $A$. Then

$$
d(n a)=e^{-\theta(N-1)} d \theta d t_{1} \ldots d t_{N-1},
$$

is the left invariant measure on $N A$. The left invariance $d\left(n\left(t_{0}\right) a\left(\theta_{0}\right) n a\right)=d(n a)$ is easily checked from $a\left(\theta_{0}\right) n(t)=n\left(e^{\theta_{0}} t\right) a\left(\theta_{0}\right)$. The Haar measure on $G$ in the $N A K$ Iwasawa decomposition is then given by

$$
d g=d(n a) d k, \quad g \in N A K
$$

If elements $k \in K$ are decomposed according to $k=k(\vec{s}) m, m \in M, \vec{s} \in S^{N-1} \simeq K / M$, the normalized measures on $K, M$ and $S^{N-1}$ are related by

$$
d k=d S(\vec{s}) d m
$$

2. Dual and restricted dual of $\mathrm{SO}_{0}(1, N)$ : For $G=\operatorname{SO}_{0}(1, N)$ and $\operatorname{Spin}(1, N)$ the dual space (as a topological space) is known completely [2]. The lists in [40] and 26] are not quite complete and do not discuss the square integrability of the representations.

To illustrate the relation to the restricted dual $\widehat{G}_{r}$ we briefly sketch these results here. All unitary irreducible representations (UIR) come from those of the Lie algebra. For general $N$ the relevant UIR of the Lie algebra so $(1, N)$ have been classified by Ottoson [40] and Schwarz [47. Apart from the singlet $\pi_{0}$ there are three main types of UIR. For the harmonic analysis on $L^{2}\left(\mathrm{SO}_{0}(1, N)\right)$ only two of them are needed, the principal $\pi$ (princ) and the discrete series $\pi$ (disc). In addition there are complementary series $\pi$ (comp) (subdivided in 40, 47] into supplementary series and exceptional series). Each UIR is labeled by $r:=\operatorname{rank} \mathrm{SO}_{0}(1, N)=[(N+1) / 2]$ real parameters, $\left(\xi_{1}, \ldots, \xi_{r-1}, s\right)$. 
The $\xi_{i}$ are nonnegative and are either all integers or all halfintegers and are ordered $\xi_{1} \leq \xi_{2} \leq \ldots \leq \xi_{r-1}$. The remaining parameter $s$ can be real or complex. Depending on the value of $s$ the parameters $\xi$ are constrained by further conditions, which together with the value of $s$ specify to which series a given UIR belongs. For the principal series one has

$$
\pi \text { (princ) }: s=i \omega, \omega \geq 0, \quad \xi \in \widehat{\mathrm{SO}}(N-1),
$$

where the explicit description of the weights $\xi$ is given in Eq. (B.11) below. The general discussion of the principal series from Appendix A can be straightforwardly specialized to the case of $\mathrm{SO}_{0}(1, N)$. The $A$ homomorphisms $\nu=: \nu_{\omega}$ and $\delta$ are given by

$$
\begin{aligned}
\nu_{\omega}: A \rightarrow U(1), & \nu_{\omega}(a(\theta))=e^{i \omega \theta}, \quad \omega \geq 0, \\
\delta: A \rightarrow \mathbb{R}_{+}, & \delta(a(\theta))=e^{-\theta \frac{N-1}{2}} .
\end{aligned}
$$

For $N=2 r$ even there are also discrete series representations which enter the Plancherel decomposition. One has [27, 32]

$$
\pi^{ \pm}(\operatorname{disc}): \quad \pm s \leq \xi_{1} \leq \ldots \leq \xi_{r-1}, \quad \pm s \in \mathbb{N}, \xi_{i} \in \mathbb{N}
$$

where $\mathbb{N}$ are the positive integers and the $\pi^{ \pm}$series are labeled by the sign of $s$. The $(-s, \xi)$ representation is the mirror image of the $(s, \xi)$ representation in the following sense. Define a spatial reflection by $\theta\left(q_{0}, q_{1}, \ldots, q_{N}\right)=\left(q_{0},-q_{1}, \ldots, q_{N}\right)$, which induces an outer automorphism $g \mapsto \theta g \theta^{-1}$ of $\mathrm{SO}_{0}(1, N)$ (in the defining matrix representation). Then the mirror image $\pi_{\theta(s, \xi)}$ is defined by $\pi_{\theta(s, \xi)}(g)=\pi_{s, \xi}\left(\theta g \theta^{-1}\right)$; the result mentioned is that $\pi_{\theta(s, \xi)}$ is unitary equivalent to $\pi_{-s, \xi}$.

An explicit formula for the value of the quadratic Casimir on any of these UIR (in terms of their parameters) is known [27, 11].

As described in Appendix A.1 the Cartan subgroups are relevant for the harmonic analysis on $G$. In the case of $G=\mathrm{SO}_{0}(1, N)$, there is a single conjugacy class of Cartan subgroups when $N$ is odd and two when $N$ is even [57], p.188,212. In the notation of the previous section this arises because $\operatorname{dim} A=1$ in the Iwasawa decomposition $G=K A N$. In this case the number of conjugacy classes of $G$ is $2^{\operatorname{rank} K-\operatorname{rank} M}$. (Recall that $\operatorname{rank} \mathrm{SO}(N)=[N / 2]$, $\operatorname{rank} \mathrm{SO}(1, \mathrm{~N})=[(\mathrm{N}+1) / 2])$. Explicitly, if $H_{M}$ is the (up to conjugacy unique) Cartan subalgebra of $M$ one can adjoin either the generator of $A$ or the generator of $H_{K} / H_{M}$ (where $H_{K}$ is the up to conjugacy unique Cartan subalgebra of $K$ ) in order to obtain an abelian subgroup of $G$. For $N$ even both subgroups obtained in this way have the same dimension $N / 2=\operatorname{rankSO}(N)+1$. For $N$ odd $H_{M}$ and $H_{K}$ have the same dimension $\frac{N}{2}-1$ (a $2 \times 2$ block was needed to add a new Cartan generator). The compact abelian subalgebra $H_{M}=H_{K}$ is thus not maximal and only the noncompact Cartan subalgebra exists. According to the general discussion the Plancherel 
decomposition takes the form

$$
\begin{aligned}
L^{2}\left(\mathrm{SO}_{0}(1, N)\right) & =L^{2}\left(\mathrm{SO}_{0}(1, N)\right)_{\mathrm{disc}} \oplus \bigoplus_{\xi \in \widehat{M}} \int_{0}^{\infty} d \mu(\omega, \xi) \mathcal{L}_{\omega, \xi}, \\
L^{2}\left(\mathrm{SO}_{0}(1, N)\right)_{\text {disc }} & =\left\{\begin{array}{cc}
\{0\} & N \text { odd }, \\
\bigoplus_{\sigma \in \operatorname{disc}} L_{\sigma}^{2}\left(\mathrm{SO}_{0}(1, N)\right) & N \text { even },
\end{array}\right.
\end{aligned}
$$

where the sum in the discrete part ranges over the set in (B.9). An explicit formula for $d \mu(\omega, \xi)$ is known [27].

3. K-content of principal and discrete series: Restricted to the subgroup $K=\mathrm{SO}(N)$ the irreducible representations $\pi_{\sigma}$ of $\mathrm{SO}_{0}(1, N)$ decompose into a direct sum of irreducible representations $r_{\kappa}$ of $K$, each of which occurs with multiplicity at most one 10. The subset of $\widehat{K}_{\sigma} \subset \widehat{K}$ which occurs with nonzero (and hence unit) multiplicity is called the $K$ content of the representation. Here we describe it explicitly for the principal and the discrete series.

Recall from (B.7) that a principal series representation is labeled by a real parameter $\omega \geq 0$ and by a highest weight $\xi$ of $M=\mathrm{SO}(N-1)$. Explicitly the latter means

$$
\begin{aligned}
& N \text { even }: \quad \xi=\left(\xi_{1}, \ldots, \xi_{\frac{N-2}{2}}\right), \quad 0 \leq \xi_{1} \leq \ldots \leq \xi_{\frac{N-2}{2}}, \\
& N \text { odd }: \quad \xi=\left(\xi_{1}, \ldots, \xi_{\frac{N-1}{2}}\right), \quad\left|\xi_{1}\right| \leq \xi_{2} \leq \ldots \leq \xi_{\frac{N-1}{2}} .
\end{aligned}
$$

Here we used that UIR of the orthogonal groups are labeled by highest weights which are ordered sets $\left(m_{1}, \ldots, m_{r}\right)$, where $r=\operatorname{rank} \mathrm{SO}(N)$ equals $N / 2$ and $(N-1) / 2$ for $N$ even and odd, respectively. The $m_{i}$ are either all integer or all halfinteger and are subject to the constraints

$$
\begin{array}{rc}
\mathrm{SO}(2 r) & : \quad\left|m_{1}\right| \leq m_{2} \leq \ldots \leq m_{r}, \\
\mathrm{SO}(2 r+1) & : \quad 0 \leq m_{1} \leq \ldots \leq m_{r} .
\end{array}
$$

The singlet corresponds to $\kappa=(0, \ldots, 0)$; the symmetric traceless tensor representions have $\kappa=\left(0, \ldots, 0, m_{r}\right), m_{r} \in \mathbb{N}$. The mirror image $\theta \kappa$ of an UIR $\kappa$ is defined as follows. Let $\theta \in \mathrm{O}(N)$ be a reflection, i.e. $\theta^{2}=\mathbb{I}$, $\operatorname{det} \theta=-1$. Since $\theta k \theta^{-1} \in \mathrm{SO}(N)$ for all $k$, we can define a representation of $\mathrm{SO}(N)$ by $r_{\theta \kappa}(k):=r_{\kappa}\left(\theta k \theta^{-1}\right)$ called the mirror image of $\kappa$. It is again an irreducible highest weight representation and unitarily equivalent to $r_{\kappa^{\prime}}$, where

$$
\begin{aligned}
\mathrm{SO}(2 r) & : \quad \kappa^{\prime}=\left(-m_{1}, m_{2}, \ldots, m_{r}\right), \\
\mathrm{SO}(2 r+1) & : \quad \kappa^{\prime}=\left(m_{1}, m_{2}, \ldots, m_{r}\right)=\kappa .
\end{aligned}
$$

The $K=\mathrm{SO}(N)$ content $\pi_{\omega, \xi}$ can now be described explicitly

$$
\left.\pi_{\omega \xi}\right|_{K}=\bigoplus_{\kappa \in \widehat{K}_{\xi}} r_{\kappa}
$$


where each UIR of $K$ occurs with multiplicity precisely one and the subsets $\widehat{K}_{\xi} \subset \widehat{K}$ are characterized by [4, 47.

$$
\begin{array}{ll}
N \text { even }: \quad \kappa=\left(m_{1}, \ldots, m_{\frac{N}{2}}\right), \quad\left|m_{1}\right| \leq \xi_{1} \leq m_{2} \leq \ldots \leq \xi_{\frac{N-2}{2}} \leq m_{\frac{N}{2}} \\
N \text { odd }: \quad \kappa=\left(m_{1}, \ldots, m_{\frac{N-1}{2}}\right), & \left|\xi_{1}\right| \leq m_{1} \leq \xi_{2} \leq \ldots \leq \xi_{\frac{N-1}{2}} \leq m_{\frac{N-1}{2}}
\end{array}
$$

These are precisely the same conditions under which the UIR $\xi$ of $M=\mathrm{SO}(N-1)$ occurs (with unit multiplicity) in the restriction of $\left.r_{\kappa}\right|_{M}$ (see e.g. [3]). The result (B.11) thus exemplifies the general reciprocity rule mentioned in part $\mathrm{A}$ : if $\left.\pi_{\omega \xi}\right|_{K}$ contains $\kappa \in \widehat{K}$ (with unit multiplicity) then $\left.r_{\kappa}\right|_{M}$ contains $\xi \in \widehat{M}$ (with unit multiplicity).

Since the last label $m_{r}, r=\operatorname{rank} \mathrm{SO}(N)$, can be made arbitrarily large the subsets $K_{\xi}$ are always infinite. However with the exception of $\xi=0$ (the $M$-singlet) they never contain $\kappa=0$ (the $K$ singlet). The $K$ content of $\pi_{\omega, \xi=0}$ (the spherical principal series) is given by

$$
\widehat{K}_{\xi=0}=\left\{\left(0, \ldots, 0, m_{r}\right), \quad m_{r} \geq 0\right\} .
$$

As can be seen from (B.17) below the discrete series representations never contain a $K$ singlet. Irreducible representations containing a vector invariant under a compact subgroup are often called "class 1". One sees that among the representations of the restricted dual of $\mathrm{SO}_{0}(1, N)$ the only class 1 representations are those of the spherical principal series $\pi_{\omega, 0}$, and for them the class 1 property with respect to $M$ and with respect to $K$ is equivalent.

The $K$ content of the discrete series (B.9) comes out as follows

$$
\pi^{ \pm}(\operatorname{disc}): \quad \pm s \leq m_{1} \leq \xi_{1} \leq m_{2} \leq \ldots \leq \xi_{r-1} \leq m_{r}
$$

either by direct investigation of the Harish-Chandra characters [32, or by specialization of Blattner's formula [22. One sees from (B.13) that a discrete series representation never contains some $\kappa \in \widehat{K}$ together with its mirror image $\theta \kappa: m_{1}$ is strictly positive for the $\pi^{+}$series and strictly negative for the $\pi^{-}$series. Equivalently $\widehat{K}_{\pi^{+}} \cap \widehat{K}_{\pi^{-}}=\emptyset$. In particular the $\pi^{ \pm}$series never contain the $K$ singlet.

4. Harmonic analysis on $\mathbb{H}_{N}$ : Here we explicate the reduction of the harmonic analysis on $L^{2}\left(\mathrm{SO}_{0}(1, N)\right)$ to that on $L^{2}\left(\mathbb{H}_{N}\right)$, where $\mathbb{H}_{N}=\mathrm{SO}_{0}(1, N) / \mathrm{SO}(N), N \geq 2$, is the upper part of the two-sheeted hyperboloid in $\mathbb{R}^{N+1}$. The result will be the decomposition (A.26) of the quasiregular representation $\ell_{1}$ of $\mathrm{SO}_{0}(1, N)$ on $\mathbb{H}_{N}$. Since $K / M \simeq S^{N-1}$ all fiber spaces will be isometric to $L^{2}\left(S^{N-1}\right)$. The matrix elements (A.23) come out to be certain Legendre functions which are also generalized eigenfunctions of the LaplaceBeltrami operator on $\mathbb{H}_{N}$.

Let $-\Delta^{\mathbb{H}_{N}}$ be minus the Laplace-Beltrami operator on $\mathbb{H}_{N}$. Its spectrum is absolutely continuous and is given by the interval $\frac{1}{4}(N-1)^{2}+\omega^{2}, \omega>0$. There are several complete orthogonal systems of improper eigenfunctions. From a group theoretical viewpoint 
the most convenient system are the 'principal plane waves' $\epsilon_{\omega, p}(q)$ (see [56] and the references therein) labeled by $\omega>0$ and a 'momentum' vector $\vec{p} \in S^{N-1}$. Parameterizing $q=\left(\xi, \sqrt{\xi^{2}-1} \vec{s}\right)$, they read

$$
\epsilon_{\omega, p}(q):=\left[\xi-\sqrt{\xi^{2}-1} \vec{s} \cdot \vec{p}\right]^{-\frac{1}{2}(N-1)-i \omega} .
$$

The completeness and orthogonality relations take the form

$$
\begin{aligned}
\int d \gamma_{Q}(q) \epsilon_{\omega, p}(q)^{*} \epsilon_{\omega^{\prime}, p^{\prime}}(q) & =d(\omega)^{-1} \delta\left(\omega-\omega^{\prime}\right) \delta\left(\vec{p}, \vec{p}^{\prime}\right), \\
\int_{0}^{\infty} d \omega d(\omega) \int_{S^{N-1}} d S(p) \epsilon_{\omega, p}(q)^{*} \epsilon_{\omega, p}\left(q^{\prime}\right) & =\delta\left(q, q^{\prime}\right)
\end{aligned}
$$

where $\delta\left(q, q^{\prime}\right)$ and $\delta\left(\vec{p}, \vec{p}^{\prime}\right)$ are the normalized delta distributions with respect to the invariant measures $d \gamma_{Q}(q)$ and $d S(p)$ on $\mathbb{H}_{N}$ and $S^{N-1}$, respectively. In terms of the coordinates $(\xi, \vec{s})$ the former reads

$$
\int d \gamma_{Q}(q)=\int_{1}^{\infty} d \xi\left(\xi^{2}-1\right)^{N / 2-1} \int_{S^{N-1}} d S(\vec{s})
$$

The spectral weight is determined by the Harish-Chandra c-function for $\operatorname{SO}_{0}(1, N)$ and is given by is

$$
d(\omega)=\frac{1}{(2 \pi)^{N}}\left|\frac{\Gamma\left(\frac{N-1}{2}+i \omega\right)}{\Gamma(i \omega)}\right|^{2} .
$$

The main virtue of these functions is their simple transformation law under $\mathrm{SO}_{0}(1, N)$, see e.g. Appendix A of [12. It characterizes the spherical principal unitary series $\pi_{\omega, 0}, \omega \geq 0$, of $\mathrm{SO}_{0}(1, N)$, where $\pi_{\omega, 0}$ and its complex conjugate are unitary equivalent (see e.g. 56], Sections 9.2.1 and 9.2.7). The orthogonality and completeness relations (B.19) amount to the decomposition (A.26) of the quasi-regular representation $\ell_{1}$ on $L^{2}\left(\mathbb{H}_{N}\right)$.

Spectral projectors $E_{I}$ commuting with $\ell_{1}$ are defined in terms of their kernels $E_{I}\left(q \cdot q^{\prime}\right)$, $I \subset \mathbb{R}_{+}$by

$$
\begin{aligned}
& E_{I}\left(q \cdot q^{\prime}\right):=\int_{I} d \omega d(\omega) \int_{S^{N-1}} d S(p) \epsilon_{\omega, p}(q)^{*} \epsilon_{\omega, p}\left(q^{\prime}\right) \\
& \int d \Omega\left(q^{\prime}\right) E_{I}\left(q \cdot q^{\prime}\right) E_{J}\left(q^{\prime} \cdot q^{\prime \prime}\right)=E_{I \cap J}\left(q \cdot q^{\prime \prime}\right)
\end{aligned}
$$

Combined with the completeness relation in (B.19) this shows that the spectrum of $-\Delta^{\mathbb{H}_{N}}$ is absolutely continuous.

A complete orthogonal set of real eigenfunctions of $-\Delta_{\mathbb{H}_{N}}$ is obtained by taking the $d S(p)$ average of the product of $\epsilon_{\omega, p}(q)$ with some spherical harmonics on the $p$-sphere. 
This amounts to a decomposition in terms of $\mathrm{SO}^{\uparrow}(N)$ UIR where the 'radial' parts of the resulting eigenfunctions are given by Legendre functions. Using the normalization and the integral representation from (20] p. 1000) one has in particular

$$
\int_{S^{N-1}} d S(p) \epsilon_{\omega, p}(q)=(2 \pi)^{N / 2}\left(\xi^{2}-1\right)^{\frac{1}{4}(2-N)} \mathcal{P}_{-1 / 2+i \omega}^{1-N / 2}(\xi) .
$$

As a check on the normalizations one can take the $\xi \rightarrow 1^{+}$limit in (B.23). The limit on the rhs is regular and gives $2 \pi^{N / 2} / \Gamma(N / 2)$, which equals the area of $S^{N-1}$ as required by the limit of the lhs. Denoting the set of real scalar spherical harmonics by $Y_{\ell m}(k)$, $\ell \in \mathbb{N}_{0}, m=0, \ldots, d_{\ell}-1$, with $d_{\ell}=(2 \ell+N-2)(\kappa+N-3) ! /(\ell !(N-2) !)$ we set

$$
\begin{aligned}
E_{\omega, \ell m}(q):= & \int d S(p) Y_{\ell m}(p) \epsilon_{\omega, p}(q) \\
= & n_{\ell}(\omega) Y_{\ell m}(\vec{s})\left(\xi^{2}-1\right)^{\frac{1}{4}(2-N)} \mathcal{P}_{-1 / 2+i \omega}^{1-N / 2-\ell}(\xi), \quad \text { with } \\
& n_{0}(\omega)=(2 \pi)^{N / 2}, \quad n_{\ell}(\omega)=(2 \pi)^{N / 2}\left(\prod_{j=0}^{\ell-1}\left[\omega^{2}+\left(\frac{N-1}{2}+j\right)^{2}\right]\right)^{1 / 2}, \ell \geq 1 .
\end{aligned}
$$

The expression (B.24 $)$ is manifestly real, the equivalence to $(\mathbb{B} .24 \mathrm{~b})$ can be seen as follows: from (B.19), (B.20), and the orthogonality and completeness of the spherical harmonics one readily verifies that both $(\mathbb{B} .24 \mathrm{a})$ and $(\mathbb{B} .24 \mathrm{~b})$ satisfy

$$
\begin{aligned}
\int d \Omega(q) E_{\omega, \ell m}(q)^{*} E_{\omega^{\prime}, \ell^{\prime} m^{\prime}}(q) & =d(\omega)^{-1} \delta\left(\omega-\omega^{\prime}\right) \delta_{\ell, \ell^{\prime}} \delta_{m, m^{\prime}}, \\
\int_{0}^{\infty} d \omega d(\omega) \sum_{\ell, m} E_{\omega, \ell m}(q)^{*} E_{\omega, \ell m}\left(q^{\prime}\right) & =\delta\left(q, q^{\prime}\right) .
\end{aligned}
$$

Further both $(\mathbb{B} .24 \mathrm{a})$ and $(\bar{B} .24 \mathrm{~b})$ transform irreducibly with respect to the real $d_{\ell}$ dimensional matrix representation of $\mathrm{SO}^{\uparrow}(N)$ carried by the spherical harmonics. Hence they must coincide. A drawback of the functions (B.24) is that the $\vec{k}$ integration spoils the simple transformation law of the $\epsilon_{\omega, p}$ under $\mathrm{SO}(1, N)$. The transformation law can now be inferred from the addition theorem

$$
\sum_{\ell, m} E_{\omega, \ell m}(q) E_{\omega, \ell m}\left(q^{\prime}\right)=(2 \pi)^{N / 2}\left[\left(q \cdot q^{\prime}\right)^{2}-1\right]^{\frac{1}{4}(2-N)} \mathcal{P}_{-1 / 2+i \omega}^{1-N / 2}\left(q \cdot q^{\prime}\right)
$$

For example for $q^{\prime}=g q^{\uparrow}$ this describes the transformation of the $\mathrm{SO}^{\uparrow}(N)$ singlet $E_{\omega, 0,0}(q)$ under $g \in \mathrm{SO}(1, N)$. 


\section{Appendix C: The amenable case $\operatorname{ISO}(\mathrm{N})$}

Here we outline counterparts of our main results for the coset space $\operatorname{ISO}(N) / \mathrm{SO}(N) \simeq$ $\mathbb{R}^{N}$. This coset can be viewed as the flat space limit of the hyperboloid $\mathrm{SO}_{0}(1, N) / \mathrm{SO}(N)$. The underlying Euclidean group $\operatorname{ISO}(N)$ is noncompact but amenable; in accordance with the general picture [38] the generalized spin systems turn out to have a unique non-normalizable ground state.

The group $\operatorname{ISO}(N)$ is the semi-direct product of the amenable groups $\mathrm{SO}(N)$ and $\mathbb{R}^{N}$, and hence is itself amenable, 9, Lemma 18.3.7. As the defining representation one can take a subgroup of $N+1 \times N+1$ matrices acting on vectors $(x, 1)^{T}$ in $\mathbb{R}^{N+1}$

$$
g(k, a)=\left(\begin{array}{ll}
k & a \\
0 & 1
\end{array}\right), \quad g(k, a)\left(\begin{array}{l}
x \\
1
\end{array}\right)=\left(\begin{array}{c}
k x+a \\
1
\end{array}\right), \quad k \in \operatorname{SO}(N), \quad a, x \in \mathbb{R}^{N}
$$

The composition law is $g\left(k_{1}, a_{1}\right) g\left(k_{2}, a_{2}\right)=g\left(k_{1} k_{2}, a_{1}+k_{1} a_{2}\right)$, which gives $g(k, a)^{-1}=$ $g\left(k^{-1},-k^{-1} a\right)$ and $g(k, a)=g(e, a) g(k, 0)=g(k, 0) g\left(e, k^{-1} a\right)$. Haar measure on $\operatorname{ISO}(N)$ is $d k d a$, where $d k$ is the normalized Haar measure on $\mathrm{SO}(N)$ and $d a$ Lebesgue measure on $\mathbb{R}^{N}$.

We shall need a section $g_{s}: \mathbb{R}^{N} \rightarrow \operatorname{ISO}(N)$ such that $x=g_{s}(x) x^{\uparrow}$, where $x^{\uparrow}=(0,1)^{T}$ is fixed by the rotation subgroup. An obvious choice is

$$
g_{s}(x)=\left(\begin{array}{ll}
e & x \\
0 & 1
\end{array}\right), \quad k_{s}(g(k, a), x)=k^{-1}
$$

for which the cocycle $k_{s}$ in (2.12) is independent of $x$ and $a$.

The configuration manifold is $\mathcal{M}=Q^{\nu}=\mathbb{R}^{N \nu}$, the state space is $L^{2}(\mathcal{M})=L^{2}\left(\mathbb{R}^{N \nu}\right)$, and $\operatorname{ISO}(N)$ acts on it via the $\nu$-fold inner product of the left quasiregular representation $\ell_{1}$. Explicitly

$$
\left[\ell_{\mathcal{M}}(k, a) \psi\right](x)=\psi\left(k^{-1}(x-a)\right), \quad x=\left(x_{1}, \ldots, x_{\nu}\right)
$$

where we wrote $\ell_{\mathcal{M}}(k, a)$ for $\ell_{\mathcal{M}}(g(k, a))$. A transfer operator $\mathbf{T}$ in the sense of definition 2.1 is described by a kernel $T: \mathbb{R}^{N \nu} \times \mathbb{R}^{N \nu} \rightarrow \mathbb{R}_{+}$, which is symmetric, continuous, pointwise strictly positive, and subject to the condition (2.2). The invariance $\ell_{\mathcal{M}} \circ \mathbf{T}=$ $\mathbf{T} \circ \ell_{\mathcal{M}}$ translates into $T(k(x+a), k(y+a))=T(x, y)$, for all $k \in \mathrm{SO}(N)$ and $a \in \mathbb{R}^{N}$. To exploit this symmetry we proceed as in Section 2 and switch to a model of $L^{2}(\mathcal{M})$ where $\ell_{\mathcal{M}}$ acts via right multiplication on a single group-valued argument.

Recall that $\mathcal{M}_{r}=(G \times \mathcal{N}) / d(K)$ and that the isometry to $\mathcal{M}$ is constructed from the $\operatorname{map} \tilde{\phi}: \mathcal{M} \rightarrow \operatorname{ISO}(N) \times \mathcal{N}, \mathcal{N} \simeq \mathbb{R}^{N(\nu-1)}$

$$
\begin{aligned}
\tilde{\phi}\left(x_{1}, \ldots, x_{\nu}\right) & =\left(g_{s}\left(x_{1}\right)^{-1}, x_{2}-x_{1}, \ldots, x_{\nu}-x_{1}\right), \\
\tilde{\phi}\left(g^{-1} x_{1}, \ldots, g^{-1} x_{\nu}\right) & =\left(g_{s}\left(x_{1}\right)^{-1} g, x_{2}-x_{1}, \ldots, x_{\nu}-x_{1}\right) .
\end{aligned}
$$


As expected the group acts on the image points by right multiplication on the first argument. Since $g\left(k_{0}, 0\right) g_{s}\left(x_{1}\right)^{-1} g(k, a)=g\left(k_{0} k,-k_{0}\left(x_{1}-a\right)\right)$ the left $\mathrm{SO}(N)$ invariant functions on $\operatorname{ISO}(N) \times \mathcal{N}$ are characterized by

$$
\psi_{r}\left(g_{s}\left(e,-k^{-1}\left(x_{1}-a\right)\right), k^{-1} n\right)=\psi_{r}\left(g\left(k,-x_{1}+a\right), n\right)
$$

Effectively the functions $\psi_{r}$ thus project onto functions on $Q \times \mathcal{N}$, however at the price of a more complicated group action. In fact $\psi_{s}(x, n):=\psi_{r}(g(e,-x), n)$ defines an element of $L^{2}\left(\mathcal{M}_{s}\right)$ with the group action $\left[\ell_{s}(k, a) \psi_{s}\right](x, n)=\psi_{s}\left(k^{-1}(x-a), k^{-1} n\right)$.

The unitary irreducible representations (UIR) entering the decomposition of the regular representation of $\operatorname{ISO}(N)$ on $L^{2}(\operatorname{ISO}(N))$ can be described as follows (Gross and Kunze, [21). For $0 \neq \nu \in \mathbb{R}^{N}$ let $K_{\nu}$ be the isotropy group of $\nu$ in $K=\mathrm{SO}(N)$. Then each $K_{\nu}$ is conjugate to $M:=\mathrm{SO}^{\uparrow}(N-1)$, defined as the subgroup leaving $e_{1}=(1,0, \ldots, 0)^{T} \in \mathbb{R}^{N}$ invariant. Let $m \mapsto r_{\xi}(m), \xi \in \widehat{M}$, be an irreducible representation of $M$ on $V_{\xi}$ and consider (as in Eq. (A.43)

$$
\begin{aligned}
& L_{\xi}^{2}(K)=\left\{f \in L^{2}\left(K, V_{\xi}\right), f(m k)=r_{\xi}(m) f(k), m \in M\right\} \\
& \left(f_{1}, f_{2}\right):=\int_{K} d k\left\langle f_{1}(k), f_{2}(k)\right\rangle_{V_{\xi}} .
\end{aligned}
$$

On $L_{\xi}^{2}(K)$ define a unitary representation by

$$
\left[\pi_{\nu, \xi}\left(k_{0}, a_{0}\right) f\right](k)=e^{i \nu \cdot k a_{0}} f\left(k k_{0}\right)
$$

This is well-defined because $\pi_{\nu, \xi}$ commutes with the left regular representation of $M$. In particular $\left[\pi_{\nu, \xi}\left(k_{0}, a_{0}\right) f\right](m k)=r_{\xi}(m)\left[\pi_{\nu, \xi}\left(k_{0}, a_{0}\right) f\right](k)$. Moreover [21]:

- $\pi_{\nu, \xi}$ is irreducible for all $0 \neq \nu \in \mathbb{R}^{N}$ and $\xi \in \widehat{M}$.

- Every infinite dimensional unitary representation is equivalent to some such $\pi_{\nu, \xi}$.

- Given $0 \neq \nu, \nu^{\prime} \in \mathbb{R}^{N}$ and $\xi, \xi^{\prime} \in \widehat{M}$, the representations $\pi_{\nu, \xi}$ and $\pi_{\nu^{\prime}, \xi^{\prime}}$ are equivalent if and only if first $\nu$ and $\nu^{\prime}$ belong to the same $\mathrm{SO}(N)$ orbit and second $\xi$ and $\xi^{\prime}$ are equivalent under the identification of $K_{\nu}$ with $K_{\nu^{\prime}}$.

These representations constitute the principal series of $\operatorname{ISO}(N)$, the subset with $\xi=0$ ( $M$-singlets) is called the spherical principal series. We now fix a representative from each equivalence class as follows. If $\nu \cdot \nu=\nu^{\prime} \cdot \nu^{\prime}=\omega^{2}, \omega \in \mathbb{R}_{+}$, then by abuse of notation we denote the $N$-tuple $(0, \ldots, 0, \omega)^{T} \in \mathbb{R}^{N}$ also by $\omega$. In this case we write $K_{\omega}=\mathrm{SO}^{\uparrow}(N-1)$ for $K_{\nu}$ and $\pi_{\omega, \xi}$ for $\pi_{\nu, \xi}$. The $K$-content of these representations is the 
same as that of the corresponding principal series representations of $\mathrm{SO}_{0}(1, N)$, as their restrictions to $K$ coincide. This gives

$$
\left.\pi_{\omega, \xi}\right|_{K}=\bigoplus_{\ell \in \widehat{K}_{\xi}} r_{\ell}
$$

with $\widehat{K}_{\xi} \subset \widehat{K}$ as in (B.15).

The finite dimensional representations of $\operatorname{ISO}(\mathrm{N})$, obtained from the irreducible representations of $\mathrm{SO}(\mathrm{N})$ by representing the abelian normal subgroup of translations trivially are not in the support of the regular representation.

So the Plancherel decomposition takes the form

$$
\begin{aligned}
L^{2}(\operatorname{ISO}(N)) & =\int_{0}^{\infty} d \omega d(\omega) \bigoplus_{\xi \in \widehat{M}} \operatorname{dim} V_{\xi} \mathcal{L}_{\omega, \xi} \otimes \check{\mathcal{L}}_{\omega, \check{\xi}}, \\
\rho \times \ell & =\int_{0}^{\infty} d \omega d(\omega) \bigoplus_{\xi \in \widehat{M}} \operatorname{dim} V_{\xi} \pi_{\omega, \xi} \otimes \check{\pi}_{\omega, \check{\xi}}
\end{aligned}
$$

with

$$
d(\omega)=\frac{\omega^{N-1}}{(2 \pi)^{N}} \frac{2 \pi^{N / 2}}{\Gamma(N / 2)},
$$

(which can be viewed as the squared inverse of the Harish-Chandra $c$ function for $\operatorname{ISO}(N)$; see [52] for $N=2$ ). The formulae for the harmonic analysis and synthesis will be given below. In relation to (C.9) the singlet representation deserves special consideration. Since $\operatorname{ISO}(N)$ is amenable the singlet must weakly be contained in (equivalently: lie in the support of) the regular representation (see e.g. 9], Prop.18.3.6; and Definitions 18.3.1, 18.1.7). By definition this support is the restricted dual of the locally compact group under consideration, here $\operatorname{ISO}(N)$. As a matter of fact 9, 18.8.4, it also coincides with the carrier of the Plancherel measure. The upshot is that the limit $\lim _{\omega \rightarrow 0} \pi_{\omega, \xi=0}$ is weakly contained in the decomposition (C.9) and coincides with the singlet representation of $\operatorname{ISO}(N)$. We shall write $\pi_{00}$ for it.

To every $\phi \in L^{1}(\operatorname{ISO}(N))$ one can asign a compact operator as its Fourier transform

$$
\widehat{\phi}(\nu, \xi)^{\dagger}=\int d k d a \phi(k, a) \pi_{\nu, \xi}(k, a)=\pi_{\nu, \xi}(\phi)
$$

and for $\phi \in\left(L^{1} \cap L^{2}\right)(\operatorname{ISO}(N))$ the image is a Hilbert-Schmidt operator for almost all $(\omega, \xi)$ with respect to the Plancherel measure. Since the latter is for fixed $\xi$ absolutely continuous with respect to Lebesgue measure on $\mathbb{R}_{+}$, the Hilbert-Schmidt property will hold for almost all $\omega>0$ with respect to the Lebesgue measure. Indeed, $\pi_{\omega, \xi}(\phi)$ can be 
realized explicitly as an integral operator on $L_{\xi}^{2}(K)$. Repeating the steps in Appendix A.8 one finds

$$
\begin{aligned}
{\left[\pi_{\nu, \xi}(\phi) f\right](k) } & =\int d k^{\prime} \pi_{\nu, \xi}(\phi)\left(k, k^{\prime}\right) f\left(k^{\prime}\right), \\
\pi_{\nu, \xi}(\phi)\left(k, k^{\prime}\right) & =\int_{M \times \mathbb{R}^{N}} d m d a \phi\left(k^{-1} m k^{\prime}, a\right) e^{i \nu \cdot k a} r_{\xi}(m) .
\end{aligned}
$$

The formula for the Fourier synthesis reads

$$
\phi(k, a)=\int_{0}^{\infty} d \omega d(\omega) \sum_{\xi \in \widehat{M}} \operatorname{dim} V_{\xi} \operatorname{Tr}\left[\widehat{\phi}(\omega, \xi) \pi_{\omega, \xi}(k, a)\right]
$$

Formally this can be verified by evaluating the trace in terms of a kernel of the form (C.12) and freely exchanging the order of integrations. For a proof see [21].

With these preparations at hand we can proceed with the group theoretical decomposition of the Hilbert space $L^{2}\left(\mathcal{M}_{r}\right)$ and of standard invariant selfadjoint operators $\mathbf{A}$ acting on it. The constructions of Sections 2 and 3 carry over with minor modifications; mainly to fix the notations we run through the main steps. Proposition 2.2 remains valid with the Plancherel measure from (C.9) substituted and with the $K$-content from (C.8). We use pairs $\sigma=(\omega, \xi)$ and $\check{\sigma}=(\omega, \check{\xi})$ to label the representations and their conjugates. Proposition 3.4 likewise carries over and provides the decomposition of the operators. We write

$$
\begin{aligned}
L^{2}\left(\mathcal{M}_{r}\right) & =\int_{0}^{\infty} d \omega d(\omega) \bigoplus_{\xi \in \widehat{M}} \operatorname{dim} V_{\xi} \mathcal{L}_{\omega \xi}^{2}\left(\mathcal{M}_{r}\right), \\
\mathbf{A} & =\int_{0}^{\infty} d \omega d(\omega) \bigoplus_{\xi \in \widehat{M}} \operatorname{dim} V_{\xi}\left(\mathbb{I} \otimes \check{\mathbf{A}}_{\omega \check{\xi}}\right),
\end{aligned}
$$

for the respective decompositions. The fiber spaces $\mathcal{L}_{\omega \xi}^{2}\left(\mathcal{M}_{r}\right)$ are isometric to $\mathcal{L}_{\omega \xi} \otimes$ $\check{\mathcal{L}}_{\omega \check{\xi}} \otimes L^{2}(\mathcal{N})$ and $\mathbb{I} \otimes \check{\mathbf{A}}_{\omega \check{\xi}}$ acts for almost all $\omega>0$ as a bounded linear and selfadjoint operator on these fiber spaces.

The spectral problems of $\mathbf{A}$ and $\mathbf{A}_{\omega \xi}$ can now be related as in Sections 3.3 and 3.4. We maintain the definitions of the generalized eigenspaces $\mathcal{E}_{\lambda, \omega \xi}(\mathbf{A})$ and $\mathcal{E}_{\lambda}\left(\mathbf{A}_{\omega \xi}\right)$, in Eqs. (3.41) and (3.61), respectively. Then the map

$$
\begin{aligned}
& \tau_{v, \omega \xi}: \mathcal{E}_{\lambda}\left(\mathbf{A}_{\omega \xi}\right) \rightarrow \mathcal{E}_{\lambda, \omega \xi}(\mathbf{A}), \quad f \mapsto \tau_{v, \omega \xi}(f), \\
& \tau_{v, \omega \xi}(f)(k, a, n)=\int_{K} d k^{\prime} f\left(n, k^{\prime}\right)^{*}\left[\pi_{\omega \xi}(k, a) v\right]\left(k^{\prime}\right), \quad v \in L_{\xi}^{2}(K),
\end{aligned}
$$

again provides an isometry onto its image. In the second line we used the realization of $\mathcal{L}_{\omega, \xi}$ as $L_{\xi}^{2}(K)$ in (C.6). The intertwining properties $\tau_{v, \omega \xi}(f)\left(g g_{0}, n\right)=\tau_{\pi_{\omega \xi}\left(g_{0}\right) v, \omega \xi}(f)(g, n)$ and $\mathbf{A} \tau_{v, \omega \xi}(f)=\tau_{v, \omega \xi}\left(\mathbf{A}_{\omega \xi} f\right)$ remain valid. Then Propositions 3.6 and 3.8 carry over. 
Major modifications however occur in the structure of the ground state sector of a transfer operator $\mathbf{T}$. Its fiber operators $\mathbf{T}_{\omega \xi}$ can be realized as integral operators on $L_{\xi}^{2}(K)$ with the following kernel

$$
\mathcal{T}_{\omega \xi}\left(n, n^{\prime} ; k, k^{\prime}\right)=\int_{M \times \mathbb{R}^{N}} d m d a \mathcal{T}\left(k^{-1} m k^{\prime}, a, n^{\prime}, n\right) e^{i \omega e_{1} \cdot k a} r_{\xi}(m)
$$

For these integral operators a counterpart of Proposition 4.1 holds: the operators $\mathbf{T}_{\omega \xi}$ : $L_{\xi}^{2}(\mathcal{N}) \rightarrow L_{\xi}^{2}(\mathcal{N})$ are bounded for all (not almost all) $\omega \geq 0, \xi \in \widehat{M}$. Their norms are continuous functions of $\omega$ and obey

$$
\left\|\mathbf{T}_{\omega \xi}\right\| \leq\left\|\mathbf{T}_{00}\right\| \quad \text { for all } \xi \in \widehat{M}, \omega \geq 0,
$$

where the inequality is strict unless $\xi=0$ and $\omega=0$. Further $\mathbf{T}_{00}$ is a transfer operator in the sense of Definition 2.1.

This result entails that all the generalized eigenspaces $\mathcal{E}_{\|\mathbf{T}\|}\left(\mathbf{T}_{\omega \xi}\right), \omega>0, \xi \in \widehat{M}$, must be empty. The remaining $\mathcal{E}_{\|\mathbf{T}\|}\left(\mathbf{T}_{00}\right)$ coincides with the ground state sector $\mathcal{G}\left(\mathbf{T}_{00}\right)$ of $\mathbf{T}_{00}$. Provided the map (C.15) is defined also for $\omega=0, \xi=0$ it asigns to every generalized ground state $f \in \mathcal{G}\left(\mathbf{T}_{00}\right)$ a generalized ground state $\tau_{v, 00}(f)$ of $\mathbf{T}$. Moreover by forming linear combinations $\sum_{i} \tau_{v_{i}, 00}\left(f_{i}\right)$ one can generate a dense set in $\mathcal{G}(\mathbf{T})$. Since the singlet representation $\pi_{00}$ is one-dimensional, while all the $\pi_{\omega \xi}, \omega>0$, are infinite dimensional, the family of maps $\tau_{v, \omega 0}$ is of course not continuous for $\omega \rightarrow 0$. However from (C.16) one can verify directly that every eigenfunction of $\mathbf{T}_{00}$, viewed as a function on $G / K \times \mathcal{N} / d_{\mathcal{N}}(K)$ constant in the first argument, is also a generalized eigenfunction of $\mathbf{T}$. Indeed the counterpart of C.15 for the singlet representation is simply $\tau_{00}(f)(e, n):=\int d k f(n, k)^{*}$, as the function $v$ is constant and can be omitted. Then $\left[\mathbf{T} \tau_{00}(f)\right](e, n)=\tau_{00}\left(\mathbf{T}_{00} f\right)(e, n)$, where the relevant kernel $\int d k d a \mathcal{T}\left(k, a, n, n^{\prime}\right)$ is symmetric in $n$ and $n^{\prime}$. It follows that linear combinations $\sum_{i} c_{i} \tau_{00}\left(f_{i}\right), c_{i} \in \mathbb{C}$, generate a dense subspace of $\mathcal{G}(\mathbf{T})$. In particular all generalized ground states of $\mathbf{T}$ are functions on $G / K \times \mathcal{N} / d_{\mathcal{N}}(K)$ constant in the first argument. This means $\tau_{00}: \mathcal{G}\left(\mathbf{T}_{00}\right) \rightarrow \mathcal{G}(\mathbf{T})$ is an isometry. Whenever $\|\mathbf{T}\|$ is an eigenvalue of $\mathbf{T}_{00}$ both $\mathcal{G}\left(\mathbf{T}_{00}\right)$ and $\mathcal{G}(\mathbf{T})$ are onedimensional. Viewed as an element of $\mathcal{G}(\mathbf{T})$ however the wave function is not normalizable as the infinite volume of $\operatorname{ISO}(N)$ is overcounted. In summary, we arrive at

Theorem C.1 Let $\mathbf{T}$ be a transfer operator on $L^{2}\left(\mathbb{R}^{N \nu}\right)$ commuting with the unitary representation (C.3) of $\operatorname{ISO}(N)$. Let $\mathcal{G}_{\omega \xi}(\mathbf{T})$ denote the space of generalized ground states whose elements transform equivariantly according to $\pi_{\omega \xi}, \omega \geq 0, \xi \in \widehat{\mathrm{SO}}(N-1)$, and let $\mathbb{I} \otimes \check{\mathbf{T}}_{\omega \check{\xi}}$ be the component of $\mathbf{T}$ in the fiber $\pi_{\omega \xi}$. Then:

(a) $\mathcal{G}_{\omega \xi}(\mathbf{T})$ is empty unless $\omega=0, \xi=0$, where $\pi_{00}$ is the singlet representation.

(b) $\mathcal{G}(\mathbf{T})$ can isometrically be identified with $\mathcal{G}\left(\mathbf{T}_{00}\right)$ and is generated by rotationally invariant functions of $x_{2}-x_{1}, \ldots, x_{\nu}-x_{1}$. Whenever $\|\mathbf{T}\|$ is an eigenvalue of $\mathbf{T}_{00}$ the transfer operator $\mathbf{T}$ has a unique ground state, which is up to a phase an a.e. strictly positive function of the above type. 


\section{References}

[1] M. Atiyah and W. Schmid, A geometric construction of the discrete series for semisimple Lie groups, Inventiones Math. 42 (1977) 1-62.

[2] M.W. Baldoni-Silva and D. Barbasch, The unitary spectrum of real rank one groups, Inv. Math. 72 (1983) 27.

[3] A. Barut and R. Rączka, Theory of group representations and applications, World Scientific, Singapore 1986.

[4] B. Bekka, Amenable unitary representations of locally compact groups, Inv. Math. 100 (1990) 383.

[5] B. Bekka, P. de la Harpe and A. Valette, Kazhdan groups, forthcoming book, see http://www.mmas.univ-metz.fr/ bekka.

[6] A. Bohm and M. Gadella, Dirac Kets, Gamow Vectors and Gel'fand Triplets. The Rigged Hilbert Space Formulation of Quantum Mechanics, Springer-Verlag, Berlin etc. 1989.

[7] A. Comtet, On the Landau levels on the hyperbolic plane, Ann. Physics 173 (1987) 185.

[8] A. Davies, A. MacFarlane and J. van Holten, The quantum-mechanical supersymmetric O(3) and O(2,1) sigma-models, Nucl. Phys. B216 (1983) 493.

[9] J. Dixmier, $C^{*}$-Algebras, North Holland, Amsterdam etc. 1977.

[10] J. Dixmier, Sur les représentations de certains groupes orthogonaux, Compt. Rend. 250 (1960) 3263 .

[11] V. Dobrev, G. Mack, V. Petkova, S. Petrova, and I. Todorov, Harmonic analysis on the $n$-dimensional Lorentz group and its applications to conformal quantum field theory, Lecture notes in physics, Vol.63, Springer, Berlin etc. 1977.

[12] A. Duncan, M. Niedermaier and E. Seiler, Vacuum orbit and spontaneous symmetry breaking in noncompact nonlinear sigma-models, Nucl. Phys. 720 (2005) 235-288 hep-th/0405163.

[13] K. B. Efetov, Supersymmetry and theory of disordered metals, Adv. Phys. 32 (83) 53.

[14] K. B. Efetov, Supersymmetry in Disorder and Chaos, Cambridge University Press, Cambridge, U.K. 1997.

[15] G. Folland, A course in abstract harmonic analysis, CRC Press, Boca Raton etc. 1995.

[16] S. Fulling, Absence of trivial subrepresentations from tensor products of unitary representations of pseudo-orthogonal groups, J. Math. Phys. 15 (1974) 1567.

[17] I. M. Gel'fand and N. Y. Vilenkin, Generalized Functions, Vol. IV, Academic Press, New York 1964. 
[18] J. Glimm, Type I $C^{*}$-Algebras, Ann. Math. 73 (1961) 572.

[19] J. Glimm and A. Jaffe, Quantum Physics, Springer-Verlag, Berlin etc, 1987.

[20] I. Gradshteyn and I. Ryzhik, Table of integrals and products, Academic Press, New York and London 1980.

[21] K. Gross and R. Kunze, Fourier decompositions of certain representations, in: W. Boothby and G. Weiss (Eds), Symmetric Spaces, Marcel Decker, New York, 1972 (pp. 119-139).

[22] H. Hecht and W. Schmid, A proof of Blattner's conjecture, Inv. Math. 31 (1975) 129.

[23] S. Helgason, Differential geometry and symmetric spaces, Academic Press, New York 1962.

[24] S. Helgason, Geometric analysis on symmetric spaces, American Mathematical Society, Providence, R.I. 1991.

[25] R. Herb and J. Wolf, The Plancherel theorem for general real semi-simple Lie groups, Compositio Math. 57 (1986) 271.

[26] T. Hirai, On irreducible representations of the Lorentz group of $n$-th order, Proc. Japan Acad. 38 (1962) 258.

[27] T. Hirai, The Plancherel formula for the Lorentz group of $n$-th order, Proc. Japan Acad. 42 (1965) 323.

[28] R. V. Kadison, Irreducible Operator Algebras, Proc. Nat. Acad. Sci. U.S.A. 43 (1957) 273.

[29] S. Lang, SL(2, R), Addison-Wesley, Reding etc. 1975.

[30] P. Lax, Functional Analysis, Wiley-Interscience, New York etc. 2002.

[31] J. Lott, Renormalization group flow for general sigma-models, Commun. Math. Phys. 107 (1986) 165.

[32] G. Mack, On Blattner's formula for the discrete series of $\mathrm{SO}_{0}(2 n, 1)$, J. Funct. Anal. 23 (1976) 311.

[33] G. Mackey, Induced Representations, W. A. Benjamin, New York etc., 1968.

[34] K. Maurin, General Eigenfunction Expansions and Unitary Representations of Topological Groups, Polish Scientific Publishers, Warsaw 1968.

[35] P. Michor, Isometric actions of Lie groups and invariants, Lecture notes, Univ. Vienna, 1997, www.mat.univie.ac.at/ michor/tgbook.pdf

[36] N. Mukunda and B. Radhakrishnan, Clebsch-Gordon problem and coefficients of the 3dimensional Lorentz group in a continuous basis, I-IV, J. Math. Phys. 15 (1974) 1320, $1332,1643,1656$. 
[37] H. Narnhofer and W. Thirring, Spontaneously broken symmetries, Ann. Inst. Henri Poincaré, 70 (1999) 1.

[38] M. Niedermaier and E. Seiler, Nonamenability and spontaneous symmetry breaking - the hyperbolic spin chain, Annales Henri Poincaré 6 (2005) 1025-1090 hep-th/0312293.

[39] M. Niedermaier and E. Seiler, Generalized ground states as a limit of the iterated transfer operator, unpublished notes, 2005.

[40] U. Ottoson, A classification of the unitary irreducible representations of $\mathrm{SO}_{0}(1, N)$, Commun. Math. Phys. 8 (1968) 228.

[41] R. Parthasarathy, Dirac operator and discrete series, Ann. Math. 96 (1972) 1-30.

[42] A. Paterson, Amenability, American Mathematical Society, Providence, R.I. 1988.

[43] M. Reed and B. Simon, Methods of Modern Mathematical Physics, vol. 1, revised edition, Academic Press, New York and London 1980.

[44] M. Reed and B. Simon, Methods of Modern Mathematical Physics, vol. 4, Academic Press, New York and London 1978.

[45] D. Ruelle, Statistical mechanics, W.A. Benjamin, 1968.

[46] W. Schmid, Discrete Series, Proc. Symposia in Pure Mathematics 61 (1997) 83-113.

[47] F. Schwarz, Unitary irreducible representations of $\mathrm{SO}_{0}(1, N)$, J. Math. Phys. 12 (1971) 131.

[48] G. L. Sewell, Quantum Mechanics and its Emergent Macrophysics, Princeton University Press, Princeton, N.J. 2002.

[49] T. Spencer and M. Zirnbauer, Spontaneous symmetry breaking of a hyperbolic sigmamodel in three dimensions, Commun. Math. Phys. 252 (2004) 167; math-ph/0410032.

[50] R. Strichartz, Harmonic analysis on hyperboloids, J. Funct. Anal. 12 (1973) 341.

[51] F. Strocchi, Symmetry Breaking, Springer, Berlin etc, 2005.

[52] M. Sugiura, Unitary representations and harmonic analysis, Kodansha Scientific Books, Tokyo 1975.

[53] M. Taylor, Noncommutative harmonic analysis, American Mathematical Society, Providence, R.I. 1986.

[54] E. van den Ban, M. Flensted-Jensen, and H. Schichtkrull, Basic harmonic analysis on pseudo-riemannian symmetric spaces, in: E. Tanner and R. Wilson (Eds), Noncompact Lie groups and some of their applications, p.61-101, Kluwer, Dordrecht 1994.

[55] V. S. Varadarajan, An Introduction to Harmonic Analysis on Semisimple Lie Groups, Cambridge University Press, Cambridge 1989. 
[56] N. Vilenkin and A. Klimyk, Representations of Lie groups and special functions, Vol.2, Kluwer, Dordrecht 1993.

[57] N. Wallach, Harmonic analysis on homogeneous spaces, Marcel Dekker, New York, 1973.

[58] G. Warner, Harmonic Analysis on semisimple Lie groups, Springer-Verlag, Berlin etc. 1972.

[59] S. Weinberg, The Quantum Theory of Fields, vol. I, Cambridge University Press, Cambridge 1995.

[60] R. Zimmer, Ergodic theory and semi-simple groups, Birkhäuser, Boston etc. 1984.

[61] M. Zirnbauer, Migdal-Kadanoff fixed point in the graded nonlinear sigma-model for disordered single particle systems without time reversal symmetry, Phys. Rev. Lett. 60 (1988) 1450 .

[62] M. Zirnbauer, Fourier analysis on a hyperbolic supermanifold with constant curvature, Commun. Math. Phys. 141 (1991) 503.

[63] M. Zirnbauer, Riemannian symmetric spaces and their origin in random matrix theory, J. Math. Phys. 37 (1996) 4986. 